\title{
A Robust Fit for Generalized Additive Models
}

by

\author{
Azadeh Alimadad
}

A thesis submitted to

the Faculty of Graduate Studies and Research

in partial fulfilment of

the requirements for the degree of

Master of Science

School of Mathematics and Statistics

Ottawa-Carleton Institute for Mathematics and Statistics

Carleton University

Ottawa, Ontario, Canada

September, 2004

(C)copyright 2004

Azadeh Alimadad 


$\begin{array}{ll}\begin{array}{l}\text { Library and } \\ \text { Archives Canada }\end{array} & \begin{array}{l}\text { Bibliothèque et } \\ \text { Archives Canada }\end{array} \\ \begin{array}{l}\text { Published Heritage } \\ \text { Branch }\end{array} & \begin{array}{l}\text { Direction du } \\ \text { Patrimoine de l'édition }\end{array} \\ \begin{array}{l}\text { 395 Wellington Street } \\ \text { Ottawa ON K1A ON4 } \\ \text { Canada }\end{array} & \begin{array}{l}\text { 395, rue Wellington } \\ \text { Ottawa ON K1A ON4 } \\ \text { Canada }\end{array}\end{array}$

Your file Votre référence ISBN: 0-494-00773-7

Our file Notre référence

ISBN: 0-494-00773-7

NOTICE:

The author has granted a nonexclusive license allowing Library and Archives Canada to reproduce, publish, archive, preserve, conserve, communicate to the public by telecommunication or on the Internet, loan, distribute and sell theses worldwide, for commercial or noncommercial purposes, in microform, paper, electronic and/or any other formats.

The author retains copyright ownership and moral rights in this thesis. Neither the thesis nor substantial extracts from it may be printed or otherwise reproduced without the author's permission.
AVIS:

L'auteur a accordé une licence non exclusive permettant à la Bibliothèque et Archives Canada de reproduire, publier, archiver, sauvegarder, conserver, transmettre au public par télécommunication ou par l'Internet, prêter, distribuer et vendre des thèses partout dans le monde, à des fins commerciales ou autres, sur support microforme, papier, électronique et/ou autres formats.

L'auteur conserve la propriété du droit d'auteur et des droits moraux qui protège cette thèse. $\mathrm{Ni}$ la thèse ni des extraits substantiels de celle-ci ne doivent être imprimés ou autrement reproduits sans son autorisation.
In compliance with the Canadian

Privacy Act some supporting forms may have been removed from this thesis.

While these forms may be included in the document page count, their removal does not represent any loss of content from the thesis.
Conformément à la loi canadienne sur la protection de la vie privée, quelques formulaires secondaires ont été enlevés de cette thèse.

Bien que ces formulaires aient inclus dans la pagination, il n'y aura aucun contenu manquant. 


\section{Abstract}

Description of the relation between two or more qualitative or quantitative variables is one of the major concern of a statistician, but it is also of interest to other researchers specially in medical sciences. This thesis studies a robust model for generalized additive models for development of some aspects regarding the detection of multiple outliers.

In this thesis we introduce a new fit for GAM models. This fit is more resistant to outliers than the one proposed by Hastie and Tibshirani. This method is based on their General Local Scoring Algorithm, we use this algorithm to fit robust GAM models but instead of using the weights and transformed variables obtained from the maximum likelihood equations for GLM models, we use the weights and transformed variables derived from robust estimating equations for GLM models [3]. 
To:

My grandmother for her wish

My parents for their love

and

My husband Vahid for his support 


\section{Acknowledgements}

I would like to thank my supervisor, Dr. Matías Salibián-Barrera, for his constant and patient assistance and advice throughout the preparation of this thesis, and for all his help and support during the last two years.

Also I would like to thank the School of Mathematics and Statistics, Carleton University and the Department of Epidemiology and Community Medicine, University of Ottawa for providing necessary research facilities for this thesis. Many thanks go to members and staff of these Departments, who helped me during my studies. 


\section{Contents}

Abstract $\quad$ ii

Acknowledgements iii

Contents $\quad$ iv

List of Tables vii

1 Introduction 1

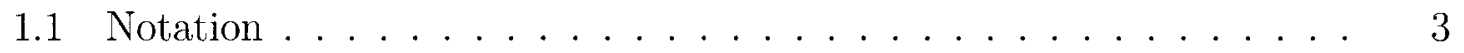

2 Generalized Linear and Additive Models 4

2.1 Generalized Linear Models . . . . . . . . . . . . . . . . . 4

2.2 Maximum Likelihood Estimation . . . . . . . . . . . . . . 9

2.3 Justification of the fitting procedure . . . . . . . . . . . . . 10

2.4 Quasi-Likelihood functions .................. 14

2.5 Additive models . . . . . . . . . . . . . . 16

2.6 Generalized Additive models . . . . . . . . . . . . . . . 18

3 Robust Estimates $\quad 20$

iv 
3.1 Robust Regression .................... . . 20

3.2 Robust GLM and Robust GAM . . . . . . . . . . . . . . . . 27

4 A Robust Fit for GAM

4.1 Introduction . . . . . . . . . . . . . . . . . 31

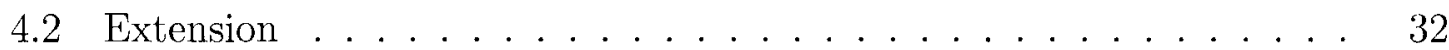

4.2 .1 Simpler approach . . . . . . . . . . . . . . . 32

4.2.2 Approach based on unbiased estimating equations . . . . . . 33

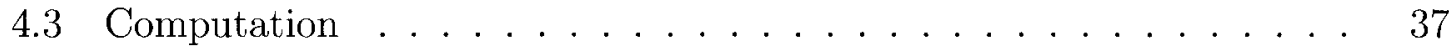

5 Simulation study $\quad 40$

5.1 Calibration of the smoothness parameter . . . . . . . . . . 41

5.2 Outliers in the sample . . . . . . . . . . . . . . 45

$\begin{array}{llr}6 & \text { Example } & 60\end{array}$

$\begin{array}{lll}7 \text { Appendix } & 71\end{array}$ 


\section{List of Tables}

1: Result of simulation to find comparable of span in both methods .

2: Comparison of true outliers and false alarm when span=0.5 and moderate outliers are located in center of the range of the covariate .

3: Comparison of true outliers and false alarm when span $=0.5$ and extreme outliers are located in center of the range of the covariate . .

4: Comparison of true outliers and false alarm when span=0.5 and moderate outliers are located in end of the range of the covariate. . .

5: Comparison of true outliers and false alarm when span=0.5 and extreme outliers are located in end of the range of the covariate . . . 51

6: Comparison of true outliers and false alarm when span $=0.25$ and moderate outliers are located in center of the range of the covariate .

7: Comparison of true outliers and false alarm when span $=0.25$ and extreme outliers are located in center of the range of the covariate .

8: Comparison of true outliers and false alarm when span $=0.25$ and moderate outliers are located in end of the range of the covariate. .

9: Comparison of true outliers and false alarm when span $=0.25$ and extreme outliers are located in end of the range of the covariate . . 
10 : A subset of data which contains weekly new cases of hepatitis C from 8 separate health regions in British Columbia for 262 weeks. . . 


\section{Chapter 1}

\section{Introduction}

Additive Models are a generalization of the linear regression model. The central idea is to replace the linear function of the covariates with the sum of unspecified smooth functions. This model is nonparametric in that we do not specify a parametric form for the functions. Generalized additive models (GAM) are an extension of the class of generalized linear models (GLM). They extend generalized linear models in the same manner that additive models extend the linear regression model. GLM is a powerful and popular technique for modelling a large variety of data. In particular, generalized linear models allow one to model the relationship between the transformed mean response and the covariates for continuous and discrete response variables. However, there is presently a widespread awareness of the dangers posed by the occurrence of outliers, which may be a result of typing errors, misplaced decimal points, recording or transmission errors, members of a different population slipping into the sample, exceptional phenomena and others.

Outliers occur frequently in real data, and they sometimes go unnoticed because usually data is processed by computers, without careful inspection or screening. Ad- 
ditionally, outliers in data with more than 2 variables can be very difficult to detect [25]. Not only the response variable can be outlying, but also the explanatory part, leading to so-called high leverage points. Both types of outliers may severely damage classical analyses. Often, such influential points remain hidden to the user, because they do not always show up in the classical diagnostic plots. To solve this problem, new statistical techniques have been developed that are not so easily affected by outliers. These are called robust (or resistant) methods. Over the past few decades, many researchers studied topics such as robustness and outliers on linear regression models. The fitting algorithm for GAM (General Local Scoring Algorithm) may also be highly sensitive to observations that depart from the model.

In some applications the main interest of the analysis is in detecting potential outliers in the data. For example, if we are trying to detect outbreaks we would like to have a fit that is capable of identifying atypical observations. A consequence of a fit that is affected by the outliers is that it may fail to detect them (as it is effectively trying to accommodate them, and hence they will tend to have a relatively small residual). Moreover, the shift in the fit may cause other ("good") observations to appear as deviating significantly from the model. Resistant methods for GLM, have been introduced by Hampel [7], Pregibon [21], Stefanski, Carroll and Ruppert [27], Künsch, Stefanski and Carroll [14], Bianco and Yohai [2] and Cantoni and Ronchetti [3] among others.

This thesis is organized as follows: The first two chapters consist of introductory and background material. We briefly review generalized linear models, an introduction to Quasi likelihood models, additive models and generalized additive models. Chapter 3 provides Robust regression, Robust generalized linear models and Robust generalized additive models. Chapter 4 presents our proposed Robust GAM, 
discusses the algorithm and some computational details. A simulation study is discussed in Chapter 5 when outliers are present in the data and when the data do not have atypical observation, Chapter 6 contains a real life example that illustrates potential applications of this new method, the appendix contains an implementation in S-PLUS of the algorithms presented in this thesis.

\subsection{Notation}

Here we provide an explanation of some of the notation used in this Thesis. We represent random variables in uppercase, for example $Y_{i}$ and observed values in lowercase, for example $y_{i}$. A vector of random variables is written in boldface as $\mathbf{X}=\left(X_{1}, \ldots, X_{p}\right)$. We use (column) vector notation to represent a sequence of values, such as $\mathbf{x}=\left(x_{1}, \ldots, x_{n}\right)^{t}$ and the $\mathbf{x}_{j}$ the $n$ observed values for variable $j$. A linear predictor refers to an expression of the form $\sum_{j=1}^{p} \mathbf{x}_{i j} \boldsymbol{\beta}_{j}$ and an additive predictor $\alpha+\sum_{j=1}^{p} f_{j}\left(X_{j}\right)$, where $f_{j}\left(X_{j}\right)$ denotes an arbitrary function of variable $X_{j}$. Expectation is denoted by $E$ and variance by var. A log-likelihood is denoted by $l(\theta, \phi ; y)$ or $l$ for short. 


\section{Chapter 2}

\section{Generalized Linear and Additive}

\section{Models}

\subsection{Generalized Linear Models}

The purpose of regression analysis is to use explanatory variables to explain the mean of a response variable of interest. We consider a class of statistical models that is a natural generalization of classical linear models. The classical linear model assumes a relationship of the type

$$
y_{i}=x_{i 1} \beta_{1}+\ldots+x_{i p} \beta_{p}+\varepsilon_{i} \quad \text { for } i=1, \ldots, n
$$

where $n$ is the number of cases, the variables $x_{i 1}, \ldots, x_{i p}$ are called explanatory variables and the variable $y_{i}$ is called the response variable. The random errors $\varepsilon_{i}$ are usually assumed to have a normal probability distribution with mean zero and variance $\sigma^{2}$ and are independent of the explanatory variables. Note that these assumptions about the random errors are necessary for inference on the true regression coefficients 
$\beta_{j}$.

Classical linear models and least squares began with the work of Gauss and Legendre [19], [28]. Let $y_{1}, \ldots, y_{n}$ denote $n$ independent observations. We treat $y_{i}$ as a realization of a random variable $Y_{i}$. In the linear model we assume that $Y_{i}$ has expected value $\mu_{i}$ which is a linear function of $p$ predictors that take values $\mathbf{x}_{i}^{t}=\left(x_{i 1}, \cdots, x_{i p}\right)$ for the i-th case, so that

$$
E\left(Y_{i} \mid \mathbf{x}_{i}\right)=\mu_{i}=\sum_{j=1}^{p} \mathbf{x}_{i j} \boldsymbol{\beta}_{j} \quad i=1 \ldots n,
$$

where the $\beta$ s are parameters whose values are usually unknown and have to be estimated from the data. For many data analysis problems, estimates of the linear relationships between variables are adequate to describe the observed data, and to make reasonable predictions for new observations. However, there are many relationships that cannot adequately be summarized by model (2.1). We will describe some major reasons as follows:

- It may not be reasonable to assume that data are normally distributed, for example the dependent variable of interest may have a non-continuous distribution. For example, suppose we are trying to predict people's family planning choices, specifically how many children families will have, as a function of income and various other socioeconomic indicators. The dependent variable "number of children" is discrete, and most likely the distribution of that variable is highly skewed (i.e., most families have 1,2 , or 3 children, fewer will have 4 or 5 , very few will have 6 or 7 , and so on). In this case it could be reasonable to assume that the dependent variable follows a Poisson distribution.

- The effect of the predictors on the dependent variable may not be linear in 
nature. Thus, the relationship between a person's age and various indicators of health is most likely not linear in nature.

- If the mean of the response variable is naturally restricted to a range of values, the traditional linear model may not be appropriate, since the linear predictor $\mathbf{x}_{i}^{t} \boldsymbol{\beta}$ can take on any value. For example, the mean of a measured proportion is between 0 and 1 , but the linear predictor of the mean in a traditional linear model is not restricted to this range.

The term Generalized Linear Model refers to a larger class of Linear Models [17]. Generalized linear models are an extension of the linear model to include response variables that follow probability distributions in the exponential family of distributions. Generalized Linear Models do not require normality of the response variable, nor do they require homogeneity of variances. Hence Generalized Linear Models can be used when response variables follow distributions other than the normal distribution, and when variances are not constant. For example some count data could be appropriately analyzed as a Poisson random variable within the context of the Generalized Linear Model. For example, we could use GLM to predict the relationship between the number of children in families and some various socioeconomic indicators in the above examples.

We will describe the generalized linear models and discuss the estimation of the parameters. Let $Y_{1}, \ldots, Y_{n}$ be independent random variables with a distribution in the exponential family

$$
f_{Y}\left(y_{i} ; \theta_{i}, \phi\right)=\exp \left\{\frac{\left(y_{i} \theta_{i}-b\left(\theta_{i}\right)\right)}{\phi}+c\left(y_{i}, \phi\right)\right\}
$$

where $\phi, b\left(\theta_{i}\right)$ and $c\left(y_{i}, \phi\right)$ are known functions, $\theta_{i}$ is called the canonical parameter and $\phi$ is called dispersion parameter. The exponential family includes, among others, 
the Normal, Binomial, Poisson, Gamma and Negative Binomial distributions. For example, consider the normal distribution with mean $\mu_{i}$ and variance $\sigma_{i}^{2}$ has density

$$
f\left(y_{i}\right)=\frac{1}{\sqrt{2 \pi \sigma^{2}}} \exp \left\{-\frac{1}{2} \frac{\left(y_{i}-\mu_{i}\right)^{2}}{\sigma^{2}}\right\}
$$

Expanding the square in the exponent we get $\left(y_{i}-\mu_{i}\right)^{2}=y_{i}^{2}+\mu_{i}^{2}-2 y_{i} \mu_{i}$, thus the coefficient of $y_{i}$ is $\mu_{i} / \sigma^{2}$. This result identifies $\theta_{i}$ as $\mu_{i}$ and $\phi$ as $\sigma^{2}$. Therefore

$$
f\left(y_{i}\right)=\exp \left\{\frac{y_{i} \mu_{i}-\frac{1}{2} \mu_{i}^{2}}{\sigma^{2}}-\frac{y_{i}^{2}}{2 \sigma^{2}}-\frac{1}{2} \log \left(2 \pi \sigma^{2}\right)\right\}
$$

this shows that $b\left(\theta_{i}\right)=\frac{1}{2} \theta_{i}^{2}$.

We will now show that if $Y_{i}$ has a density function from (2.3) then the mean and variance are

$$
E\left(Y_{i}\right)=b^{\prime}\left(\theta_{i}\right)
$$

and

$$
\operatorname{var}\left(Y_{i}\right)=b^{\prime \prime}\left(\theta_{i}\right) \phi
$$

To show this we can use general properties of the log-likelihood of distributions in the exponential family as follows.

In general we write $l(\theta, \phi ; y)=\log (L(\theta, \phi ; y))$ for the log-likelihood function considered as a function of $\theta$ and $\phi, y$ being given. The mean and variance of $Y$ can be computed as follows. Let

$$
L(\theta, \phi ; y)=\exp \left\{\sum_{i=1}^{n} \frac{\left(y_{i} \theta_{i}-b\left(\theta_{i}\right)\right)}{\phi}+c\left(\phi, y_{i}\right)\right\}
$$

be the likelihood of the observations $y_{1}, \ldots, y_{n}$. Then their log-likelihood in canonical form is given by

$$
l=\left\{\sum_{i=1}^{n} \frac{\left(y_{i} \theta_{i}-b\left(\theta_{i}\right)\right)}{\phi}+c\right\}
$$


The first derivative is

$$
\frac{\partial l}{\partial \theta_{i}}=\frac{y_{i}-b^{\prime}\left(\theta_{i}\right)}{\phi}
$$

and the second derivative is

$$
\frac{\partial^{2} l}{\partial \theta_{i}^{2}}=\frac{-b^{\prime \prime}\left(\theta_{i}\right)}{\phi}
$$

Since for any distribution in an exponential family [15]

$$
E\left(\frac{\partial l}{\partial \theta_{i}}\right)=0
$$

and

$$
E\left(\frac{\partial^{2} l}{\partial \theta_{i}^{2}}\right)+E\left(\frac{\partial l}{\partial \theta_{i}}\right)^{2}=0,
$$

it immediately follows that $E\left(Y_{i}\right)=b^{\prime}\left(\theta_{i}\right)=\mu_{i}$. Hence

$$
\frac{-b^{\prime \prime}\left(\theta_{i}\right)}{\phi}+\frac{\operatorname{var}\left(Y_{i}\right)}{\phi^{2}}=0 .
$$

Therefore $\operatorname{Var}\left(Y_{i}\right)=b^{\prime \prime}\left(\theta_{i}\right) \phi$. Because $\mu_{i}$ depends on $\theta_{i}$ but not $\phi$, we may write the variance as

$$
\operatorname{Var}\left(Y_{i}\right)=\phi V\left(\mu_{i}\right)
$$

where $V($.$) is called the variance function. The next element of the generalization is$ that instead of directly modelling the mean, as in linear model, we will introduce a one-to one differentiable transformation $g\left(\mu_{i}\right)$ and focus on $g\left(\mu_{i}\right)$. The function $g\left(\mu_{i}\right)$ is called the link function. Examples of link functions include the identity, log and $\operatorname{logit}$, which is defined as $\operatorname{logit}(\mu)=\log \{\mu /(1-\mu)\}$. We further assume that the transformed mean is expressed as a linear model, so that

$$
\eta_{i}=g\left(\mu_{i}\right)=\mathbf{x}_{i}^{t} \boldsymbol{\beta}, \quad i=1,2, \ldots, n .
$$

where $\beta \in \mathbb{R}^{p}$ is vector parameters, $\mathbf{x}_{i} \in \mathbb{R}^{p}$ and the quantity $\eta_{i}$ is called the linear predictor, here are some common examples. Assume that $Y_{i} \sim N\left(\mu_{i}, \sigma^{2}\right)$ and $\mu_{i}=\mathbf{x}_{i}^{t} \boldsymbol{\beta}$ 
in this model. Then $g\left(\mu_{i}\right)=\mu_{i}$ which is the identity link. If $Y_{i} \sim \operatorname{Poisson}\left(\mu_{i}\right)$ and $\mu_{i}=e^{\mathbf{x}_{i}^{t} \boldsymbol{\beta}}$ then, the link function is $g\left(\mu_{i}\right)=\log \left(\mu_{i}\right)$.

\subsection{Maximum Likelihood Estimation}

An important practical feature of generalized linear models is that the maximum likelihood estimate for $\boldsymbol{\beta}$ can be computed using the same algorithm for all distributions in the exponential family [17]. In this section we describe the algorithm as follows:

Given an initial estimate of the parameters $\hat{\boldsymbol{\beta}}_{0}$, we can calculate the estimated linear predictor $\hat{\eta}_{i_{0}}=\mathbf{x}_{i}^{t} \hat{\boldsymbol{\beta}}_{0}$ and use it to obtain the fitted values $\hat{\mu}_{i_{0}}=g^{-1}\left(\hat{\eta}_{i_{0}}\right)$. Using these quantities, we calculate the dependent variables $z_{i_{0}}$ where

$$
z_{i_{0}}=\hat{\eta}_{i_{0}}+\left(y_{i}-\hat{\mu}_{i_{0}}\right) \frac{\partial \eta_{i}}{\partial \mu_{i}}\left(\hat{\boldsymbol{\beta}}_{0}\right), \quad i=1, \ldots, n
$$

Next, we calculate the iterative weights

$$
W_{i_{0}}^{-1}=\left(\frac{\partial \eta_{i}}{\partial \mu_{i}}\right)^{2} V_{i_{0}}
$$

where $V_{i_{0}}$ is the variance function evaluated at $\hat{\mu}_{i_{0}}$. Finally, we obtain an improved estimate of $\boldsymbol{\beta}$ by regressing the dependent variables $z_{i_{0}}$ on the covariates $\mathbf{x}_{i}$ using the weights $W_{i_{0}}$, i.e. we calculate the weighted least-squares estimate

$$
\hat{\boldsymbol{\beta}}_{1}=\left(\mathbf{X}^{t} \mathbf{W}_{\mathbf{0}} \mathbf{X}\right)^{-1} \mathbf{X}^{t} \mathbf{W}_{0} \mathbf{Z}_{\mathbf{0}}
$$

where $\mathbf{W}_{\mathbf{0}}$ is a diagonal matrix of weights with entries $W_{i_{0}}$ given by $(2.7), Z_{0}=$ $\left(z_{1_{0}}, \cdots, z_{n_{0}}\right)$ and $\mathbf{X}$ is the model matrix, 


$$
X=\left(\begin{array}{ccc}
\cdots & \mathrm{x}_{1} & \cdots \\
\cdots & \mathrm{x}_{2} & \cdots \\
& \vdots & \\
\cdots & \mathrm{x}_{n} & \cdots
\end{array}\right)
$$

then we can use $\hat{\boldsymbol{\beta}}_{1}$ to obtain $\hat{\eta}_{i_{1}}$ and by using the appropriate link function we can compute $\hat{\mu}_{i_{1}}, Z_{i}, W_{i}$ and from these we can estimate the $\hat{\boldsymbol{\beta}}_{2}$. The procedure is repeated until some convergence criteria is met. A convenient feature of this algorithm is that it suggests a simple starting procedure to get the iteration under way. For example we could use the data themselves as the first estimate of the mean, $\hat{\mu}_{i_{0}}=y_{i}$ and $\hat{\eta}_{i_{0}}=g\left(\hat{\mu}_{i_{0}}\right)$ therefore from these we can derive $(d \eta / d \mu)_{0}$ and $V_{0}$. Because our proposal to fit the GAM models robustly is based on a similar algorithm, we review the derivation of this algorithm in detail in the following section.

\subsection{Justification of the fitting procedure}

We first show that the maximum-likelihood equations for $\beta$ can be written in the following way:

$$
\frac{\partial l}{\partial \beta}=\sum_{i=1}^{n} W_{i}\left(y_{i}-\mu_{i}\right) \frac{\partial \eta_{i}}{\partial \mu_{i}} \mathbf{x}_{i},
$$

where $W_{i}$ is defined in equation (2.7) above. The log likelihood in canonical form, is given by

$$
l=\sum_{i=1}^{n} \frac{\left(y_{i} \theta_{i}-b\left(\theta_{i}\right)\right)}{\phi}+c
$$


with $E\left(Y_{i}\right)=b^{\prime}\left(\theta_{i}\right)=\mu_{i}, \operatorname{Var}\left(Y_{i}\right)=b^{\prime \prime}\left(\theta_{i}\right) \phi=V\left(\mu_{i}\right)=V_{i}$ and $\theta_{i}=g\left(\mathbf{x}_{i}^{t} \boldsymbol{\beta}\right)$. Now

$$
\frac{\partial l}{\partial \beta}=\frac{1}{\phi} \sum_{i=1}^{n} y_{i} g^{\prime}\left(\mathbf{x}_{i}^{t} \beta\right) \mathbf{x}_{i}-b^{\prime}\left(g\left(\mathbf{x}_{i}^{t} \beta\right)\right) g^{\prime}\left(\mathbf{x}_{i}^{t} \beta\right) \mathbf{x}_{i}
$$

thus

$$
=\sum_{i=1}^{n} \frac{\left(y_{i}-b^{\prime}\left(\theta_{i}\right)\right)\left(g^{\prime}\left(\mathbf{x}_{i}^{t} \beta\right)\right) \mathbf{x}_{i}}{\phi}
$$

We can compute $g^{\prime}\left(\mathbf{x}_{i}^{t} \beta\right) \mathbf{x}_{i}$ by

$$
\frac{\partial \mu_{i}}{\partial \beta}=b^{\prime \prime}\left(g\left(\mathbf{x}_{i}^{t} \beta\right)\right) g^{\prime}\left(\mathbf{x}_{i}^{t} \beta\right) \mathbf{x}_{i}
$$

Therefore,

$$
\frac{\partial l}{\partial \beta}=\sum_{i=1}^{n}\left(y_{i}-b^{\prime}\left(\theta_{i}\right)\right) \frac{\partial \mu_{i}}{\partial \beta} \frac{1}{b^{\prime \prime}\left(g\left(\mathbf{x}_{i}^{t} \beta\right)\right)} \frac{1}{\phi} .
$$

Using the definition of variance implies

$$
\frac{\partial l}{\partial \boldsymbol{\beta}}=\sum_{i=1}^{n} \frac{\left(y_{i}-\mu_{i}\right)}{V_{i}} \frac{\partial \mu_{i}}{\partial \boldsymbol{\beta}} .
$$

Now using the chain rule, we have

$$
\begin{gathered}
\frac{\partial l}{\partial \beta}=\sum_{i=1}^{n} \frac{\left(y_{i}-\mu_{i}\right)}{V_{i}} \frac{\partial \mu_{i}}{\partial \eta_{i}} \frac{\partial \eta_{i}}{\partial \beta} \\
\frac{\partial l}{\partial \beta}=\sum_{i=1}^{n} \frac{\left(y_{i}-\mu_{i}\right)}{V_{i}} \frac{\partial \mu_{i}}{\partial \eta_{i}} \mathbf{x}_{i}
\end{gathered}
$$

and

$$
\frac{\partial l}{\partial \beta}=\sum_{i=1}^{n} \frac{\left(y_{i}-\mu_{i}\right)}{V_{i}}\left(\frac{\partial \mu_{i}}{\partial \eta_{i}}\right)^{-1}\left(\frac{\partial \mu_{i}}{\partial \eta_{i}}\right)^{2} \mathbf{x}_{i}
$$

Hence

$$
\frac{\partial l}{\partial \beta}=\sum_{i=1}^{n} W_{i}\left(y_{i}-\mu_{i}\right) \frac{\partial \eta_{i}}{\partial \mu_{i}} \mathbf{x}_{i}
$$

where

$$
W_{i}^{-1}=V_{i}\left(\frac{\partial \mu_{i}}{\partial \eta_{i}}\right)^{-2}
$$


A procedure that is convenient to solve $\partial l / \partial \boldsymbol{\beta}=\mathbf{0}$ is called dependent variable regression and is a form of iteratively reweighed least squares. This method solves the maximum likelihood equation using Newton-Raphson iterations, which can be derived from a first order Taylor expansion:

$$
\frac{\partial l}{\partial \boldsymbol{\beta}}\left(\boldsymbol{\beta}_{i+1}\right) \approx \frac{\partial l}{\partial \boldsymbol{\beta}}\left(\boldsymbol{\beta}_{i}\right)+\frac{\partial^{2} l}{\partial \boldsymbol{\beta}^{t} \partial \boldsymbol{\beta}}\left(\boldsymbol{\beta}_{i}\right)\left(\boldsymbol{\beta}_{i+1}-\boldsymbol{\beta}_{i}\right)
$$

which suggests the following iterative method:

$$
\boldsymbol{\beta}_{i+1}=\boldsymbol{\beta}_{i}-\left[\frac{\partial^{2} l}{\partial \boldsymbol{\beta}^{t} \partial \boldsymbol{\beta}}\left(\boldsymbol{\beta}_{i}\right)\right]^{-1} \frac{\partial l}{\partial \boldsymbol{\beta}}\left(\boldsymbol{\beta}_{i}\right)
$$

Fisher's scoring method replaces the matrix of second derivatives by its expected value

$$
A=-E\left(\frac{\partial^{2} l}{\partial \boldsymbol{\beta}^{t} \partial \boldsymbol{\beta}}\right)
$$

Given the current estimate $\boldsymbol{\beta}_{i}$ of $\boldsymbol{\beta}$, we derive an adjustment $\Delta \boldsymbol{\beta}_{i}$ as the solution of

$$
\begin{gathered}
\Delta \boldsymbol{\beta}_{i}=\left(\boldsymbol{\beta}_{i+1}-\boldsymbol{\beta}_{i}\right)=A^{-1} \frac{\partial l}{\partial \boldsymbol{\beta}}\left(\boldsymbol{\beta}_{i}\right), \\
\Delta \boldsymbol{\beta}_{i}=A^{-1} \frac{\partial l}{\partial \boldsymbol{\beta}}\left(\boldsymbol{\beta}_{i}\right), \\
A \Delta \boldsymbol{\beta}_{i}=\frac{\partial l}{\partial \boldsymbol{\beta}}\left(\boldsymbol{\beta}_{i}\right) .
\end{gathered}
$$

According to equations (2.5) and (2.6)

$$
E\left(\frac{\partial^{2} l}{\partial \theta^{t} \partial \theta}\right)=-E\left(\frac{\partial l}{\partial \theta}\left(\frac{\partial l}{\partial \theta}\right)^{t}\right)
$$

using equation (2.9) we obtain

$$
A=-E\left(\frac{\partial^{2} l}{\partial \boldsymbol{\beta}^{t} \partial \boldsymbol{\beta}}\right)=E\left(\left(\sum_{i=1}^{n}\left(\frac{y_{i}-\mu_{i}}{V_{i}}\right) \frac{\partial \mu_{i}}{\partial \eta_{i}} \mathbf{x}_{i}\right)\left(\sum_{j=1}^{n}\left(\frac{y_{j}-\mu_{j}}{V_{j}}\right) \frac{\partial \mu_{j}}{\partial \eta_{j}} \mathbf{x}_{j}^{t}\right)\right)
$$

which is

$$
=E\left(\sum_{i, j=1}^{n}\left(\frac{y_{i}-\mu_{i}}{V_{i}}\right) \frac{\partial \mu_{i}}{\partial \eta_{i}}\left(\frac{y_{j}-\mu_{j}}{V_{j}}\right) \frac{\partial \mu_{j}}{\partial \eta_{j}} \mathbf{x}_{i} \mathbf{x}_{j}^{t}\right)
$$


Since for any $i \neq j, y_{i}$ and $y_{j}$ are independent we have

$$
E\left(\left(\frac{y_{i}-\mu_{i}}{V_{i}}\right)\left(\frac{y_{j}-\mu_{j}}{V_{j}}\right)\right)=E\left(\frac{y_{i}-\mu_{i}}{V_{i}}\right) E\left(\frac{y_{j}-\mu_{j}}{V_{j}}\right)
$$

which is equal to zero, hence

$$
A=\sum_{i=1}^{n} E\left(\frac{y_{i}-\mu_{i}}{V_{i}}\right)^{2}\left(\frac{\partial \mu_{i}}{\partial \eta_{i}}\right)^{2} \mathbf{x}_{i} \mathbf{x}_{i}^{t}
$$

or equivalently

$$
A=\sum_{i=1}^{n} \frac{1}{V_{i}^{2}} E\left(y_{i}-\mu_{i}\right)^{2}\left(\frac{\partial \mu_{i}}{\partial \eta_{i}}\right)^{2} \mathbf{x}_{i} \mathbf{x}_{i}^{t}=\sum_{i=1}^{n} V_{i}^{-1}\left(\frac{\partial \mu_{i}}{\partial \eta_{i}}\right)^{2} \mathbf{x}_{i} \mathbf{x}_{i}^{t} .
$$

Using the weights $W_{i}$ in (2.7) and the definition of variance we obtain

$$
A=\sum_{i=1}^{n} W_{i} \mathbf{x}_{i} \mathbf{x}_{i}^{t}
$$

Hence

$$
A \boldsymbol{\beta}=\sum_{i=1}^{n} W_{i} \mathbf{x}_{i} \mathbf{x}_{i}^{t} \boldsymbol{\beta}=\sum_{i=1}^{n} W_{i} \mathbf{x}_{i} \eta_{i}
$$

It has been shown that

$$
\frac{\partial l}{\partial \boldsymbol{\beta}}=\sum_{i=1}^{n} W_{i}\left(y_{i}-\mu_{i}\right) \frac{\partial \eta_{i}}{\partial \mu_{i}} \mathbf{x}_{i}
$$

It follows from equation (2.11) that

$$
A\left(\boldsymbol{\beta}_{i+1}-\boldsymbol{\beta}_{i}\right)=\frac{\partial l}{\partial \boldsymbol{\beta}}\left(\boldsymbol{\beta}_{i}\right)
$$

Therefore,

$$
A \boldsymbol{\beta}_{i+1}=A \boldsymbol{\beta}_{i}+\frac{\partial l}{\partial \boldsymbol{\beta}}\left(\boldsymbol{\beta}_{i}\right)=\sum_{i=1}^{n} W_{i} \mathbf{x}_{i}\left(\eta_{i}+\left(y_{i}-\mu_{i}\right) \frac{\partial \eta_{i}}{\partial \mu_{i}}\right),
$$

which is equal to

$$
\left(\sum_{i=1}^{n} W_{i} \mathbf{x}_{i} \mathbf{x}_{i}^{t}\right) \boldsymbol{\beta}_{i+1}=\sum_{i=1}^{n} W_{i} \mathbf{x}_{i} z_{i}
$$


where $z_{i}=\eta_{i}+\left(y_{i}-\mu_{i}\right) \partial \eta_{i} / \partial \mu_{i}$, which has the form of a weighted least-square equation. From this fact, it follows that

$$
\hat{\boldsymbol{\beta}}_{i+1}=\left(\mathbf{X}^{t} \mathbf{W}_{i} \mathbf{X}\right)^{-1} \mathbf{X}^{t} \mathbf{W}_{i} \mathbf{Z}_{i}
$$

where $\mathbf{W}_{i}$ is a diagonal matrix of weights with entries $w_{i}$ given by (2.7) in the diagonal.

\subsection{Quasi-Likelihood functions}

To define a likelihood we have to specify the form of the distribution for the observed data $y$. Then, the likelihood function for $\theta$ given the data $\mathbf{Y}=\left(y_{1}, \ldots, y_{n}\right)$ is the function

$$
L(\theta \mid \mathbf{y})=f(\mathbf{y} ; \theta)
$$

where $\theta$ is a vector of unknown parameters and the likelihood is interpreted as a function of $\theta$. The maximum likelihood estimate of $\theta$ is the value $\hat{\theta}$ which maximizes the likelihood function, thus

$$
L(\theta \mid \mathbf{y}) \leq L(\hat{\theta} \mid \mathbf{y}) \quad \forall \theta \in \Omega
$$

and $\Omega$ denotes the set of possible values for $\theta$. In practice, $\hat{\theta}$ is obtained either by direct maximization of $\log (L)$, or by solving the equations corresponding to first order conditions:

$$
\mathbf{S}(\theta)=\partial \log L / \partial \theta=0
$$

The function $\mathbf{S}(\theta)$ is known as the score function for $\theta$. As we show in the previous section when the likelihood function comes from the exponential family, the score equations have the same formal expression as

$$
\mathbf{S}(\boldsymbol{\beta})=\sum_{i=1}^{n} \frac{\left(y_{i}-\mu_{i}\right)}{V_{i}} \frac{\partial \mu_{i}}{\partial \beta}=0
$$


(see equation 2.8) and the solution $\hat{\boldsymbol{\beta}}$, can be obtained by iterative reweighted least squares-type algorithm (see Section (2.3)).

Often there is no theory available on the random mechanism by which the data were generated. One important property of the GLM family is that the score function, (2.13), depends only on the mean and variance of the response variables $Y_{i}$. Wedderburn [29] was the first to point out that the estimation equation (2.13) can therefore be used to estimate the regression coefficients for any choice of link function, mean and variance functions. He defined quasi-likelihood functions where we only need to specify how the response mean is related to the covariates and how the variance of the observations is related to the mean of the observations. Moreover, he showed that when the variances of observations are known functions and specified to be either equal to, or proportional to, some function of their means, but the distributions are unknown, the regression parameters can be consistently estimated through the use of quasi-likelihoods. The quasi likelihood of an observation for distributions of the exponential family is the same as the log-likelihood.

McCullagh [16] described a wider class of problems, he examined the connection between quasi-likelihood functions, exponential family models and nonlinear weighted least squares. He discussed consistency and asymptotic normality of the parameter estimates under second moment assumptions.

We concentrate mainly on the case in which the observations are independent and where the effect of interest can be described by a model for $E\left(Y_{i}\right)$. Suppose we have independent observations $y_{i}, i=1, \ldots, n$ with the mean $\mu_{i}$ and variance $\operatorname{Var}\left(Y_{i}\right)=$ $\phi V\left(\mu_{i}\right)$, where $V$ is a known function. We suppose that for each observation $\mu_{i}$ is a known function of a set of parameters $\beta_{1}, \ldots, \beta_{p}$. The quasi-likelihood estimator is the solution of the system of estimating equations 


$$
\sum_{i=1}^{n} \frac{\partial}{\partial \boldsymbol{\beta}} Q\left(y_{i}, \mu_{i}\right)=\sum_{i=1}^{n} \frac{\left(y_{i}-\mu_{i}\right)}{V\left(Y_{i}\right)} \frac{\partial \mu_{i}}{\partial \boldsymbol{\beta}},
$$

where the $Q\left(y_{i}, \mu_{i}\right)$ is the quasi-likelihood function. As in ordinary likelihood estimation, we can start with an initial value for $\hat{\boldsymbol{\beta}}_{0}$, then by using the Newton-Raphson and Fisher's scoring method we can iteratively find a sequence of $\hat{\boldsymbol{\beta}}_{i}$ 's until convergence occurs.

\subsection{Additive models}

General non-linear models take the following form,

$$
Y=f\left(X_{1}, \ldots X_{p}\right)+\varepsilon
$$

where $X_{1}, \ldots, X_{p}$ are explanatory variables, $Y$ is the response variable, $\varepsilon$ are random errors and $f: \mathbb{R}^{p} \longrightarrow \mathbb{R}$ is an arbitrary smooth function. The non-linear model becomes difficult to fit in. general for $p>2$. Also it is not easy to interpret general non-linear regression when there are many explanatory variables. An additive model [9] represents a generalization of multiple regression models which is a special case of linear models. It replaces the simple terms of the linear equation $\boldsymbol{\beta}_{j} \mathbf{x}_{j}$ with $f_{j}\left(X_{j}\right)$ where $f_{j}$ is an arbitrary smooth function of the predictor $X_{j}$. In other words, instead of a single coefficient for each explanatory variable, an unspecified function is estimated for each predictor, to achieve a better prediction of the dependent variable values. An additive model with response variable $Y$ and explanatory variables $X_{1}, \cdots, X_{p}$ has the form

$$
Y=\alpha+\sum_{j=1}^{p} f_{j}\left(X_{j}\right)+\varepsilon
$$


where the errors $\varepsilon$ are independent of the $X_{j} \mathrm{~s}, E(\varepsilon)=0$ and $\operatorname{var}(\varepsilon)=\sigma^{2}$. The $f_{j} \mathrm{~s}$ are arbitrary univariate functions, one for each predictor. Implicit in (2.15) is the assumption that $E\left\{f_{j}\left(X_{j}\right)\right\}=0$, otherwise there will be free constants in each of the functions and the intercept $\alpha$ will be unidentifiable.

The term additive means that the model is a sum of terms, an interaction like $X_{1} X_{2}$ may not be incorporated into the model unless they are defined as new predictors $X_{3}=X_{1} X_{2}$. More generally, one may allow terms like $f\left(X_{1}, X_{2}\right)$ where $f(.,$.$) is$ an unspecified function, estimated by a bivariate smoother. Since

$$
E\left(Y \mid X_{1}, \ldots, X_{p}\right)=f\left(X_{1}, \ldots, X_{p}\right)=\sum_{j=1}^{p} f_{j}\left(X_{j}\right),
$$

the effect of $X_{j}$ on $E(Y)$ is the same for any value of the other covariates. We use LOESS (Locally-weighted regression) [5], to estimate the $f_{j}$ s and we will identify them with $S$. We use a procedure called back-fitting [9] to estimate $f_{j}\left(X_{j}\right)$, it is a general algorithm that can fit an additive model using any regression-type fitting mechanisms and it is based on the following observation. If the additive model (2.15) is correct, for any $1 \leq k \leq p$ we have

$$
Y-\alpha-\sum_{j \neq k} f_{j}\left(X_{j}\right)=f_{k}\left(X_{k}\right)+\varepsilon
$$

By assumption $\varepsilon$ and $X_{k}$ are independent so taking conditional expectation implies $E\left(Y-\alpha-\sum_{j \neq k} f_{j}\left(X_{j}\right) \mid X_{k}\right)=E\left(f_{k}\left(X_{k}\right) \mid X_{k}\right)+E\left(\varepsilon \mid X_{k}\right)$. Since $E\left(\varepsilon \mid X_{k}\right)=$ $E(\varepsilon)=0$ and for any measurable function $g$ and random variable $Z, E(g(Z) \mid Z)=$ $g(Z)$, therefore $E\left(Y-\alpha-\sum_{j \neq k} f_{j}\left(X_{j}\right) \mid X_{k}\right)=f_{k}\left(X_{k}\right)$. This immediately suggests an iterative algorithm for computing all the estimates $S_{j}$.

- Initialize:

$$
S_{0}=\bar{Y}, \quad S_{1}^{0}=S_{2}^{0}=\ldots=S_{p}^{0}=0, \quad m=0
$$


- Iterate:

$$
\begin{gathered}
m=m+1, \text { and for } 1 \leq j \leq p \text { do }: \\
R_{j}=Y-S_{0}-\sum_{k \neq j} S_{k}^{(m-1)} \text { and } S_{j}^{(m)}=S\left(R_{j} \mid X_{j}\right),
\end{gathered}
$$

where $S(u \mid z)$ denotes a smoother of $u$ as a function of $z$.

- Repeat until:

$$
R S S^{(m)}=\operatorname{avg}\left(Y-S_{0}^{(m)}-\sum_{j=1}^{p} S_{j}^{(m)}\left(X_{j}\right)\right)^{2} \text { fails to decrease. }
$$

where avg denotes the average over the sample.

The resulting iterative procedure is known as the back-fitting algorithm [6]. A weighted back-fitting algorithm has the same form as above, except that the smoothers are weighted.

\subsection{Generalized Additive models}

Whereas linear models assume that the response is linear in each predictor, additive models assume only that the response is affected by each predictor in a smooth way. Generalized additive models [17] extend linear models and generalized linear models by flexibly modelling additive nonlinear relationships between the predictors and the response. Generalized additive models permit the response probability distribution to be any member of the exponential family of distributions. We assume that the response variable $Y$ has a distribution given by equation (2.3), with the mean $\mu=$ $E\left(Y \mid X_{1}, \ldots, X_{p}\right)$ linked to the predictors via

$$
g(\mu)=S_{0}+\sum_{j=1}^{p} S_{j}\left(X_{j}\right)
$$


Estimation of $S_{0}$ and $S_{1}, \ldots, S_{p}$ is accomplished by replacing the weighted linear regression in the adjusted dependent variable by an appropriate algorithm for fitting a weighted additive model which is called the General Local Scoring Algorithm, the procedure is outlined as follows.

- Initialize:

$$
S_{0}^{0}=g(\bar{Y}), S_{1}^{0}=S_{2}^{0}=\ldots=S_{p}^{0}=0, m=0
$$

- Update: Construct an adjusted dependent variable

$$
z_{i}^{0}=\eta_{i}^{0}+\left(y_{i}-\mu_{i}^{0}\right)\left(\frac{\partial \eta_{i}}{\partial \mu_{i}}\right)
$$

with $\eta_{i}^{0}=S_{0}^{0}+\sum_{j=1}^{p} S_{j}^{0}\left(X_{i j}\right), \mu_{i}^{0}=g^{-1}\left(\eta_{i}^{0}\right)$ and weights $W_{i}^{0}=\left(\partial \mu_{i} / \partial \eta_{i}\right)^{2}\left(V_{i}^{0}\right)^{-1}$. Then we should fit a weighted additive model to $z_{i}$, to obtain estimated functions $S_{j}^{1}$, additive predictors $\eta_{i}^{1}$, and fitted values $\mu_{i}^{1}$ and compute the convergence criterion

$$
\Delta\left(\eta^{1}, \eta^{0}\right)=\frac{\sum_{j=1}^{p}\left\|S_{j}^{1}-S_{j}^{0}\right\|}{\sum_{j=1}^{p}\left\|S_{j}^{0}\right\|}
$$

where $S_{j}$ is a vector of $\mathrm{n}$ real numbers and $\|$.$\| is the Euclidean norm.$

- Repeat replacing $\eta^{m}$ by $\eta^{m+1}$ until $\Delta\left(\eta^{m+1}, \eta^{m}\right)$ is below some small threshold.

This algorithm is borrowed from the iteratively reweighted least squares algorithm for generalized linear models (see Section (2.2)). 


\section{Chapter 3}

\section{Robust Estimates}

\subsection{Robust Regression}

A resistant fit is the one which is not sensitive to large changes in a few observations. Resistant methods are useful in data analysis because they give an alternative way of looking at the data, when such an approach is warranted. In particular, resistant methods can lead to the identification of outlying observations. Points identified as outliers can then be carefully revised to determine what may have caused them. The practitioner can study these observations more carefully to establish their cause. In other words, we use robust methods to detect potentially anomalous observations, but assessing their practical significance is left to the practitioner as sometimes subjectmatter expertise is needed for this.

Consider a classical linear model

$$
y_{i}=x_{i 1} \beta_{1}+\ldots+x_{i p} \beta_{p}+\varepsilon_{i} \text { for } i=1, \ldots, n
$$

where $x_{i}$ are the predictor vectors and $y_{i}$ are the dependent variables. The residual $r_{i}$ of the $i$ th case is the difference between what is actually observed and what is 
estimated, for a given estimate $\tilde{\boldsymbol{\beta}}$ we can obtain $\tilde{y}_{i}=x_{i 1} \tilde{\beta}_{1}+\ldots+x_{i p} \tilde{\beta}_{p}$ therefore $\tilde{r}_{i}=y_{i}-\tilde{y}_{i}$. The most popular linear regression estimator was introduced by Gauss and Legendre [19] and [28]. It corresponds to,

$$
\hat{\boldsymbol{\beta}}=\min _{\tilde{\boldsymbol{\beta}}} \sum_{i=1}^{n} \tilde{r}_{i}^{2} .
$$

The basic idea is to optimize the fit by making the average of the squared residuals small, this is the well-known Least squares method. Since real data sometimes present atypical observations, consider an example to see the effect of outliers in the simple regression model with $p=2$,

$$
y_{i}=\beta_{0}+\beta_{1} x_{i}+\varepsilon_{i}
$$

where the slope $\beta_{1}$ and the intercept $\beta_{0}$ are to be estimated. The scatterplot of $\left(x_{1}, y_{1}\right), \ldots,\left(x_{15}, y_{15}\right)$ is shown as follows.

Therefore, the least square solution fits the data very well, as can be seen from the least squares line $\hat{y}=\hat{\beta}_{0}+\hat{\beta}_{1} x$ in the plot. Now, suppose that someone gets a wrong value of $y_{2}$ then $\left(x_{2}, y_{2}\right)$ may be rather far away from the "ideal" line. This point is called an outtier in the $y$-direction and it has large influence on the new least squares line, which is quite different from the least squares line above. 


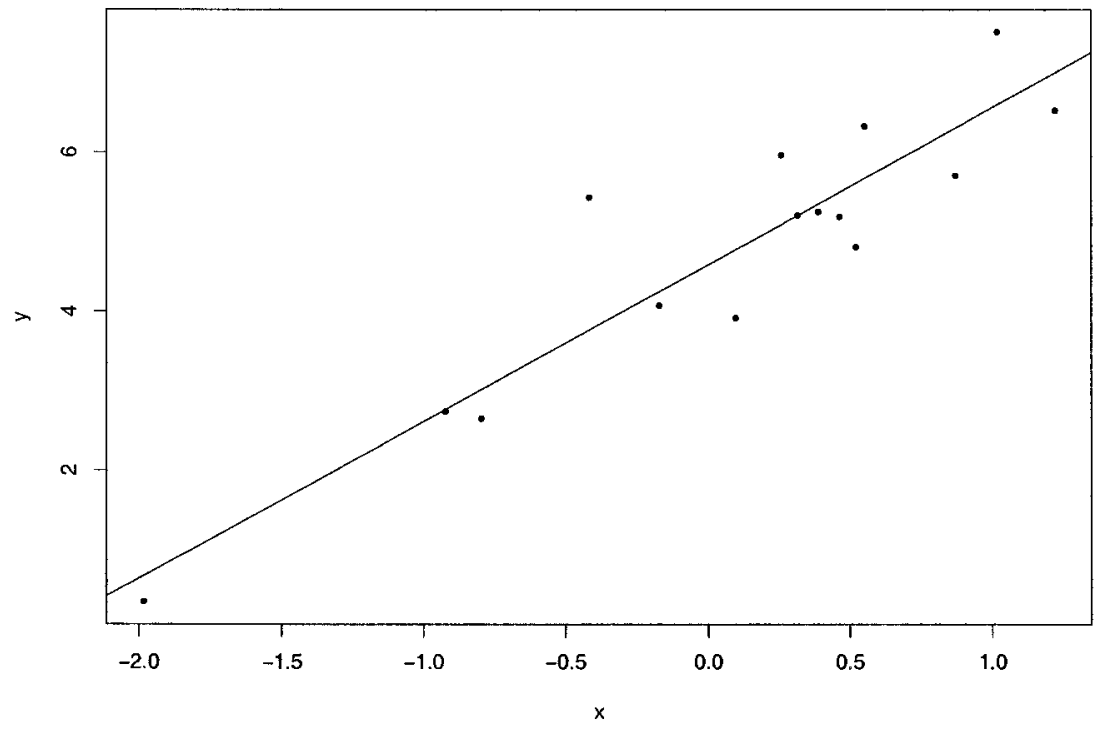

Figure 3.1: ThIS GRAPH SHOWS ALL THE 15 RANDOM POINTS. 


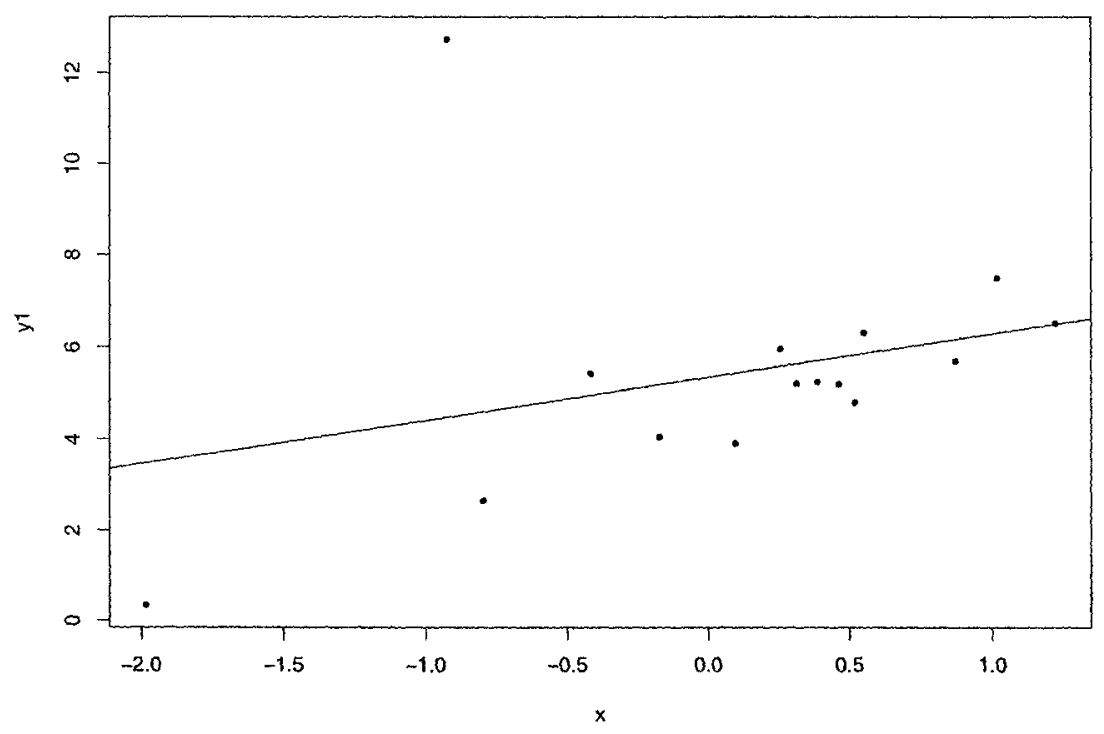

Figure 3.2: THIS GRAPH SHOWS AN OUTLIER IN $y$-DIRECTION IN THE DATA. 


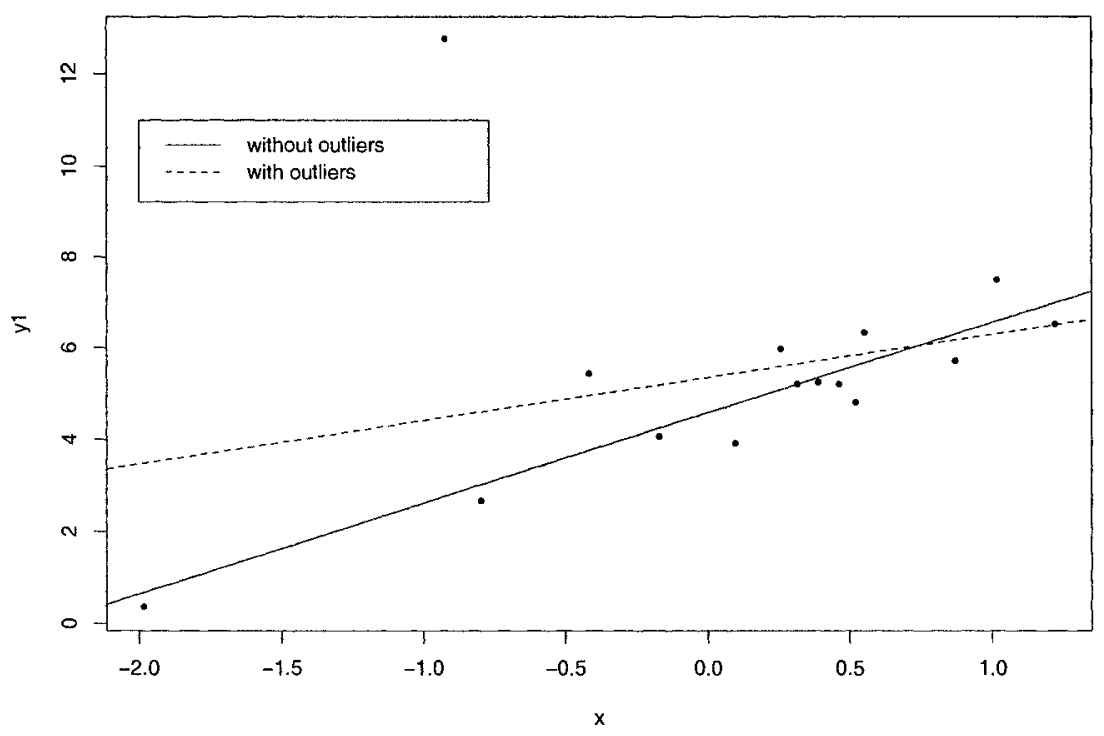

Figure 3.3: THIS GRAPH SHOWS BOTH LEAST SQUARE FITS.

Also there may be atypical values on the explanatory variables. These will be more difficult to detect when there are several explanatory variables (since we can not plot them). Observations that have explanatory variables far from the bulk of the data are generally highly influential (high-leverage) and can seriously affect the LS fit. To illustrate the effect of a high-leverage observation in the example, suppose that the 3rd observation $x_{3}$ was wrongly recorded, we obtain 


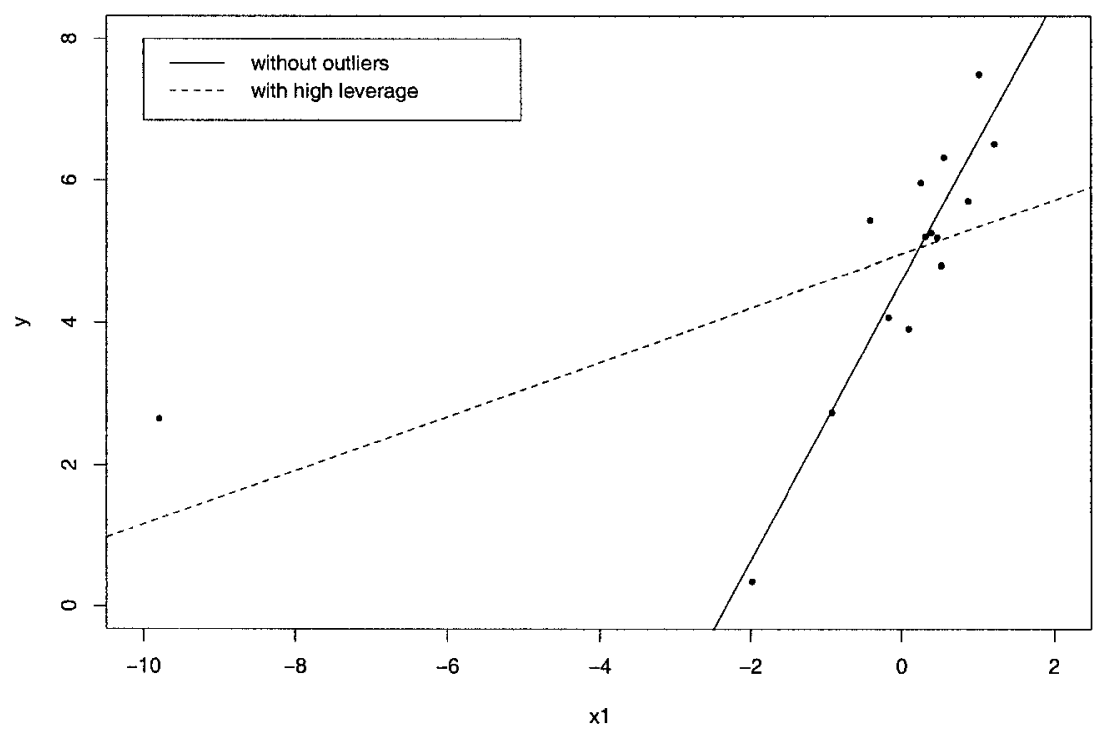

Figure 3.4: THIS GRAPH SHOWS BOTH LEAST SQUARE FITS.

the resulting point is called an outlier in the $x$-direction and its effect on the least squares estimator is very large because it actually tilts the least square line. Therefore the point $\left(x_{3}, y_{3}\right)$ above, is called a high leverage point. In general, we call an observation $\left(x_{k}, y_{k}\right)$ a high leverage point whenever $x_{k}$ lies far away from the bulk of the observed $x_{i}$ in the sample and are highly influential on regression coefficient.

One approach to deal with outliers is called regression diagnostics. Diagnostics are certain quantities computed from the data with the purpose of identify precisely influential points, after which these outliers can be removed or corrected, followed by an least square analyis on the remaining cases. Scveral diagnostic methods have been designed to detect individual cases or groups of cases that may differ from the most of the data (see, for example, [18]). Many diagnostics are based on the residuals resulting from least square. However, this starting point may lead to useless result 
because of the following reason.

- By definition, least square tries to avoid large residuals. Therefore one outlying case may cause a poor fit for the majority of the data. Because the least square estimator tries to accommodate this case at the expense of the remaining observations.

- Masking: when there is more than one outlier in the data, they may be very difficult to identify using most diagnostic and outlier detection tools (see, for example, [22], [25]).

As an illustration, consider Figure (3.4) the residuals $r_{1}$ and $r_{2}$ are much larger than the residual $r_{3}=y_{3}-\hat{y_{3}}$, although they correspond to "good" points. If one would apply a rule like "delete" the points with largest LS residuals, then the "good" points would have to be deleted first.

Another approach is robust regression which tries to devise estimators so that they are not strongly affected by outliers. Robust regression analysis aims at identifying potential outliers so that they can be studied further. This usually can not be done by means of the least square residuals. Therefore, diagnostics and robust regression really have the same goals, only in the opposite order: When using diagnostics tools, one first tries to delete the outliers and then to fit the "good" data by least squares, whereas a robust analysis first wants to fit a regression to the majority of the data and then to discover the outliers as those points which possess large residuals from that robust solution.

One popular robust technique is called $M$-estimators [10], [11], [12], [13]. Let $r_{i}$ denote the difference between the observation and its fitted value (residual). The standard least-squares method tries to minimize $\sum_{i} r_{i}^{2}$, which is unstable if there are 
outliers present in the data. But the $M$-estimators try to reduce the effect of outliers by replacing the squared residuals by another loss function $\rho($.$) so that$

$$
\hat{\boldsymbol{\beta}}=\min _{\boldsymbol{\beta}} \sum_{i=1}^{n} \rho\left(r_{i}\right)
$$

where $\rho$ is a symmetric, positive function with a unique minimum at zero, and is typically chosen to increase slower than $u^{2}$ for large values of $|u|$. For example, for the least squares $\rho(u)=u^{2}$ or for Huber function

$$
\rho_{k}(u)= \begin{cases}u^{2} / 2 & |u| \leq k \\ k(|u|-k / 2) & |u|>k\end{cases}
$$

where $k$ is a positive fixed constant. Various other estimators have been proposed such as least median of squares (LMS) estimator [23], least trimmed squares (LTS) [23], $S$-estimators [24], MM-estimators [30] and $\tau$-estimators [31].

\subsection{Robust GLM and Robust GAM}

There have been some proposals in the literature to extend robust estimators to GLM models. Pregibon [20] introduced a loss function which is less sensitive to influential data points than the log-likelihood function. Consider the deviance function

$$
d(p ; y)=2 l_{\max }(\theta ; y)-2 l(\theta ; y)
$$

where $l_{\max }(\theta ; y)$ is the maximum of $l(\theta ; y)$ with respect to $\theta$. Since $l_{\max }(\theta ; y)$ does not depend on the parameters, it is constant with respect to $\theta$. Therefore maximizing $l(\theta ; y)$ is equivalent to minimizing $d(p ; y)$. A modification of maximum likelihood (minimum deviance) estimation is simply to replace $d(p ; y)$ by a function which is less sensitive to observations poorly accounted for by the model. 
Pregibon [21] defined robust estimates by modifying the ML-estimate which is obtained by maximizing the log likelihood

$$
\sum_{i=1}^{n}\left[Y_{i} \ln \left(P\left(\mathbf{x}_{i}^{t} \boldsymbol{\beta}\right)\right)+\left(1-Y_{i}\right) \ln \left(1-P\left(\mathbf{x}_{i}^{t} \boldsymbol{\beta}\right)\right)\right],
$$

or its equivalent to minimizing $D(\boldsymbol{\beta} ; y)=\sum_{i=1}^{n} D_{i}\left(\boldsymbol{\beta} ; y_{i}\right)$ where $D_{i}\left(\boldsymbol{\beta} ; y_{i}\right)$ are the deviances. Since, ML-estimate is extremely sensitive to the presence of unusual data in the sample, Pregibon proposed to minimize

$$
\sum_{i=1}^{n} \rho\left(D_{i}\left(\boldsymbol{\beta} ; y_{i}\right)\right)
$$

where $\rho$ is a Huber type function in the family given by

$$
\rho(t)= \begin{cases}t & t \leq c \\ 2(t c)^{1 / 2}-c & t>c\end{cases}
$$

Unfortunately the estimators defined by the minimization of (3.2) are not consistent.

Bianco and Yohai [2], proposed a class of $M$-estimators for logistic regression models which can be thought as a Fisher-consistent version of the estimators given by (3.2). These estimators are defined by the minimization of

$$
\sum_{i=1}^{n}\left[\rho\left(D_{i}(\boldsymbol{\beta}) ; y_{i}\right)+G\left(P\left(\mathbf{x}_{i}^{\prime} \boldsymbol{\beta}\right)\right)+G\left(1-P\left(\mathbf{x}_{i}^{\prime} \boldsymbol{\beta}\right)\right)\right],
$$

where $\rho$ is a bounded, differentiable and nondecreasing function and

$$
G(t)=\int_{0}^{t} \psi(-\ln u) d u
$$

where $\psi(t)=\rho^{\prime}(t)$. The fact that $\rho$ is bounded makes these estimates qualitatively robust. The estimates defined by the minimization of above equations are a natural generalization of the $M$-estimates with bounded $\rho$ function used for regression, which is resistant and satisfy consistency. 
Recently Cantoni and Ronchetti [3] proposed robust estimators for generalized linear models based on quasi-likelihood. They consider a general class of $M$-estimators as the solution of the estimating equations,

$$
\sum_{i=1}^{n} \phi\left(y_{i}, \mu_{i}\right)=0
$$

where

$$
\phi(y, \mu)=v(y, \mu) \frac{\partial \mu}{\partial \boldsymbol{\beta}}-a(\boldsymbol{\beta}) .
$$

and $a(\boldsymbol{\beta})$ is a correction factor, to ensure that the above estimating equations are unbiased, that is

$$
a(\boldsymbol{\beta})=\frac{1}{n} \sum_{i=1}^{n} E\left[v\left(y_{i}, \mu_{i}\right)\right] \frac{\partial \mu_{i}}{\partial \boldsymbol{\beta}},
$$

Note that if $v(y, \mu)=\left(y_{i}-\mu_{i}\right) / v_{i}$ then $a(\boldsymbol{\beta})=0$ and we obtain the classical quasi likelihood equation. To obtain robust estimates Cantoni and Ronchetti proposed to use

$$
v\left(y_{i}, \mu_{i}\right)=\psi_{c}\left(r_{i}\right) \frac{1}{\sqrt{v_{i}}}
$$

where $r_{i}=\left(y_{i}-\mu_{i}\right) / \sqrt{v_{i}}$ are the Pearson residuals and $\psi_{c}$ is the Huber function defined by

$$
\psi_{c}(r)= \begin{cases}r & |r| \leq c \\ c \operatorname{sign}(\mathrm{r}) & |r|>c\end{cases}
$$

Therefore, the estimating equations are

$$
\sum_{i=1}^{n} \psi_{c}\left(\frac{y_{i}-\mu_{i}}{\sqrt{v_{i}}}\right) \frac{1}{\sqrt{v_{i}}} \frac{\partial \mu_{i}}{\partial \boldsymbol{\beta}}-a(\boldsymbol{\beta})=\mathbf{0} .
$$

The solutions $\hat{\boldsymbol{\beta}}_{n}$ of the equation above are asymptotically normal

$$
\sqrt{n}\left(\hat{\boldsymbol{\beta}}_{n}-\boldsymbol{\beta}\right) \longrightarrow N(0, \Sigma)
$$


and they have good robustness properties [3].

Bianco and Boente [1] proposed robust estimates for generalized nonparametric models which obtain consistency and asymptotic normality result. Hastie and Tibshirani [8] consider likelihood and partial likelihood-based estimates but they note that for a general score function the local scoring procedure can still be used.

In the next chapter we extend these ideas to introduce a new robust fit for GAM models. 


\section{Chapter 4}

\section{A Robust Fit for GAM}

\subsection{Introduction}

As in the case of generalized linear models, outliers can heavily influence fitting generalized additive models. In some applications it is of interest to detect those observations that deviate from the rest. Hence it is of interest to develop robust fits for generalized additive models.

Assume that $Y_{1}, \ldots, Y_{n}$ are independent random variables with a distribution in the exponential family

$$
f_{Y}\left(y_{i} ; \theta_{i}, \phi\right)=\exp \left\{\frac{\left(y_{i} \theta_{i}-b\left(\theta_{i}\right)\right)}{\phi}+c\left(y_{i}, \phi\right)\right\},
$$

with mean $\mu=E\left(Y \mid X_{1}, \ldots, X_{p}\right)$ linked to the predictors via

$$
g(\mu)=S_{0}+\sum_{j=1}^{p} S_{j}\left(X_{j}\right),
$$

where $g($.$) is the link function. The main idea of our proposal is to use the General$ Local Scoring Algorithm (GLSA) described in Chapter 2 using weights derived from 
robust estimating equations for GLM. More specifically, we use robust quasi score functions

$$
\sum_{i=1}^{n} \psi_{c}\left(\frac{y_{i}-\mu_{i}}{\sqrt{v_{i}}}\right) \frac{1}{\sqrt{v_{i}}} \frac{\partial \mu_{i}}{\partial \boldsymbol{\beta}}-a(\boldsymbol{\beta})=\mathbf{0}
$$

where $\psi_{c}(u)$ is the Huber function (3.6) and $a(\boldsymbol{\beta})$ is a correction factor (3.5). Note that the effect of $\psi_{c}$ is to downweight observations with large residuals. In the following sections we develop this idea in more detail.

\subsection{Extension}

\subsubsection{Simpler approach}

Consider the robust quasi likelihood equations of [3] and [27]. A first approach is to ignore the bias-correction term $a(\boldsymbol{\beta})$, hence in this case the robust quasi score function changes to

$$
\sum_{i=1}^{n} \psi_{c}\left(\frac{y_{i}-\mu_{i}}{\sqrt{v_{i}}}\right) \frac{1}{\sqrt{v_{i}}} \frac{\partial \mu_{i}}{\partial \boldsymbol{\beta}}=\mathbf{0}
$$

Although we expect the estimators based on (4.2) to be biased in general, the derivation of our proposal is easier to follow in this case. In section 4.2 .2 we develop the general method based on (4.1).

By multiplying and dividing by $\left(y_{i}-\mu_{i}\right) / \sqrt{v_{i}}$ we will get the equivalent following equation

$$
\sum_{i=1}^{n} \frac{\left(y_{i}-\mu_{i}\right)}{\sqrt{v_{i}}} \frac{\sqrt{v_{i}}}{\left(y_{i}-\mu_{i}\right)} \psi_{c}\left(\frac{y_{i}-\mu_{i}}{\sqrt{v_{i}}}\right) \frac{1}{\sqrt{v_{i}}} \frac{\partial \mu_{i}}{\partial \boldsymbol{\beta}}=\mathbf{0} .
$$

We can use the same derivation that applies to generalized linear models, thus we will have 


$$
\sum_{i=1}^{n} \psi_{c}\left(\frac{y_{i}-\mu_{i}}{\sqrt{v_{i}}}\right) \frac{1}{\sqrt{v_{i}}} \frac{\partial \mu_{i}}{\partial \boldsymbol{\beta}}=\sum_{i=1}^{n}\left(y_{i}-\mu_{i}\right) \frac{\partial \eta_{i}}{\partial \mu_{i}} A_{i} W_{i} \mathbf{x}_{i}
$$

where

$$
W_{i}=\left(\frac{\partial \mu_{i}}{\partial \eta_{i}}\right)^{2} V_{i}^{-1}
$$

and

$$
A_{i}=\frac{\psi_{c}\left(\frac{y_{i}-\mu_{i}}{\sqrt{v_{i}}}\right)}{\frac{y_{i}-\mu_{i}}{\sqrt{v_{i}}}}=\frac{\psi_{c}\left(U_{i}\right)}{U_{i}} .
$$

Comparing the above equation with the same equation in generalized linear models, immediately suggests that we can use the reweighted least square algorithm with weights

$$
\tilde{W}_{i}=W_{i} A_{i}
$$

to solve the equation (4.2). Since these robust but biased estimators can be found using re-weighted least squares with weights $\tilde{W}_{i}$, then to fit the corresponding GAM model we can use the General Local Scoring Algorithm with weights $\tilde{W}_{i}$ as well.

\subsubsection{Approach based on unbiased estimating equations}

Since in (4.2) we are ignoring the correction term $a(\boldsymbol{\beta})$ the estimating equations are not unbiased. In general, for estimating equations, consistency follows from unbiasedness of the equations, i.e. an estimate $\hat{\theta}$ defined as the solution of

$$
\sum_{i=1}^{n} H\left(x_{i}, \hat{\theta}\right)=0
$$

will satisfy $\hat{\theta} \longrightarrow \theta_{0}$ if $E_{\theta_{0}}\left\{H\left(x, \theta_{0}\right)\right\}=0$. Hence we require that equation (4.2) be unbiased, i.e.

$$
E\left(\sum_{i=1}^{n} \psi_{c}\left(\frac{y_{i}-\mu_{i}}{\sqrt{v_{i}}}\right) \frac{1}{\sqrt{v_{i}}} \frac{\partial \mu_{i}}{\partial \boldsymbol{\beta}}-a(\beta)\right)=0
$$


If we use the unbiased equations, we need to solve $f(\boldsymbol{\beta})=\mathbf{0}$ where

$$
f(\boldsymbol{\beta})=\sum_{i=1}^{n}\left[\left(\psi_{c}\left(r_{i}\right) \frac{1}{\sqrt{v_{i}}} \frac{\partial \mu_{i}}{\partial \eta_{i}} \mathbf{x}_{i}-a(\boldsymbol{\beta})\right]\right.
$$

or

$$
\sum_{i=1}^{n}\left(\psi_{c}\left(r_{i}\right) \frac{1}{\sqrt{v_{i}}} \frac{\partial \mu_{i}}{\partial \eta_{i}} \mathbf{x}_{i}\right)-n(a(\boldsymbol{\beta}))=\mathbf{0}
$$

Note that

$$
a(\boldsymbol{\beta})=\frac{1}{n} \sum_{i=1}^{n} E\left(\psi_{c}\left(r_{i}\right)\right) \frac{1}{\sqrt{v_{i}}} \frac{\partial \mu_{i}}{\partial \eta_{i}} \mathbf{x}_{i} .
$$

Hence the equation to be solved is

$$
f(\boldsymbol{\beta})=\sum_{i=1}^{n}\left[\left(\psi_{c}\left(r_{i}\right) \frac{1}{\sqrt{v_{i}}} \frac{\partial \mu_{i}}{\partial \eta_{i}} \mathbf{x}_{i}-n\left[\frac{1}{n} \sum_{i=1}^{n} E\left(\psi_{c}\left(r_{i}\right)\right) \frac{1}{\sqrt{v_{i}}} \frac{\partial \mu_{i}}{\partial \eta_{i}} \mathbf{x}_{i}\right]=\mathbf{0}\right.\right.
$$

or equivalently

$$
f(\boldsymbol{\beta})=\sum_{i=1}^{n}\left[\psi_{c}\left(r_{i}\right)-E\left(\psi_{c}\left(r_{i}\right)\right)\right] \frac{1}{\sqrt{v_{i}}} \frac{\partial \mu_{i}}{\partial \eta_{i}} \mathbf{x}_{i}=\mathbf{0} .
$$

A one-step Taylor expansion suggests the following iterations:

$$
f\left(\boldsymbol{\beta}_{i+1}\right)=f\left(\boldsymbol{\beta}_{i}\right)+\left(\nabla f\left(\boldsymbol{\beta}_{i}\right)\right)\left(\boldsymbol{\beta}_{i+1}-\boldsymbol{\beta}_{i}\right)=\mathbf{0},
$$

where $\nabla f$ denotes the gradient of $f$. Therefore

$$
\left(\nabla f\left(\boldsymbol{\beta}_{i}\right)\right)\left(\boldsymbol{\beta}_{i+1}-\boldsymbol{\beta}_{i}\right)=-f\left(\boldsymbol{\beta}_{i}\right)
$$

thus

$$
\left(\nabla f\left(\boldsymbol{\beta}_{i}\right)\right) \boldsymbol{\beta}_{i+1}=\left(\nabla f\left(\boldsymbol{\beta}_{i}\right)\right) \boldsymbol{\beta}_{i}-f\left(\boldsymbol{\beta}_{i}\right),
$$

by getting the expectation from the equation above, we will have

$$
E\left(\nabla f\left(\boldsymbol{\beta}_{i}\right)\right) \boldsymbol{\beta}_{i+1}=E\left(\left(\nabla f\left(\boldsymbol{\beta}_{i}\right)\right) \beta_{i}\right)-f\left(\boldsymbol{\beta}_{i}\right)
$$


In equation (4.3) consider

$$
h_{i}=\psi_{c}\left(r_{i}\right)-E\left(\psi_{c}\left(r_{i}\right)\right) \text {, }
$$

so the gradient of $f$ is

$$
\nabla f\left(\boldsymbol{\beta}_{i}\right)=\sum_{i=1}^{n}\left\{\frac{\partial h_{i}}{\partial \boldsymbol{\beta}} \frac{1}{\sqrt{v_{i}}}+h_{i} \frac{\partial}{\partial \boldsymbol{\beta}}\left[\left(v_{i}^{1 / 2}\right)\right]\right\} \frac{\partial \mu_{i}}{\partial \eta_{i}} \mathbf{x}_{i} .
$$

Since $E\left(h_{i}\right)=0$, we will have

$$
E\left(\nabla f\left(\boldsymbol{\beta}_{i}\right)\right)=\sum_{i=1}^{n} E\left(\frac{\partial h_{i}}{\partial \beta}\right) \frac{1}{\sqrt{v_{i}}} \frac{\partial \mu_{i}}{\partial \eta_{i}} \mathbf{x}_{i} .
$$

Let $\gamma_{i}=E\left(\partial h_{i} / \partial \boldsymbol{\beta}\right)$. Then

$$
f(\boldsymbol{\beta})=\sum_{i=1}^{n} h_{i} \frac{1}{\sqrt{v_{i}}} \frac{\partial \mu_{i}}{\partial \eta_{i}} \mathbf{x}_{i}
$$

and

$$
E\left(\nabla f\left(\boldsymbol{\beta}_{i}\right) \boldsymbol{\beta}\right)=\sum_{i=1}^{n} \gamma_{i} \frac{1}{\sqrt{v_{i}}} \frac{\partial \mu_{i}}{\partial \eta_{i}} \mathbf{x}_{i}^{t} \boldsymbol{\beta}
$$

also

$$
\frac{\partial h_{i}}{\partial \boldsymbol{\beta}}=\psi_{c}^{\prime}\left(r_{i}\right) \frac{\partial r_{i}}{\partial \boldsymbol{\beta}}-\frac{\partial}{\partial \boldsymbol{\beta}} E\left(\psi_{c}\left(r_{i}\right)\right) .
$$

Since we assume $r_{i}=\left(y_{i}-\mu_{i}\right) / \sqrt{v_{i}}$ we will have

$$
\frac{\partial r_{i}}{\partial \boldsymbol{\beta}}=-\frac{\partial \mu_{i} / \partial \boldsymbol{\beta} \sqrt{v_{i}}-\left(y_{i}-\mu_{i}\right) \partial / \partial \boldsymbol{\beta} \sqrt{v_{i}}}{v_{i}} .
$$

Therefore,

$$
\psi_{c}^{\prime}\left(r_{i}\right) \frac{\partial r_{i}}{\partial \boldsymbol{\beta}}=-\frac{\psi_{c}^{\prime}\left(r_{i}\right)}{\sqrt{v_{i}}} \frac{\partial \mu_{i}}{\partial \boldsymbol{\beta}}-1 / 2 \psi_{c}^{\prime}\left(r_{i}\right) \frac{\left(y_{i}-\mu_{i}\right)}{\sqrt{v_{i}}} \frac{1}{v_{i}} \frac{\partial v_{i}}{\partial \boldsymbol{\beta}}
$$

As we know

$$
\gamma_{i}=E\left(\frac{\partial h_{i}}{\partial \boldsymbol{\beta}}\right)=E\left(\psi_{c}^{\prime}\left(r_{i}\right)\right) \frac{\partial r_{i}}{\partial \boldsymbol{\beta}}-E\left(\frac{\partial}{\partial \boldsymbol{\beta}} E\left(\psi_{c}\left(r_{i}\right)\right)\right)
$$


Using the last 2 equations implies

$$
\gamma_{i}=\left[-\frac{E\left(\psi_{c}^{\prime}\left(r_{i}\right)\right)}{\sqrt{v_{i}}} \frac{\partial \mu_{i}}{\partial \eta_{i}}-1 / 2 E\left(\psi_{c}^{\prime}\left(r_{i}\right) r_{i}\right) \frac{1}{v_{i}} \frac{\partial v_{i}}{\partial \eta_{i}}-E\left(\frac{\partial}{\partial \eta_{i}} E\left(\psi_{c}\left(r_{i}\right)\right)\right)\right] \mathbf{x}_{i}=\ell_{i} \mathbf{x}_{i}
$$

Therefore

$$
E\left(\nabla f\left(\boldsymbol{\beta}_{i}\right) \boldsymbol{\beta}_{i}\right)=\sum_{i=1}^{n} \gamma_{i} \frac{1}{\sqrt{v_{i}}} \frac{\partial \mu_{i}}{\partial \eta_{i}} \mathbf{x}_{i}^{t} \boldsymbol{\beta}_{i}
$$

which is equal to

$$
E\left(\nabla f\left(\boldsymbol{\beta}_{i}\right) \boldsymbol{\beta}_{i}\right)=\sum_{i=1}^{n} \ell_{i} \frac{1}{\sqrt{v_{i}}} \frac{\partial \mu_{i}}{\partial \eta_{i}} \mathbf{x}_{i} \mathbf{x}_{i}^{t} \boldsymbol{\beta}_{i} .
$$

Using equations (4.4) and (4.5)

$$
\begin{gathered}
E\left(\nabla f\left(\boldsymbol{\beta}_{i}\right) \boldsymbol{\beta}_{i}\right)-f(\boldsymbol{\beta})=\sum_{i=1}^{n} \frac{\ell_{i}}{\sqrt{v_{i}}} \frac{\partial \mu_{i}}{\partial \eta_{i}} \mathbf{x}_{i} \eta_{i}-\sum_{i=1}^{n} h_{i} \frac{1}{\sqrt{v_{i}}} \frac{\partial \mu_{i}}{\partial \eta_{i}} \mathbf{x}_{i} \\
=\sum_{i=1}^{n}\left(\ell_{i} \eta_{i}-h_{i}\right) \frac{1}{\sqrt{v_{i}}} \frac{\partial \mu_{i}}{\partial \eta_{i}} \mathbf{x}_{i}
\end{gathered}
$$

therefore

$$
=\sum_{i=1}^{n} \ell_{i} \frac{1}{\sqrt{v_{i}}} \frac{\partial \mu_{i}}{\partial \eta_{i}}\left(\eta_{i}-\frac{h_{i}}{\ell_{i}}\right) \mathbf{x}_{i}
$$

Finally, our iterations

$$
E\left(\nabla f\left(\boldsymbol{\beta}_{i}\right) \boldsymbol{\beta}_{i+1}\right)=E\left(\nabla f\left(\boldsymbol{\beta}_{i}\right)\right) \beta_{i}-f\left(\boldsymbol{\beta}_{i}\right)
$$

becomes

$$
\left(\sum_{i=1}^{n} \ell_{i} \frac{1}{\sqrt{v_{i}}} \frac{\partial \mu_{i}}{\partial \eta_{i}} \mathbf{x}_{i} \mathbf{x}_{i}^{t}\right) \boldsymbol{\beta}_{i}=\sum_{i=1}^{n} \ell_{i} \frac{1}{\sqrt{v_{i}}} \frac{\partial \mu_{i}}{\partial \eta_{i}}\left(\eta_{i}-\frac{h_{i}}{\ell_{i}}\right) \mathbf{x}_{i} .
$$

Which has the form

$$
\left(\sum_{i=1}^{n} W_{i} \mathbf{x}_{i} \mathbf{x}_{i}^{t}\right) \boldsymbol{\beta}_{i+1}=\sum_{i=1}^{n} W_{i} \mathbf{x}_{i} z_{i}
$$

where

$$
z_{i}=\eta_{i}-\frac{h_{i}}{\ell_{i}}
$$


and

$$
W_{i}=\ell_{i}-\frac{1}{\sqrt{v_{i}}} \frac{\partial \mu_{i}}{\partial \eta_{i}}
$$

that has the form of linear weighted least-squares equation hence:

$$
\hat{\boldsymbol{\beta}}_{i+1}=\left(\mathbf{X}^{t} \mathbf{W}_{i} \mathbf{X}\right)^{-1} \mathbf{X}^{t} \mathbf{W}_{i} \mathbf{Z}_{i}
$$

where $\mathbf{W}_{i}$ is a diagonal matrix of weights with entries $W_{i}$ given by (4.6) in the diagonal. This RWLS representation suggests that we can use the local-scoring algorithm to fit Robust GAM models using the above weights and adjusted responses.

\subsection{Computation}

According to the previous section we can use the following algorithm to obtain a robust fit for GAM.

1. Initialize:

$$
S_{0}^{0}=g\left(\operatorname{ave}\left(y_{i}\right)\right), \quad S_{1}^{0}=S_{2}^{0}=\ldots=S_{P}^{0}=0, \quad m=0
$$

2. Update: Construct an adjusted dependent variable

$$
z_{i}^{0}=\eta_{i}-\frac{h_{i}}{\ell_{i}}
$$

and weights

$$
W_{i}=\ell_{i} \frac{1}{\sqrt{v_{i}}} \frac{\partial \mu_{i}}{\partial \eta_{i}}
$$

we fit a weighted back-fitting algorithm to $z_{i}$, to obtain estimated functions $S_{j}^{1}$, additive predictor $\eta^{1}$, and and compute the convergence criterion

$$
\Delta\left(\eta^{1}, \eta^{0}\right)=\frac{\sum_{j=1}^{p}\left\|S_{j}^{1}-S_{j}^{0}\right\|}{\sum_{j=1}^{p}\left\|S_{j}^{0}\right\|}
$$


3. Repeat $\operatorname{step}(2)$ replacing $\eta^{m}$ by $\eta^{m+1}$ until $\Delta\left(\eta^{m+1}, \eta^{m}\right)$ is below some small threshold.

Therefore we need to show how to compute the weights $W_{i}$ and adjusted dependent variables $z_{i}$. We showed

$$
z_{i}=\eta_{i}-\frac{h_{i}}{\ell_{i}}
$$

where $\eta_{i}=S+\sum_{j=1}^{p} S_{j}\left(X_{i j}\right), h_{i}=\psi_{c}\left(r_{i}\right)-E\left(\psi_{c}\left(r_{i}\right)\right)$ and

$$
\ell_{i}=-\frac{E\left(\psi^{\prime}\left(r_{i}\right)\right)}{\sqrt{v_{i}}} \frac{\partial \mu_{i}}{\partial \eta_{i}}-1 / 2 E\left(\psi^{\prime}\left(r_{i}\right) r_{i}\right) \frac{1}{v_{i}} \frac{\partial v_{i}}{\partial \eta_{i}}-E\left(\frac{\partial}{\partial \eta_{i}} E\left(\psi\left(r_{i}\right)\right)\right)
$$

hence we need to show how we can compute $E\left(\psi_{c}\left(r_{i}\right)\right), E\left(\psi_{c}\left(r_{i}\right)\right), E\left(\psi_{c}^{\prime}\left(r_{i}\right) r_{i}\right)$ and $E\left(\frac{\partial}{\partial \eta_{i}} E\left(\psi_{c}\left(r_{i}\right)\right)\right)$ when $r_{i}$ is a Pearson residuals and $\psi$ is Huber's function

$$
\psi_{c}(r)= \begin{cases}r & |r| \leq c \\ c \operatorname{sign}(\mathrm{r}) & |r|>c\end{cases}
$$

Now we show how to compute the expected values that are required for $h_{i}$ and $\ell_{i}$. First consider

$$
E\left(\psi_{c}\left(r_{i}\right)\right)=E\left[\psi_{c}\left(\frac{y_{i}-\mu_{i}}{\sqrt{v_{i}}}\right)\right]
$$

since this function is constant outside [-c, c], hence we will define $j_{1}=\mu_{i}-c V^{1 / 2}$ and $j_{2}=\mu_{i}+c V^{1 / 2}$. For all $Y_{i} \sim P\left(\mu_{i}\right)$ so that $E\left[Y_{i}\right]=V\left(\mu_{i}\right)=\mu_{i}$. Then we will have

$$
E\left[\psi_{c}\left(\frac{y_{i}-\mu_{i}}{\sqrt{v_{i}}}\right)\right]=\sum_{j=0}^{+\infty} \psi_{c}\left(\frac{j-\mu_{i}}{\sqrt{v_{i}}}\right) P\left(Y_{i}=j\right)
$$

which is equal to

$$
=c\left[P\left(Y_{i} \geq j_{2}+1\right)-P\left(Y_{i} \leq j_{1}\right)\right]+\frac{\mu_{i}}{\sqrt{v_{i}}}\left[P\left(Y_{i}=j_{1}\right)-P\left(Y_{i}=j_{2}\right)\right] .
$$

Then

$$
E\left(\psi_{c}^{\prime}\left(r_{i}\right)\right)=E\left[\psi_{c}^{\prime}\left(\frac{y_{i}-\mu_{i}}{\sqrt{v_{i}}}\right)\right]
$$


we have

$$
\psi_{c}^{\prime}(r)= \begin{cases}1 & |r| \leq c \\ 0 & |r|>c\end{cases}
$$

and hence

$$
E\left[\psi_{c}^{\prime}\left(\frac{y_{i}-\mu_{i}}{\sqrt{v_{i}}}\right)\right]=\sum_{j=0}^{+\infty} \psi_{c}^{\prime}\left(\frac{j-\mu_{i}}{\sqrt{v_{i}}}\right) P\left(Y_{i}=j\right)
$$

or

$$
=P\left(Y_{i} \leq j_{2}\right)-P\left(Y_{i}<j_{1}\right)
$$

Now consider

$$
E\left(\psi_{c}^{\prime}\left(r_{i}\right) r_{i}\right)=E\left[\psi_{c}^{\prime}\left(\frac{y_{i}-\mu_{i}}{\sqrt{v_{i}}}\right) \frac{y_{i}-\mu_{i}}{\sqrt{v_{i}}}\right]
$$

so we have

$$
\begin{gathered}
E\left[\psi_{c}^{\prime}\left(\frac{y_{i}-\mu_{i}}{\sqrt{v_{i}}}\right)\left(\frac{y_{i}-\mu_{i}}{\sqrt{v_{i}}}\right)\right]=\sum_{j=0}^{+\infty} \psi_{c}^{\prime}\left(\frac{j-\mu_{i}}{\sqrt{v_{i}}}\right)\left(\frac{j-\mu_{i}}{\sqrt{v_{i}}}\right) P\left(Y_{i}=j\right) \\
=\sum_{j=j_{1}}^{j_{2}} \frac{j-\mu_{i}}{\sqrt{v_{i}}} P\left(Y_{i}=j\right),
\end{gathered}
$$

and finally,

$$
E\left(\frac{\partial}{\partial \eta_{i}} E\left(\psi_{c}\left(r_{i}\right)\right)\right)
$$

to solve this equation we can use the numerical derivative

$$
\frac{\partial}{\partial \eta_{i}} E\left(\psi_{c}\left(r_{i}\right)\right)=\lim \frac{\Delta E\left(\psi_{c}\left(r_{i}\left(\eta_{i}\right)\right)\right)}{\Delta \eta_{i}}
$$

when $\Delta \eta_{i} \longrightarrow 0$.

We pick a small value of $\Delta \eta_{i}=2 h$ (e.g., 10E-5) to approximate the left-hand side by the value of the right-hand side, which is the difference of $E\left(\psi_{c}\left(r_{i}\left(\eta_{i_{0}}\right)\right)\right)$ and $E\left(\psi_{c}\left(r_{i}\left(\eta_{i_{1}}\right)\right)\right)$ respect to variation of $\Delta \eta_{i}$. Where $r_{i}\left(\eta_{i_{0}}\right)=\left(y_{i}-\mu_{i_{0}}\right) / \sqrt{v_{i_{0}}}, \eta_{i_{0}}=\eta-h$ and $\eta_{i_{1}}=\eta+h$. 


\section{Chapter 5}

\section{Simulation study}

In this chapter we present the results of a simulation study designed to compare the performance of the robust fit for GAM models discussed in Chapter 4 with Hastie and Tibshirani's estimation method as implemented in S-PLUS. In particular we compared our method with the function gam in S-PLUS 6. This function allows two estimation methods for the non-linear predictors: loess and splines. We focused on loess estimates. Moreover, although not well documented, the function gam can produce a robust fit by using the option family=robust ().

We implemented our adaptation of the Local Scoring Algorithm in S-PLUS. As a result the smoothing parameter of the loess part of the estimation in our code and in that of S-PLUS may not be comparable. Hence we ran a small simulation to calibrate this parameter (i.e. to find which values of it produced similar results when the data followed the specified model). This study is discussed in Section 5.1. We call the S-PLUS fit obtained with the function gam and the option family=robust $S$-PLUS robust fit, the fit obtained with the robust algorithm of Chapter 4 will be called RGAM fit. 
In Section 5.2 we present the results of our simulation comparing the behavior of the S-PLUS robust fit with the robust fit for GAM models discussed in Chapter 4. We concentrated on the case of responses following Poisson distributions.

\subsection{Calibration of the smoothness parameter}

Because we want to make a fair comparison between the S-PLUS robust fit and the RGAM fit proposed in this Thesis, we conducted a simulation study to determine which bandwidth values are equivalent for the different algorithms. The bandwidth used in the smoothing part of both these algorithms is determined by the argument span. This is a fixed value $\alpha$ between 0 and 1 that determines the proportion of data points that will be included in each window. Specifically, if there are $n$ points in the data, then the $[n \alpha]$ nearest neighbours of each observation are included in each window, where $[u]$ denotes the integer part of $u$. We used $\alpha=0.25$ and $\alpha=$ 0.50 for RGAM. The following simulation was designed to determine the equivalent bandwidths in S-PLUS's gam function when there are no outliers in the data. We used a non-linear model with one covariate in 5000 random samples, where each sample contained 200 observations where $\left(Y_{i} \mid X_{i}\right) \sim P\left(\mu_{i}\right)$. We chose $\mu_{i}=\exp (-\cos (7 *$ $\left.\left.\mathbf{x}_{i} / 120\right) / 1.2+1 / 1.2\right)$. This function is clearly non-linear, and has 2 full cycles in the range $0 \leq \mathbf{x}_{i} \leq 200$. The shape of this function will later allow us to add outliers in several different interesting locations. Hence we will be able to explore the effect of atypical data points in various positions relative to the rest of the data.

For each of the 5,000 samples we used the following formula to compare the difference between the true and predicted mean in both methods:

$$
\sum_{i=1}^{n}\left(\frac{\hat{\mu}_{i}-\mu_{i}}{\sqrt{\mu_{i}}}\right)^{2},
$$


where $\hat{\mu}_{i}$ is the fitted value for the $i$-th observation. Our estimates are as in section 4.2 with function $\psi_{k}$ as in equation (3.6). We used $K=0.5,1.0$, and 1.5 with span= 0.25 , and 0.5 for our model and compared the results of the robust fit of Chapter 4 and that of gam for various values of the smoothness parameter span $(0.05,0.10$, $0.175,0.20,0.225,0.25$ and 0.30$)$. In each case we generated 5,000 samples following the above model. The results are shown in the following Table. 


\begin{tabular}{|c|c|c|c|}
\hline \multicolumn{5}{|c|}{ S-PLUS robust fit } \\
\hline Span & K=0.5 & K=1 & K=1.5 \\
& mean (sd) & mean (sd) & mean (sd) \\
\hline 0.05 & $30.68(8.36)$ & $30.92(7.61)$ & $30.77(7.17)$ \\
0.10 & $16.40(6.16)$ & $16.34(5.56)$ & $15.92(5.16)$ \\
0.15 & $12.83(5.69)$ & $12.16(4.99)$ & $11.41(4.53)$ \\
0.175 & $12.45(5.73)$ & $11.45(4.96)$ & $10.52(4.41)$ \\
0.20 & $12.87(6.02)$ & $11.20(5.11)$ & $9.91(4.48)$ \\
0.225 & $13.68(6.31)$ & $11.42(5.30)$ & $9.83(4.6)$ \\
0.25 & $15.80(6.90)$ & $12.50(5.71)$ & $10.34(4.92)$ \\
0.30 & $22.18(8.24)$ & $16.47(6.64)$ & $13.05(5.78)$ \\
\hline \multicolumn{4}{|c|}{ RGAM fit } \\
\hline Span & K=0.5 & K=1 & K=1.5 \\
& mean (sd) & mean (sd) & mean (sd) \\
\hline 0.25 & $14.58(5.88)$ & $12.86(5.15)$ & $12.14(4.83)$ \\
0.50 & $11.24(5.12)$ & $10.38(4.61)$ & $10.01(4.38)$ \\
\hline
\end{tabular}

Table 1: Mean and standard deviation of the loss FUnCtion (5.1) for 5000 random samples, without outLiers, TO EVALUATE WHICH SPAN IN S-PLUS ROBUST FIT IS EQUIVALENT TO SPANS OF 0.25 AND 0.5 IN RGAM FIT FOR DIFFERENT VALUE OF THE TUNING CONSTANT K

This table shows that when we use the $\operatorname{span}=0.5$ and $\operatorname{span}=0.25$ in RGAM fit we will get very similar fits in S-PLUS robust fit, using the $\mathrm{span}=0.2$ and $\mathrm{span}=0.15$ respectively. We used the above information to illustrate both fits, the solid line corresponds to RGAM fit when $\mathrm{span}=0.5$ and the dash line demonstrate the S-PLUS robust fit with span of 0.2 . 


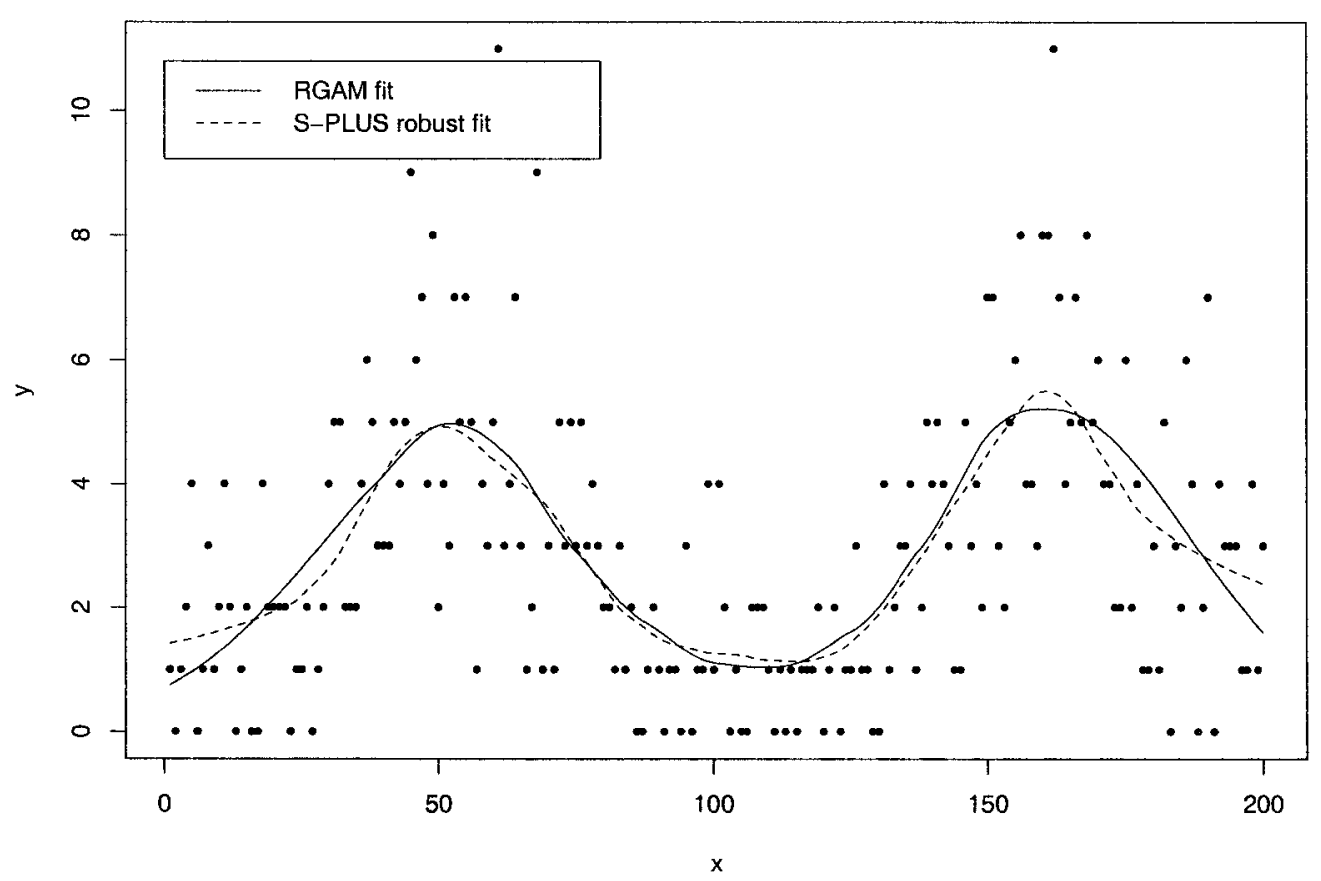

Figure 5.1: THIS GRAPH SHOWS RGAM FIT WITH SPAN $=0.5$ AND S-PLUS ROBUST FIT WITH SPAN $=0.2$ WHEN THERE ARE NO OUTLIERS IN THE DATA. 


\subsection{Outliers in the sample}

We used the same non-linear model as in the previous section with one covariate in 5000 random samples with span of 0.25 and 0.5 in RGAM fit, also we consider $\mathrm{K}=0.5$, $K=1$ and $K=1.5$ for each span where each sample contained 200 observations. We contaminated the data and put between 5 and $10 \%$ of outliers in our samples. We consider different locations for the outliers, the center and the upper end of the range of the covariate. We also considered different magnitudes for the outliers, moderate (relatively close to the rest of the data) and extreme (farther away from the curve).

For each of the 5000 samples, since we contaminated the data, we can identify the true outliers. Then for each method we obtained predicted means and by using the responses we identified the observation with tail probability less than $\alpha(0.01$, $0.001,0.0005$ and 0.0001), label them as outliers (predicted outliers) and denote the outliers predicted by the RGAM fit and the S-PLUS robust fit by RPO and SPO, respectively. We compared the true outliers with the predicted outliers to check the accuracy of each method in prediction of the true outliers. In each method we consider the difference between the predicted and the true outliers as predicted false outliers.

For each sample we used the following formula to compare the difference between the true and predicted mean in both methods:

$$
\sum_{i=1}^{n}\left(\frac{\hat{\mu}_{i}-\mu_{i}}{\sqrt{\mu_{i}}}\right)^{2},
$$

where $\hat{\mu}_{i}$ is the fitted value for the $i$-th observation. Then we compute the mean and standard deviation of these quantities for all 5000 samples in each methods and denote them in the following tables with M-RGAM fit and M-S-PLUS robust fit. In 
each method for all 5000 samples we compute

$$
\frac{\text { Number of true outliers predicted by each method }}{\text { Number of predicted outliers by each method }} * 100
$$

and

the difference of predicted outliers and true outliers predicted by each method $* 100$. predicted outliers by each method

In the following tables we show the mean and standard deviation of predicted true outliers and predicted false outliers obtained with the RGAM and S-PLUS robust fits. In these tables RPTO and RPFO denote the predicted true and false outliers as obtained with the RGAM fit, respectively. Similarly, SPTO and SPFO denote the predicted true and false outliers that resulted from using the S-PLUS robust fit. 
$\operatorname{span}=0.5$

\begin{tabular}{|c|c|c|c|c|}
\hline $\begin{array}{c}\text { Moderate } \\
\text { Center }\end{array}$ & $\begin{array}{c}\alpha=0.01 \\
\text { mean }(\mathrm{sd})\end{array}$ & $\begin{array}{l}\alpha=0.001 \\
\operatorname{mean}(\mathrm{sd})\end{array}$ & $\begin{array}{l}\alpha=0.0005 \\
\operatorname{mean}(\mathrm{sd})\end{array}$ & $\begin{array}{l}\alpha=0.0001 \\
\operatorname{mean}(\mathrm{sd})\end{array}$ \\
\hline $\mathrm{K}=0.5$ & \multicolumn{2}{|c|}{ M-RGAM fit $=16.45(7.71)$} & \multicolumn{2}{|c|}{ M-S-PLUS robust fit $=32.95(13.23)$} \\
\hline RPTO & $100(0.00)$ & $100(0.00)$ & $100(0.00)$ & $100(0.00)$ \\
\hline SPTO & $100(0.00)$ & $100(0.00)$ & $100(0.00)$ & $100(0.00)$ \\
\hline RPFO & $37.35(1.94)$ & $7.20(1.03)$ & $4.15(0.64)$ & $1.22(0.20)$ \\
\hline SPFO & $33.74(1.97)$ & $5.52(0.83)$ & $3.09(0.47)$ & $0.77(0.12)$ \\
\hline $\mathrm{K}=1$ & \multicolumn{2}{|c|}{ M-RGAM fit=16.11(7.31) } & \multicolumn{2}{|c|}{ M-S-PLUS robust fit $=37.49(13.21)$} \\
\hline RPTO & $100(0.00)$ & $100(0.00)$ & $100(0.00)$ & $100(0.00)$ \\
\hline SPTO & $100(0.00)$ & $100(0.00)$ & $100(0.00)$ & $99.98(0.0039)$ \\
\hline RPFO & $36.45(1.913)$ & $6.83(0.98)$ & $3.91(0.60)$ & $1.13(0.18)$ \\
\hline SPFO & $32.71(1.912)$ & $5.21(0.78)$ & $2.83(0.43)$ & $0.71(0.11)$ \\
\hline $\mathrm{K}=1.5$ & \multicolumn{2}{|c|}{ M-RGAM fit $=17.28(7.63)$} & \multicolumn{2}{|c|}{ M-S-PLUS robust fit $=50.68(16.61)$} \\
\hline RPTO & $100(0.00)$ & $100(0.00)$ & $100(0.00)$ & $100(0.00)$ \\
\hline SPTO & $100(0.00)$ & $100(0.00)$ & $100(0.00)$ & $99.9(0.02)$ \\
\hline RPFO & $35.57(1.86)$ & $6.57(0.94)$ & $3.68(0.57)$ & $1.05(0.17)$ \\
\hline SPFO & $31.06(1.85)$ & $4.61(.69)$ & $2.48(0.38)$ & $0.58(0.09)$ \\
\hline
\end{tabular}

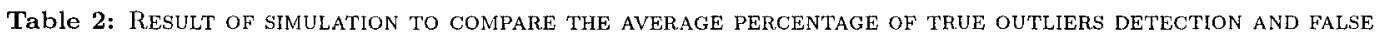
ALARM AS OBTAINED WITH RGAM AND S-PLUS RoBUST GAM FOR DIFFERENT VALUES OF ThE TUNING CONSTANT K AND SPAN $=0.5$, WHEN MODERATE OUTLIERS ARE LOCATED IN CENTER OF THE RANGE OF THE COVARIATE. 
To compare the RGAM fit with the S-PLUS robust fit we use a boxplot to show the shape of the distributions, their central values, and the variability of the fits, using equation (5.1) with 5000 samples for each method. For example, the following graph is referred to the Table 2 where $\mathrm{k}=0.5$. As we can see in this example, two

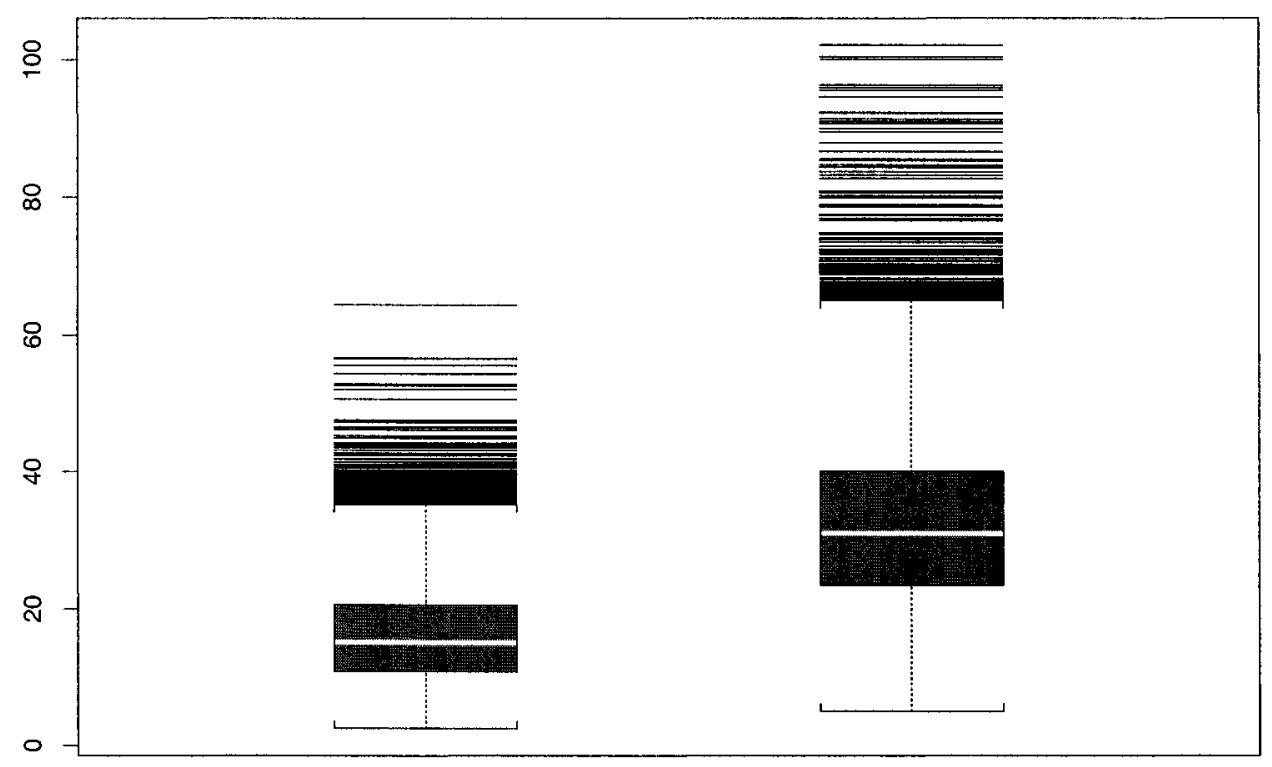

Figure 5.2: THIS BOXPLOT IS REFERRED TO THE RESUlT OF SIMULATION STUDY TO COMPARE THE PERFORMANCE OF THE RGAM FIT AND THE S-PLUS ROBUST FIT, WHEN THE SPAN $=0.5$ AND $\mathrm{K}=0.5$.

boxplots don't have identical median values. The variability of the RGAM fit is much less than the S-PLUS robust fit, and also the extreme values are closer to the median in the RGAM fit compare to S-PLUS robust fit. 


$$
\operatorname{span}=0.5
$$

\begin{tabular}{|c|c|c|c|c|}
\hline $\begin{array}{c}\text { Extreme } \\
\text { Center }\end{array}$ & $\begin{array}{c}\alpha=0.01 \\
\text { mean(sd) }\end{array}$ & $\begin{array}{c}\alpha=0.001 \\
\text { mean(sd) }\end{array}$ & $\begin{array}{c}\alpha=0.0005 \\
\text { mean(sd) }\end{array}$ & $\begin{array}{c}\alpha=0.0001 \\
\text { mean(sd) }\end{array}$ \\
\hline K=0.5 & \multicolumn{2}{|c|}{ M-RGAM fit=16.45(7.71) } & M-S-PLUS robust fit $=44.87(15.84)$ \\
\hline RPTO & $100(0.00)$ & $100(0.00)$ & $100(0.00)$ & $100(0.00)$ \\
SPTO & $100(0.00)$ & $100(0.00)$ & $100(0.00)$ & $100(0.00)$ \\
RPFO & $37.34(1.94)$ & $7.19(1.03)$ & $4.15(0.64)$ & $1.22(0.20)$ \\
SPFO & $33.63(1.96)$ & $5.56(0.83)$ & $3.08(0.47)$ & $0.79(0.13)$ \\
\hline K=1 & M-RGAM fit=16.11(7.31) & M-S-PLUS robust fit=55.32(16.81) \\
\hline RPTO & $100(0.00)$ & $100(0.00)$ & $100(0.00)$ & $100(0.00)$ \\
SPTO & $100(0.00)$ & $100(0.00)$ & $100(0.00)$ & $100(0.00)$ \\
RPFO & $36.45(1.19)$ & $6.83(0.98)$ & $3.91(0.60)$ & $1.13(0.18)$ \\
SPFO & $32.48(1.19)$ & $5.19(0.77)$ & $2.79(0.43)$ & $0.71(0.11)$ \\
\hline K=1.5 & M-RGAM fit=17.28(7.63) & M-S-PLUS robust fit=81.16(22.13) \\
\hline RPTO & $100(0.00)$ & $100(0.00)$ & $100(0.00)$ & $100(0.00)$ \\
SPTO & $100(0.00)$ & $100(0.00)$ & $100(0.00)$ & $100(0.00)$ \\
RPFO & $35.57(1.86)$ & $6.57(0.94)$ & $3.68(0.57)$ & $1.05(0.17)$ \\
SPFO & $30.81(1.84)$ & $4.52(0.67)$ & $2.42(0.37)$ & $0.56(0.09)$ \\
\hline
\end{tabular}

Table 3: Result of simulation to compare the average percentage of true outliers detection and false ALARM AS OBTAINED WITH RGAM AND S-PLUS ROBUST GAM FOR DIFFERENT VALUES OF THE TUNING CONSTANT K AND SPAN $=0.5$, WHEN EXTREME OUTLIERS ARE LOCATED IN CENTER OF THE RANGE OF THE COVARIATE. 


\begin{tabular}{|c|c|c|c|c|c|}
\hline Moderate & $\alpha=0.01$ & $\alpha=0.001$ & $\alpha=0.0005$ & $\alpha=0.0001$ \\
Upper end & mean(sd) & mean(sd) & mean(sd) & mean(sd) \\
\hline K=0.5 & M-RGAM fit=27.70(20.10) & M-S-PLUS robust fit=341.81(72.35) \\
\hline RPTO & $99.96(0.01)$ & $99.42(0.29)$ & $98.88(0.61)$ & $96.13(2.12)$ \\
SPTO & $9.19(2.33)$ & $0.664(0.16)$ & $0.288(0.07)$ & $0.048(0.009)$ \\
RPFO & $36.98(1.95)$ & $7.10(1.06)$ & $4.19(0.72)$ & $1.25(0.25)$ \\
SPFO & $85.24(6.3)$ & $30.76(21.09)$ & $18.67(15.33)$ & $4.71(4.48)$ \\
\hline K=1 & M-RGAM fit=31.12(21.59) & M-S-PLUS robust fit=316.93(50.08) \\
\hline RPTO & $99.96(0.008)$ & $99.12(0.47)$ & $98.47(0.8)$ & $94.54(2.9)$ \\
SPTO & $9.63(1.74)$ & $0.58(0.12)$ & $0.24(0.05)$ & $0.036(0.007)$ \\
RPFO & $36.42(1.9)$ & $6.86(1.05)$ & $4.02(0.67)$ & $1.24(0.29)$ \\
SPFO & $84.28(5.9)$ & $30.54(20.93)$ & $18.19(14.81)$ & $4.66(4.44)$ \\
\hline K=1.5 & \multicolumn{2}{|c|}{ M-RGAM fit=43.78(29.89) } & M-S-PLUS robust fit=281.58(40.59) \\
\hline RPTO & $99.7(0.13)$ & $97(1.67)$ & $94.7(2.83)$ & $86.14(7.19)$ \\
SPTO & $13.6(2.03)$ & $0.96(0.19)$ & $0.46(0.09)$ & $0.068(0.01)$ \\
RPFO & $35.98(1.88)$ & $6.99(1.3)$ & $4.1(0.86)$ & $1.31(0.4)$ \\
SPFO & $79.66(5.93)$ & $29.33(20.32)$ & $17.25(14.15)$ & $4.53(4.32)$ \\
\hline
\end{tabular}

Table 4: Result of simulation to compare the aVerage percentage of true outliers detection and False ALARM AS OBTAINED WITH RGAM AND S-PLUS ROBUST GAM FOR DIFFERENT VALUES OF THE TUNING CONSTANT K AND SPAN $=0.5$, WHEN MODERATE OUTLIERS ARE LOCATED IN END OF THE RANGE OF THE COVARIATE. 


$$
\operatorname{span}=0.5
$$

\begin{tabular}{|c|c|c|c|c|}
\hline $\begin{array}{c}\text { Extreme } \\
\text { Upper end }\end{array}$ & $\begin{array}{c}\alpha=0.01 \\
\operatorname{mean}(\mathrm{sd})\end{array}$ & $\begin{array}{l}\alpha=0.001 \\
\operatorname{mean}(\mathrm{sd})\end{array}$ & $\begin{array}{l}\alpha=0.0005 \\
\operatorname{mean}(\mathrm{sd})\end{array}$ & $\begin{array}{l}\alpha=0.0001 \\
\operatorname{mean}(\mathrm{sd})\end{array}$ \\
\hline $\mathrm{K}=0.5$ & \multicolumn{2}{|c|}{ M-RGAM fit $=27.71(20.12)$} & \multicolumn{2}{|c|}{ M-S-PLUS robust fit $=2184.28(236.8)$} \\
\hline RPTO & $100(0.00)$ & $100(0.00)$ & $100(0.00)$ & $100(0.00)$ \\
\hline SPTO & $35.1(2.1)$ & $6.96(1.16)$ & $3.32(0.61)$ & $0.47(0.09)$ \\
\hline RPFO & $36.98(1.95)$ & $7.02(1.01)$ & $4.08(0.62)$ & $1.16(0.19)$ \\
\hline SPFO & $60.9(3.9)$ & $27.71(17.35)$ & $18.26(14.15)$ & $5.08(4.79)$ \\
\hline $\mathrm{K}=1$ & \multicolumn{2}{|c|}{ M-RGAM fit=31.14(21.6) } & \multicolumn{2}{|c|}{ M-S-PLUS robust fit $=2040.78(172.43)$} \\
\hline RPTO & $100(0.00)$ & $100(0.00)$ & $100(0.00)$ & $100(0.00)$ \\
\hline SPTO & $40.42(1.54)$ & $11.57(1.4)$ & $5.96(0.91)$ & $0.86(0.16)$ \\
\hline RPFO & $36.41(1.9)$ & $6.72(0.97)$ & $3.88(0.59)$ & $1.1(0.18)$ \\
\hline SPFO & $56.87(3.26)$ & $23.28(13.46)$ & $16.04(12.07)$ & $4.87(4.58)$ \\
\hline $\mathrm{K}=1.5$ & \multicolumn{2}{|c|}{ M-RGAM fit=43.83(29.96) } & \multicolumn{2}{|c|}{ M-S-PLUS robust fit $=1872.05(143.56)$} \\
\hline RPTO & $100(0.00)$ & $100(0.00)$ & $100(0.00)$ & $100(0.00)$ \\
\hline SPTO & $46.56(1.25)$ & $19.09(1.45)$ & $11.84(1.35)$ & $2.3(0.42)$ \\
\hline $\mathrm{RPFO}$ & $35.89(1.85)$ & $6.52(0.93)$ & $3.7(0.56)$ & $1.02(0.16)$ \\
\hline SPFO & $53.13(2.9)$ & $18(8.6)$ & $12.65(8.51)$ & $4.32(4)$ \\
\hline
\end{tabular}

Table 5: Result of simulation to compare the aVerage Percentage of true outliers detection and false ALARM AS OBTAINED WITH RGAM AND S-PLUS ROBUST GAM FOR DIFFERENT VALUES OF THE TUNING CONSTANT K AND SPAN $=0.5$, WHEN EXTREME OUTLIERS ARE LOCATED IN END OF THE RANGE OF THE COVARIATE. 
$\operatorname{span}=0.25$

\begin{tabular}{|c|c|c|c|c|}
\hline $\begin{array}{c}\text { Moderate } \\
\text { Center }\end{array}$ & $\begin{array}{c}\alpha=0.01 \\
\operatorname{mean}(\mathrm{sd})\end{array}$ & $\begin{array}{l}\alpha=0.001 \\
\text { mean }(\mathrm{sd})\end{array}$ & $\begin{array}{l}\alpha=0.0005 \\
\text { mean }(\mathrm{sd})\end{array}$ & $\begin{array}{l}\alpha=0.0001 \\
\operatorname{mean}(\mathrm{sd})\end{array}$ \\
\hline$K=0.5$ & \multicolumn{2}{|c|}{ M-RGAM fit $=24.67(12.44)$} & \multicolumn{2}{|c|}{ M-S-PLUS robust fit $=44.17(17.95)$} \\
\hline RPTO & $100(0.00)$ & $100(0.00)$ & $100(0.00)$ & $99.95(0.02)$ \\
\hline SPTO & 100 & $99.98(0.0031)$ & $99.94(0.019)$ & $99.70(0.12)$ \\
\hline RPFO & $37.19(1.92)$ & $6.99(1.03)$ & $4.07(0.62)$ & $1.15(0.18)$ \\
\hline SPFO & $33.17(1.93)$ & $5.33(0.80)$ & $2.89(0.45)$ & $0.73(0.12)$ \\
\hline $\mathrm{K}=1$ & \multicolumn{2}{|c|}{ M-RGAM fit $=25.21(12.45)$} & \multicolumn{2}{|c|}{ M-S-PLUS robust fit $=61.39(22.52)$} \\
\hline RPTO & $100(0.00)$ & $100(0.00)$ & $100(0.00)$ & $99.96(0.01)$ \\
\hline SPTO & 100 & $99.94(0.01)$ & $99.83(0.05)$ & $97.04(0.93)$ \\
\hline RPFO & $35.71(1.88)$ & $6.47(0.95)$ & $3.55(0.55)$ & $0.98(0.16)$ \\
\hline SPFO & $31.70(1.90)$ & $4.96(0.74)$ & $2.66(0.42)$ & $0.68(0.11)$ \\
\hline$K=1.5$ & \multicolumn{2}{|c|}{ M-RGAM fit $=30.31(14.78)$} & \multicolumn{2}{|c|}{ M-S-PLUS robust fit $=78.40(24.83)$} \\
\hline RPTO & $100(0.00)$ & $100(0.00)$ & $99.99(0.0008)$ & $99.86(0.04)$ \\
\hline SPTO & 100 & $99.53(0.11)$ & $98.47(0.50)$ & $89.24(3.62)$ \\
\hline RPFO & $34.10(1.88)$ & $5.89(0.86)$ & $3.16(0.49)$ & $0.84(0.13)$ \\
\hline SPFO & $30.15(1.87)$ & $4.38(0.66)$ & $2.37(0.38)$ & $0.66(0.14)$ \\
\hline
\end{tabular}

Table 6: Result of simulation to COMPare the aVerage percentage of true outliers detection and False ALARM AS OBTAINED WITH RGAM AND S-PLUS ROBUST GAM FOR DIFFERENT VALUES OF THE TUNING CONSTANT K AND SPAN $=0.25$, WHEN MODERATE OUTLIERS ARE LOCATED IN CENTER. OF THE RANGE OF THE COVARIATE. 
$\operatorname{span}=0.25$

\begin{tabular}{|c|c|c|c|c|}
\hline $\begin{array}{c}\text { Extreme } \\
\text { Center }\end{array}$ & $\begin{array}{c}\alpha=0.01 \\
\text { mean(sd) }\end{array}$ & $\begin{array}{c}\alpha=0.001 \\
\text { mean(sd) }\end{array}$ & $\begin{array}{c}\alpha=0.0005 \\
\text { mean(sd) }\end{array}$ & $\begin{array}{c}\alpha=0.0001 \\
\text { mean(sd) }\end{array}$ \\
\hline K=0.5 & M-RGAM fit=24.68(12.45) & M-S-PLUS robust fit=108.42(148.44) \\
\hline RPTO & $100(0.00)$ & $100(0.00)$ & $100(0.00)$ & $100(0.00)$ \\
SPTO & $100(0.00)$ & $100(0.00)$ & $100(0.00)$ & $100(0.00)$ \\
RPFO & $37.18(1.92)$ & $7.00(1.03)$ & $4.07(0.62)$ & $1.15(0.18)$ \\
SPFO & $32.94(1.99)$ & $5.36(0.81)$ & $2.88(0.45)$ & $0.74(0.12)$ \\
\hline K=1 & M-RGAM fit=25.22(12.46) & M-S-PLUS robust fit=199.76(196.24) \\
\hline RPTO & $100(0.00)$ & $100(0.00)$ & $100(0.00)$ & $100(0.00)$ \\
SPTO & $100(0.00)$ & $100(0.00)$ & $100(0.00)$ & $100(0.00)$ \\
RPFO & $35.71(1.88)$ & $6.46(0.95)$ & $3.55(0.55)$ & $0.98(0.16)$ \\
SPFO & $31.05(1.95)$ & $4.86(0.73)$ & $2.55(0.40)$ & $0.64(0.10)$ \\
\hline K=1.5 & M-RGAM fit=30.33(14.79) & M-S-PLUS robust fit $=226.81(180.67)$ \\
\hline RPTO & $100(0.00)$ & $100(0.00)$ & $100(0.00)$ & $100(0.00)$ \\
SPTO & $100(0.00)$ & $100(0.00)$ & $100(0.00)$ & $100(0.00)$ \\
RPFO & $34.10(1.88)$ & $5.89(0.86)$ & $3.16(0.49)$ & $0.84(0.13)$ \\
SPFO & $29.16(1.89)$ & $4.10(0.62)$ & $2.18(0.34)$ & $0.54(0.08)$ \\
\hline
\end{tabular}

Table 7: Result of Simulation to compare the aVERaGE PERcentage of true outliers detection and False ALARM AS OBTAINED WITH RGAM AND S-PLUS ROBUST GAM FOR DIFFERENT VALUES OF THE TUNING CONSTANT K AND SPAN $=0.25$ WHEN EXTREME OUTLIERS ARE LOCATED IN CENTER OF THE RANGE OF THE COVARIATE. 


$$
\operatorname{span}=0.25
$$

\begin{tabular}{|c|c|c|c|c|}
\hline $\begin{array}{c}\text { Moderate } \\
\text { Upper end }\end{array}$ & $\begin{array}{c}\alpha=0.01 \\
\text { mean(sd) }\end{array}$ & $\begin{array}{c}\alpha=0.001 \\
\text { mean(sd) }\end{array}$ & $\begin{array}{c}\alpha=0.0005 \\
\text { mean(sd) }\end{array}$ & $\begin{array}{c}\alpha=0.0001 \\
\text { mean(sd) }\end{array}$ \\
\hline K=0.5 & \multicolumn{2}{|c|}{ M-RGAM fit=416.99(63.65) } & \multicolumn{2}{|c|}{ M-S-PLUS robust fit=453.87(59.04) } \\
\hline RPTO & $6.39(1.07)$ & $0.47(0.09)$ & $0.21(0.04)$ & $0.04(0.008)$ \\
SPTO & $1.54(0.30)$ & $0.06(0.01)$ & $0.02(0.005)$ & $0.008(0.001)$ \\
RPFO & $89.39(4.37)$ & $37.17(23.12)$ & $23.60(17.97)$ & $6.76(6.30)$ \\
SPFO & $93.12(5.06)$ & $30.97(21.37)$ & $17.82(14.64)$ & $4.58(4.37)$ \\
\hline K=1 & M-RGAM fit=409.85(55.45) & M-S-PLUS robust fit=411.02(50.69) \\
\hline RPTO & $6.77(1.10)$ & $0.53(0.10)$ & $0.20(0.04)$ & $0.04(0.008)$ \\
SPTO & $2.87(0.53)$ & $0.13(0.02)$ & $0.06(0.01)$ & $0.008(0.001)$ \\
RPFO & $88.59(4.53)$ & $35.34(22.63)$ & $21.33(16.73)$ & $6.24(5.85)$ \\
SPFO & $91.60(5.21)$ & $30.80(21.28)$ & $17.98(14.74)$ & $4.92(4.67)$ \\
\hline K=1.5 & \multicolumn{2}{|c|}{ M-RGAM fit=393.06(51.11) } & M-S-PLUS robust fit=374.74(45.50) \\
\hline RPTO & \multicolumn{2}{|c|}{$7.54(1.17)$} & $0.61(0.12)$ & \multicolumn{2}{|c|}{$0.24(0.05)$} & $0.04(0.008)$ \\
SPTO & $4.31(0.74)$ & $0.23(0.04)$ & $0.09(0.01)$ & $0.01(0.003)$ \\
RPFO & $86.78(5.14)$ & $32.84(21.82)$ & $19.30(15.52)$ & $5.52(5.21)$ \\
SPFO & $89.73(5.47)$ & $29.63(20.77)$ & $17.28(14.28)$ & $4.46(4.26)$ \\
\hline
\end{tabular}

Table 8: Result of Simulation to COMPare the aVerage percentage of tRUE outliers deteCtion and False ALARM AS OBTAINED WITH RGAM AND S-PLUS ROBUST GAM FOR DIFFERENT VALUES OF THE TUNING CONSTANT $\mathrm{K}$ AND SPAN $=0.25$, WHEN MODERATE OUTLIERS ARE LOCATED IN END OF THE RANGE OF THE COVARIATE. 
$\operatorname{span}=0.25$

\begin{tabular}{|c|c|c|c|c|}
\hline $\begin{array}{c}\text { Extreme } \\
\text { Upper end }\end{array}$ & $\begin{array}{c}\alpha=0.01 \\
\text { mean(sd) }\end{array}$ & $\begin{array}{c}\alpha=0.001 \\
\text { mean(sd) }\end{array}$ & $\begin{array}{c}\alpha=0.0005 \\
\text { mean(sd) }\end{array}$ & $\begin{array}{c}\alpha=0.0001 \\
\text { mean(sd) }\end{array}$ \\
\hline K=0.5 & \multicolumn{2}{|l|}{ M-RGAM fit=2092.19(223.47) } & \multicolumn{2}{|c|}{ M-S-PLUS robust fit=2475.89(182.98) } \\
\hline RPTO & $44.35(0.77)$ & $29.96(1.05)$ & $25.42(0.94)$ & $16.82(0.95)$ \\
SPTO & $26.22(1.09)$ & $5.52(0.83)$ & $2.43(0.43)$ & $0.26(0.05)$ \\
RPFO & $57.11(2.78)$ & $17.64(5.55)$ & $11.83(4.65)$ & $4.25(2.68)$ \\
SPFO & $64.64(4.17)$ & $26.74(17.24)$ & $16.88(13.40)$ & $4.58(4.36)$ \\
\hline K=1 & M-RGAM fit=2163.19(150.50) & M-S-PLUS robust fit=2359.009(153.65) \\
\hline RPTO & $42.76(0.51)$ & $28.91(1.01)$ & $24.58(0.79)$ & $16.62(0.84)$ \\
SPTO & $31.09(1.08)$ & $10.93(1.11)$ & $5.97(0.0086)$ & $0.91(0.17)$ \\
RPFO & $56.79(2.71)$ & $17.02(5.53)$ & $10.68(4.25)$ & $3.84(2.42)$ \\
SPFO & $61.58(3.67)$ & $22.93(13.38)$ & $15.51(11.73)$ & $4.75(4.46)$ \\
\hline K=1.5 & \multicolumn{2}{|l|}{ M-RGAM fit=2194.21(147.12) } & M-S-PLUS robust fit=2285.86(132.95) \\
\hline RPTO & $42.44(0.45)$ & $29.05(1.00)$ & $24.69(0.77)$ & $17.16(0.75)$ \\
SPTO & $34.37(0.89)$ & $15.28(0.98)$ & $9.76(1.06)$ & $1.87(0.34)$ \\
RPFO & $55.57(2.77)$ & $15.69(5.17)$ & $9.68(3.88)$ & $3.32(2.02)$ \\
SPFO & $58.62(3.53)$ & $19.01(9.92)$ & $13.24(9.41)$ & $4.24(3.95)$ \\
\hline
\end{tabular}

Table 9: Result of simulation to compare the average percentage of true outliers detection and false ALARM AS OBTAINED WITH RGAM AND S-PLUS ROBUST GAM FOR DIFFERENT VALUES OF THE TUNING CONSTANT K AND SPAN=0.25, WHEN EXTREME OUTLIERS ARE LOCATED IN END OF THE RANGE OF THE COVARIATE.

Note that the role that the position of outliers plays in the fit of each of the methods depends on its placement relative to other data. For example when the outliers are in center of the range of the covariate, as seen in Tables $2,3,6$ and 7 
M-RGAM fit remained in the range of 16.4 to 17.2 , and 24.6 to 30.3 by using the $\operatorname{span}=0.5$ and $\operatorname{span}=0.25$ respectively as the $K_{\mathrm{s}}$ changed from 0.5 to 1.5 , whereas the M-S-PLUS robust fit ranged from 32.9 to 81.1 and 44.1 to 226.8 by using the equivalent span of 0.5 and 0.25 respectively for the above Ks. Also, M-RGAM fit remains almost unchanged when the distance of the outliers increase or decrease from the curve line. But, the distance of the outliers from the curve noticeably changes the M-S-PLUS robust fit. However, when the outliers located in end of the range of the covariate, the same Ks and spans produce a range that is much larger for the S-PLUS robust fit than RGAM fit. Note, this evaluation took the form of 24 different runs for each model according to the various span, Ks and the placement of the outliers. The results of these simulations present RGAM fit give a better fit than S-PLUS robust fit except when the span is $0.25, \mathrm{k}=1.5$, the moderate outliers are located in end of the range of the covariate.

For the detection of outliers, when the outliers are located in center of the range of the covariate, both models can recognize the outlier with reasonable accuracy although RGAM fit is superior in its ability to detect but the percentage of mean of false alarms is higher in RGAM fit compare to S-PLUS robust fit. When the outliers located in end of the range of the covariate, the detection power of the S-PLUS robust fit falls considerably short of that of RGAM fit and the percentage of mean of false alarms is much lower in RGAM fit.

Note that when the isolated outliers are presented in the data, the accuracy although RGAM fit in detection of outliers is higher compare to a little cluster of the outliers; specially when a cluster of outliers are located in the end of the range of the covariate.

Hence, for all distances and placements of outliers, the RGAM fit outperforms the 
S-PLUS robust fit.

The first following graph illustrates both fits when the outliers are located in center of the range of the covariate, it implies the outliers change noticeably the S-PLUS robust fit. The curve fit which has been identified by dot line is the true mean, now we can see the closeness of RGAM fit to the true mean compare to the S-PLUS robust fit. The second one shows both fits when the outliers are located in upper end of the range of the covariate, the outliers have influence on both but the effect of the outliers on S-PLUS robust fit is much higher than the RGAM fit in this case. As the same as Figure 5.3 dot line has been identified by the true mean and we can see the closeness of RGAM fit to the true mean compare to the S-PLUS robust fit in this case. 


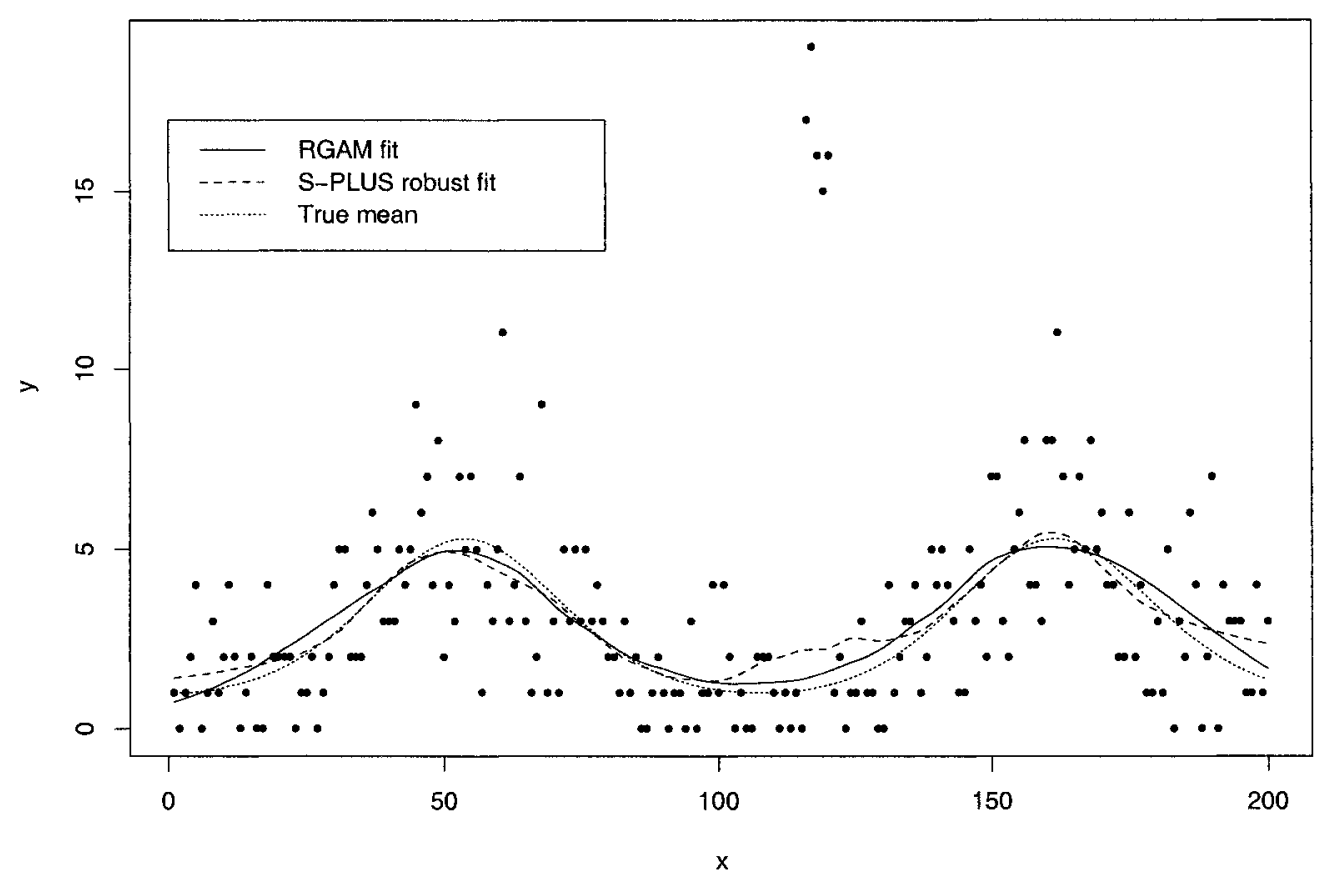

Figure 5.3: THIS GRAPH SHOWS THE CLOSENESS OF OUR FIT TO THE TRUE MEAN COMPARE TO THE GAM FIT WHEN THE OUTLIERS FALL IN THE MDDLE OF THE CURVE FIT. 


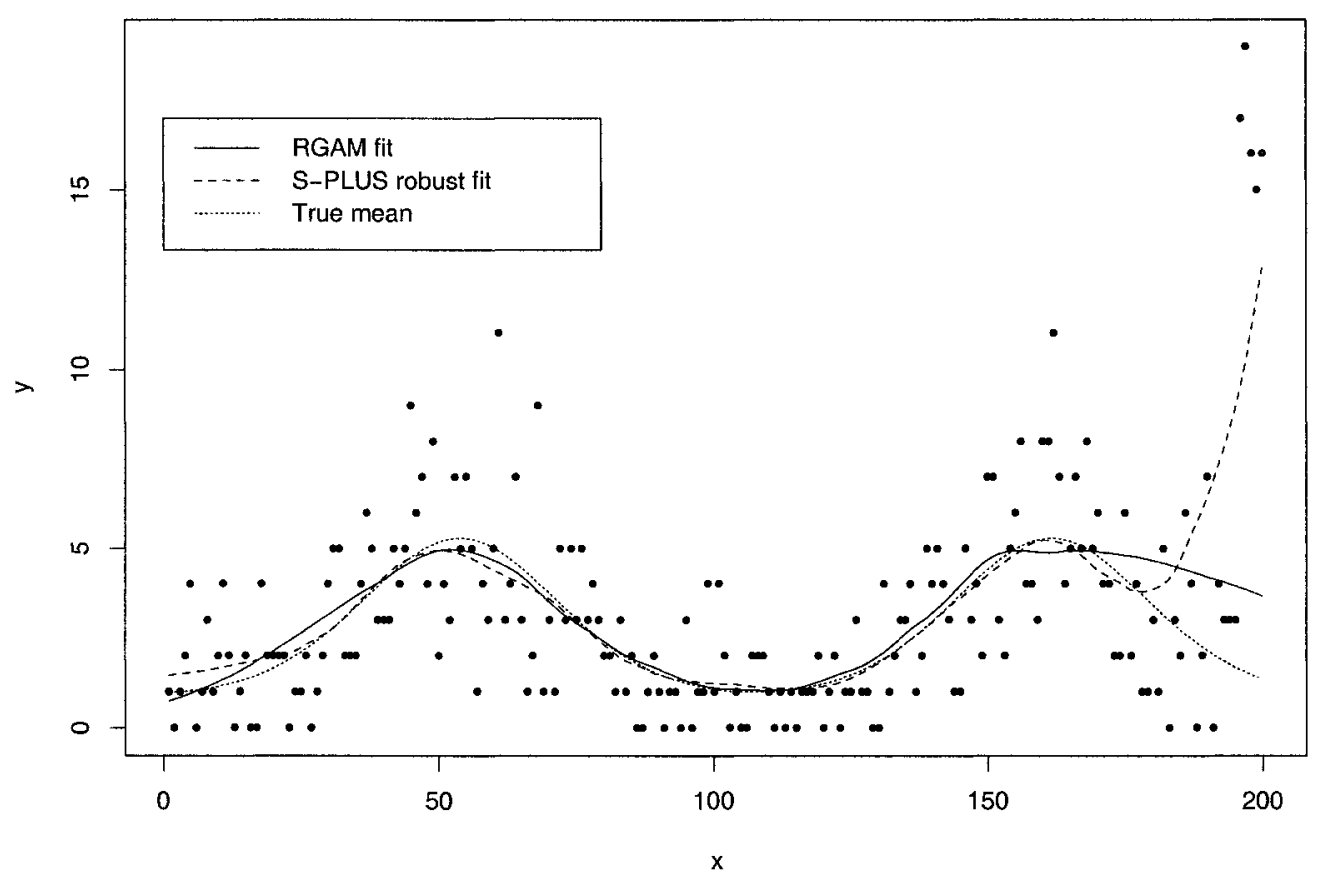

Figure 5.4: THIS GRAPH SHOWS THE CLOSENESS OF OUR. FIT TO THE TRUE MEAN COMPare tO THE GAM FIT WHEN THE OUTLIERS FALL TOWARD THE END OF THE FIT. 


\section{Chapter 6}

\section{Example}

The data used for this thesis originated from the database of the Public Health Information System of the BC Centre for Disease Control (BCCDC). This data contains weekly new cases of hepatitis C from 8 separate health regions in British Columbia for 262 weeks. The health regions included were: CRD: Capital health region, VRB: Vancouver Richmond health board, SFR: South Fraser region, PHC: Simon fraser health, FRV: Fraser valley health, THP: Thompson Okanagan, CVI: Central Vancouver island and OKS: Okanagan Similkameen. Table 10 contains a subset of these data.

\begin{tabular}{|c|c|c|c|c|c|c|c|c|}
\hline Date & CRD & VRB & SFR & PHC & FRV & THP & CVI & OKS \\
\hline $02 / 16 / 1998$ & 10 & 35 & 20 & 15 & 8 & 11 & 7 & 5 \\
$02 / 23 / 1998$ & 10 & 29 & 14 & 9 & 9 & 7 & 8 & 4 \\
$03 / 02 / 1998$ & 22 & 37 & 15 & 16 & 17 & 6 & 9 & 3 \\
$03 / 09 / 1998$ & 9 & 36 & 12 & 11 & 5 & 4 & 7 & 6 \\
\hline
\end{tabular}

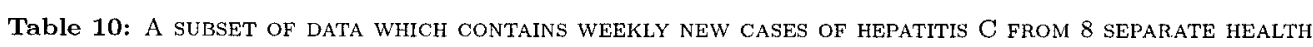
REGIONS IN BRITISH COLUMBIA FOR 262 WEEKS. 
One of the questions of interest is to detect outbreaks of hepatitis C. Because of the characteristics of these data, some time dependence may be expected. To check for this, we used the ACF plot on the residuals (the difference between what is actually observed and the predicted mean) for each region separately and the results show there is no time dependency in this experiment therefore we can assume that the observations were independent which is a required assumption in the GAM model. In our analysis each region is treated separately and contained 262 observations where $\left(Y_{i} \mid X_{i}\right) \sim P\left(\mu_{i}\right)$ where $Y_{i}$ is the number of weekly new cases of hepatitis $\mathrm{C}$ in the $i^{\text {th }}$ week and $X_{i}$ is the $i^{\text {th }}$ week in 262 weeks. We used an algorithm of outlier detection on this database to identify potential hepatitis $\mathrm{C}$ outbreaks in these regions and made a comparison between the RGAM fit and the S-PLUS robust fit. In this experiment, loess smoothers with span $=0.5$ in RGAM fit seem to provide a good fit based on the plots. Hence to get the similar fits in S-PLUS robust fit we consider span $=0.2$ in this case (see Section (5.1)). In this algorithm we obtain predicted means from both fits in each region and by using the responses we can identify the observations with tail probability less than $0.01,0.001,0.0005$ and 0.0001 which we label them as outliers . In the following plots $M$ is used to show the days which we detect as outliers using the S-PLUS robust fit and $\mathrm{O}$ is used to show the days which we detect the outliers using the RGAM fit in each region for $\alpha=0.01,0.001,0.0005$ and 0.0001 . Note each identified day by $\mathrm{M}$ or $\mathrm{O}$ are correspond to one of the 262 weeks. 

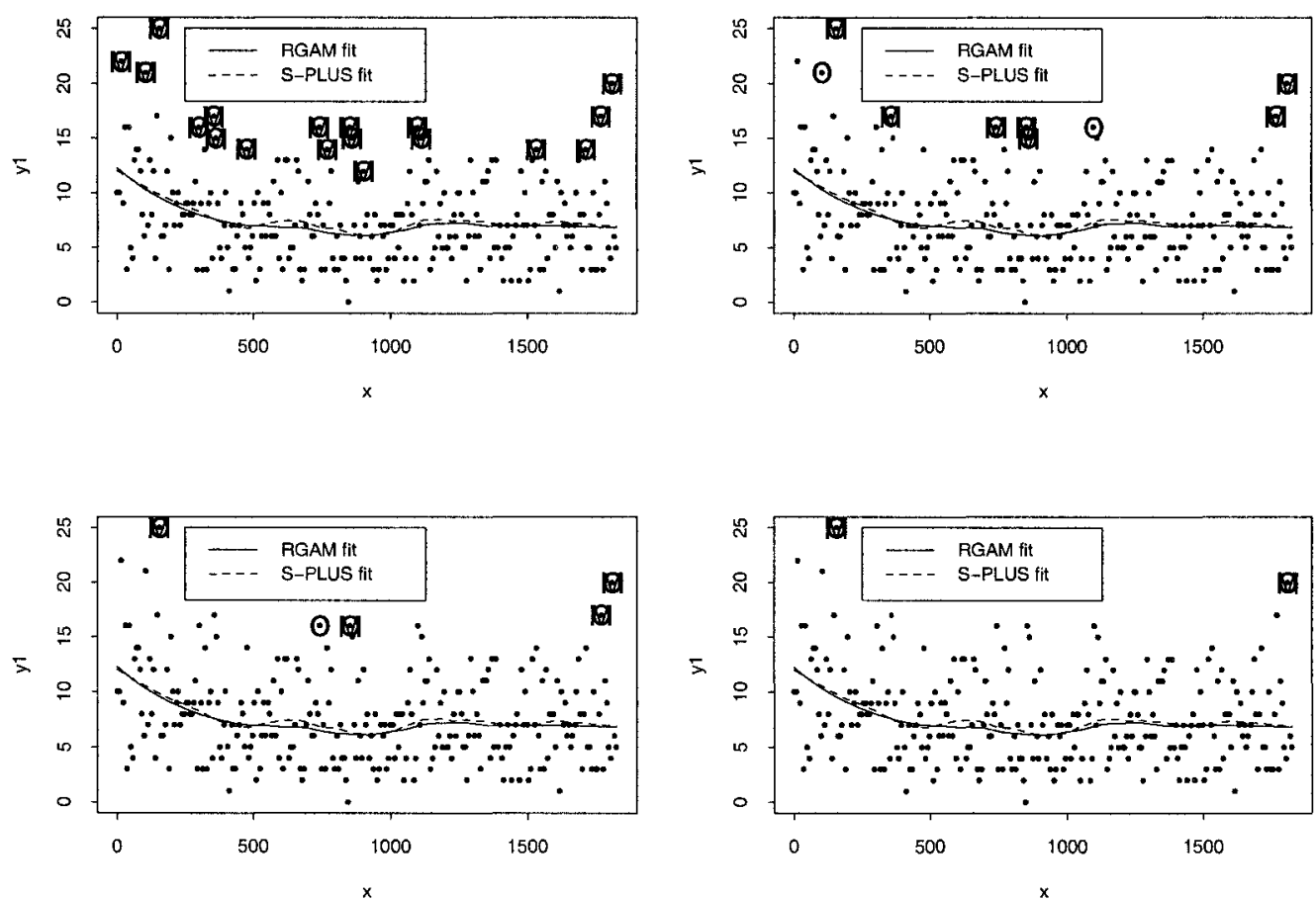

Figure 6.1: THE RESULT OF APPLYING RGAM FIT WITH SPAN $=0.5$ AND S-PLUS ROBUST GAM FIT WITH SPAN $=0.2$ WHEN $K=1$ AND $\alpha=0.01, \alpha=0.001, \alpha=0.0005, \alpha=0.0001$ RESPECTIVELY ON CRD REGION.

Both fits are applied and the results of outlier detection in these fits are the same for $\alpha=0.01$ and $\alpha=0.0001$ which is the largest and smallest tail probability that we considered in this experiment. When we consider $\alpha=0.001$ and $\alpha=0.0005$ both fits have missed a few points which is reasonable and it's caused of using the smaller $\alpha$. But the S-PLUS robust fit has missed a couple of points more than RGAM fit which is a result of the impact of outliers on this fit.

In this region seems outliying points have some effects on the S-PLUS robust fit and push the curve fit up, therefore it has missed a few point for all the values of $\alpha$ that we considered. 

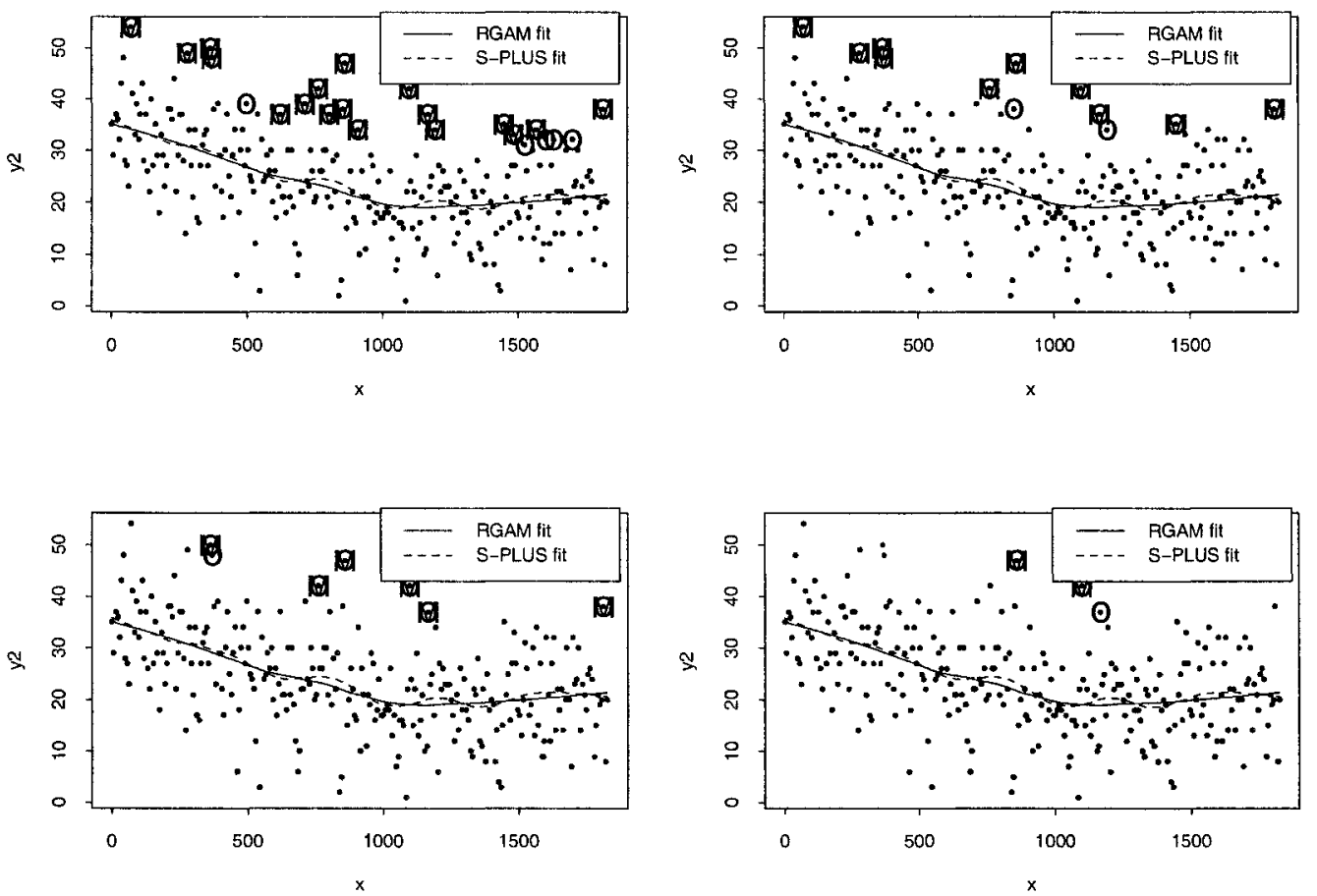

Figure 6.2: The Result of APPLYING RGAM Fit WITH SPAN $=0.5$ AND S-PLUS ROBUST GAM FIT WITH SPAN $=0.2$ WHEN $\mathrm{K}=1$ AND $\alpha=0.01, \alpha=0.001, \alpha=0.0005, \alpha=0.0001$ RESPECTIVELY ON VRB REGION. 

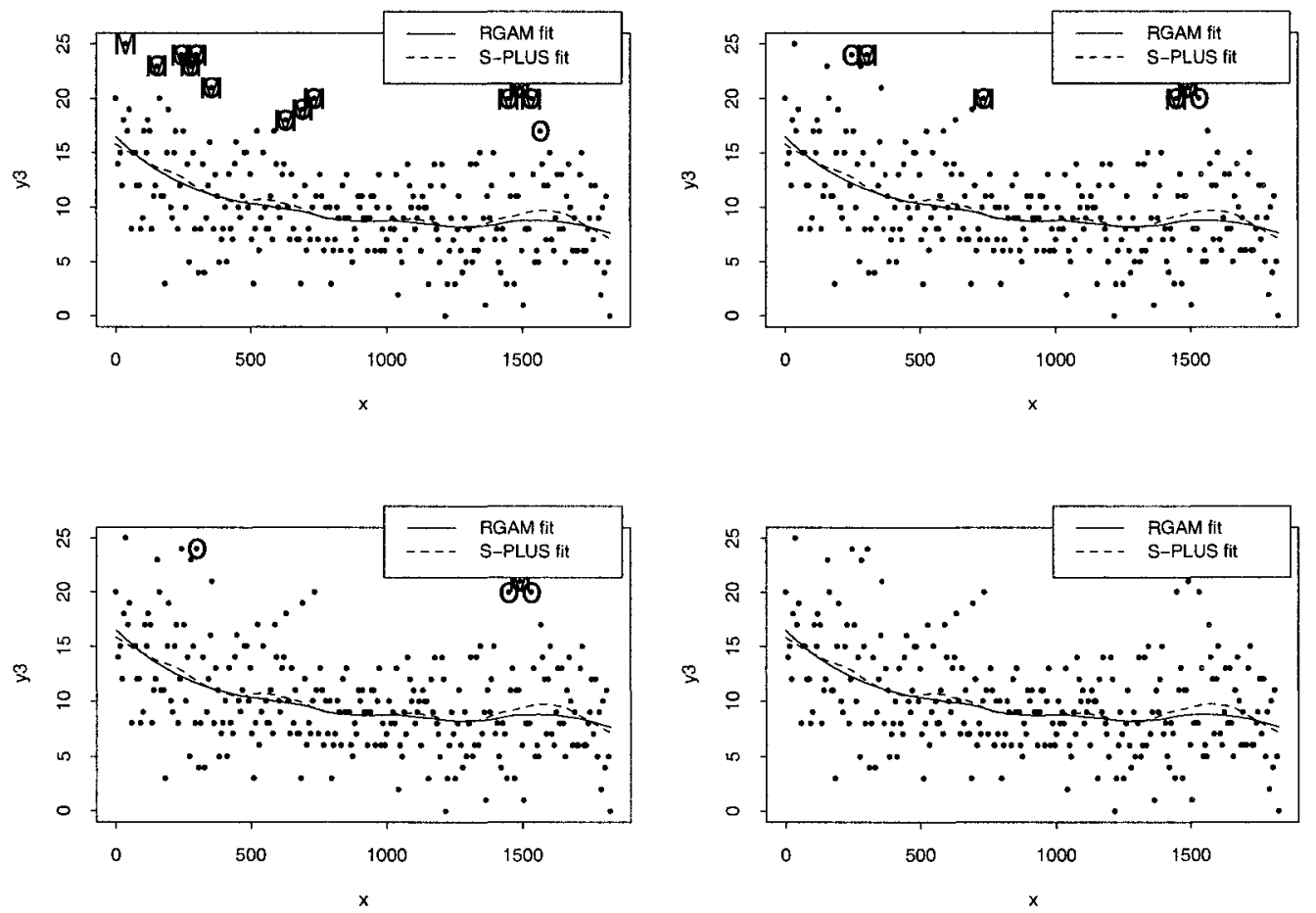

Figure 6.3: The RESULT OF APPLYING RGAM FIT WITH SPAN $=0.5$ AND S-PLUS ROBUST GAM FIT WITH SPAN=0.2 WHEN $K=1$ AND $\alpha=0.01, \alpha=0.001, \alpha=0.0005, \alpha=0.0001$ RESPECTIVELY ON SFR REGION.

In this case when $\alpha=0.01$ the RGAM fit has not detected the outliers in which is happened in the beginning. The S-PLUS robust GAM fit has missed some points because of the noticeable shift in the curve and when we used $\alpha=0.0001$ both fits predict there is no outliers in this region, therefore we may assume that there is no extreme outliers in this region. 

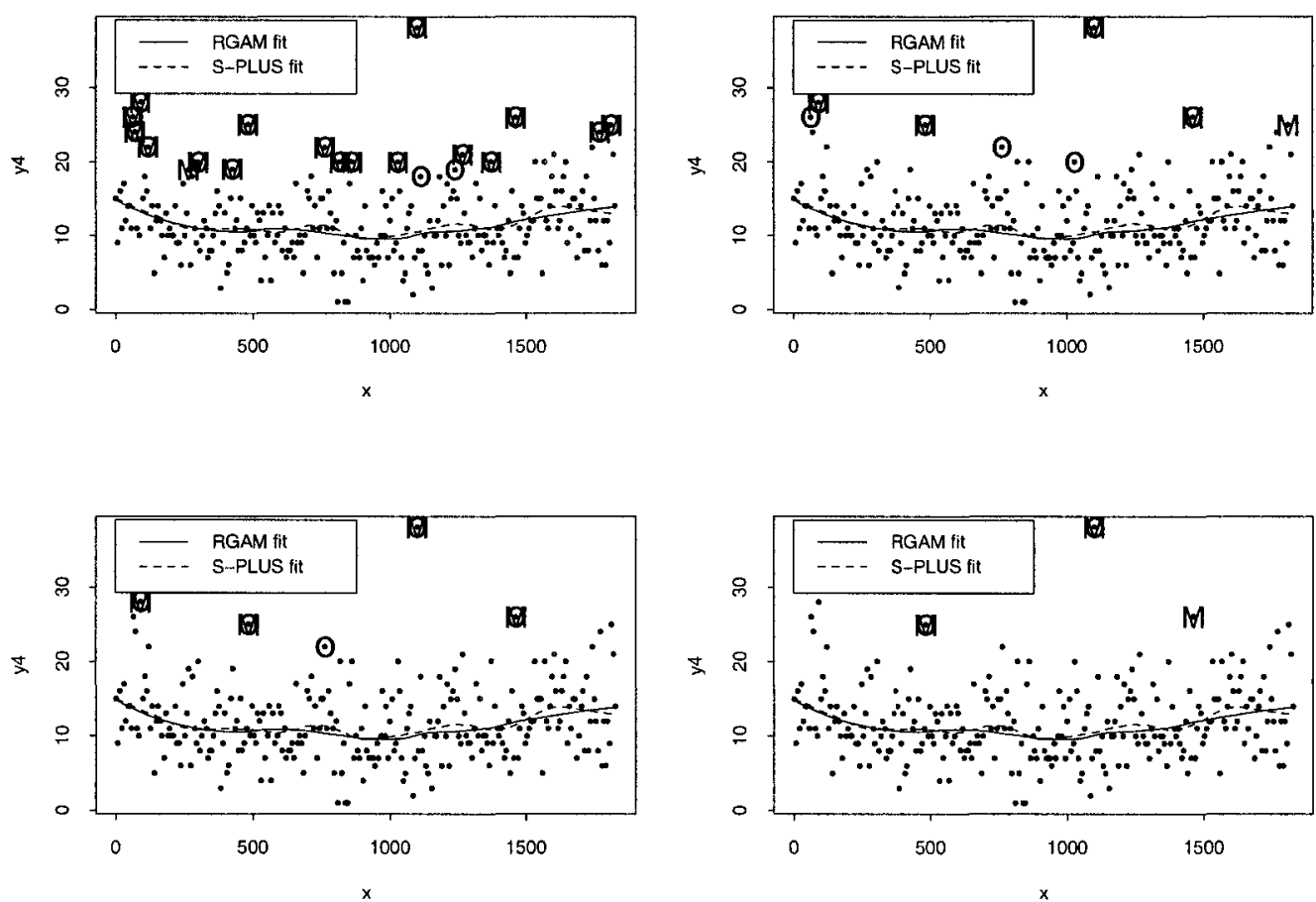

Figure 6.4: The RESUlt of APPLYING RGAM Fit With SPAN $=0.5$ AND S-PLUS RobUst GAM FIT With SPAN $=0.2$ WHEN $\mathrm{K}=1$ AND $\alpha=0.01, \alpha=0.001, \alpha=0.0005, \alpha=0.0001$ RESPECTIVELY ON PHC REGION

The number of new cases are high in 260 and 261-th weeks and it dropped in the last week, in the end of the curve fit the RGAM fit affected by outliers more than the S-PLUS robust GAM fit but in the middle of the curve fit outliers have more effect on the S-PLUS robust GAM fit compare to the RGAM fit which is a cause of missing some points by two fits. 

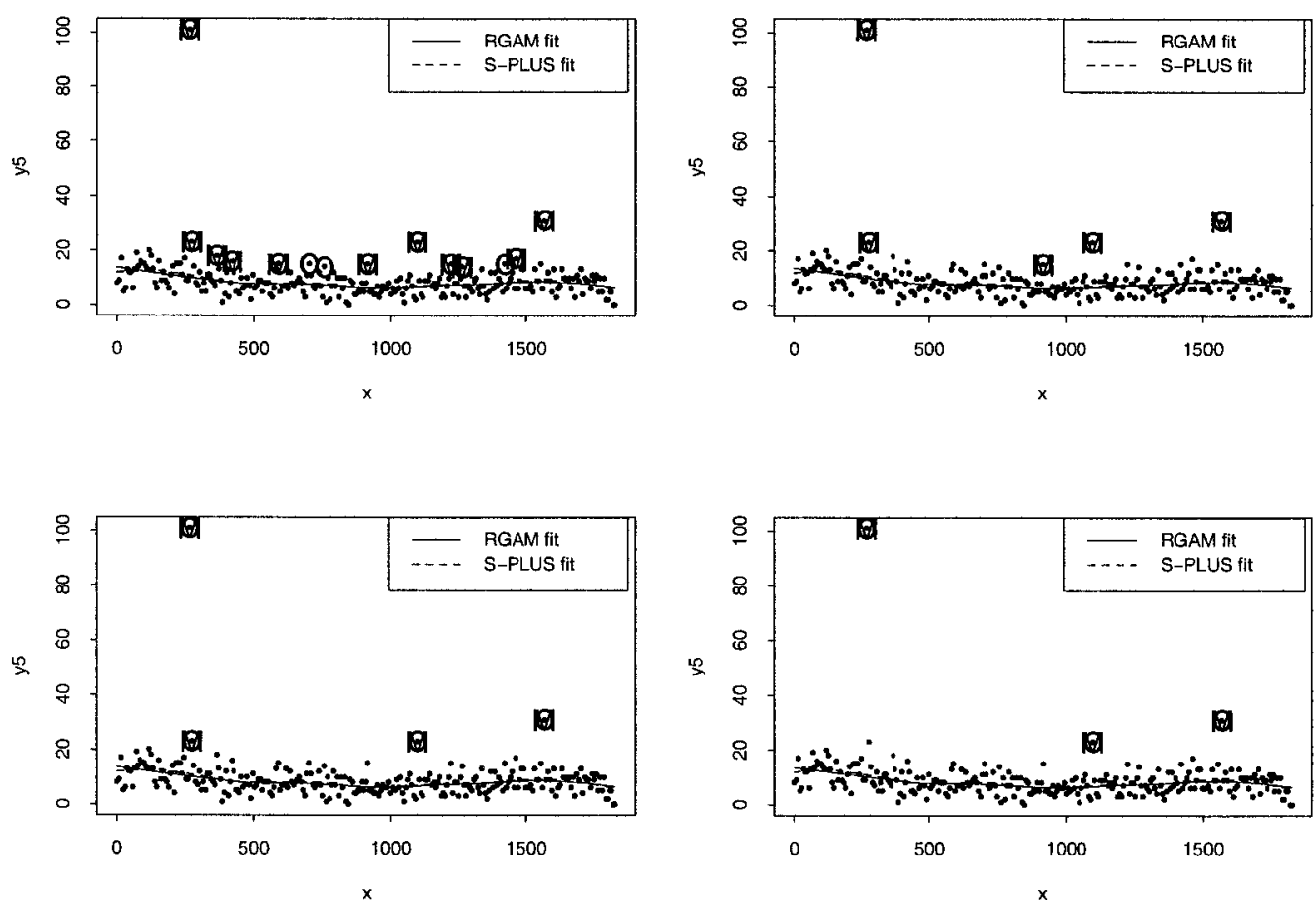

Figure 6.5: The RESULT OF APPLYING RGAM FIT WITH SPAN=0.5 AND S-PLUS ROBUST GAM FIT WITH SPAN=0.2 WHEN $\mathrm{K}=1$ AND $\alpha=0.01, \alpha=0.001, \alpha=0.0005, \alpha=0.0001$ RESPECTIVELY ON FRV REGION.

The number of new cases in the 39-th week (266-th days) is not reasonable compare to the next and previous week, therefore probably a false detection is happen in this case which could be a cause of typing error, etc. 

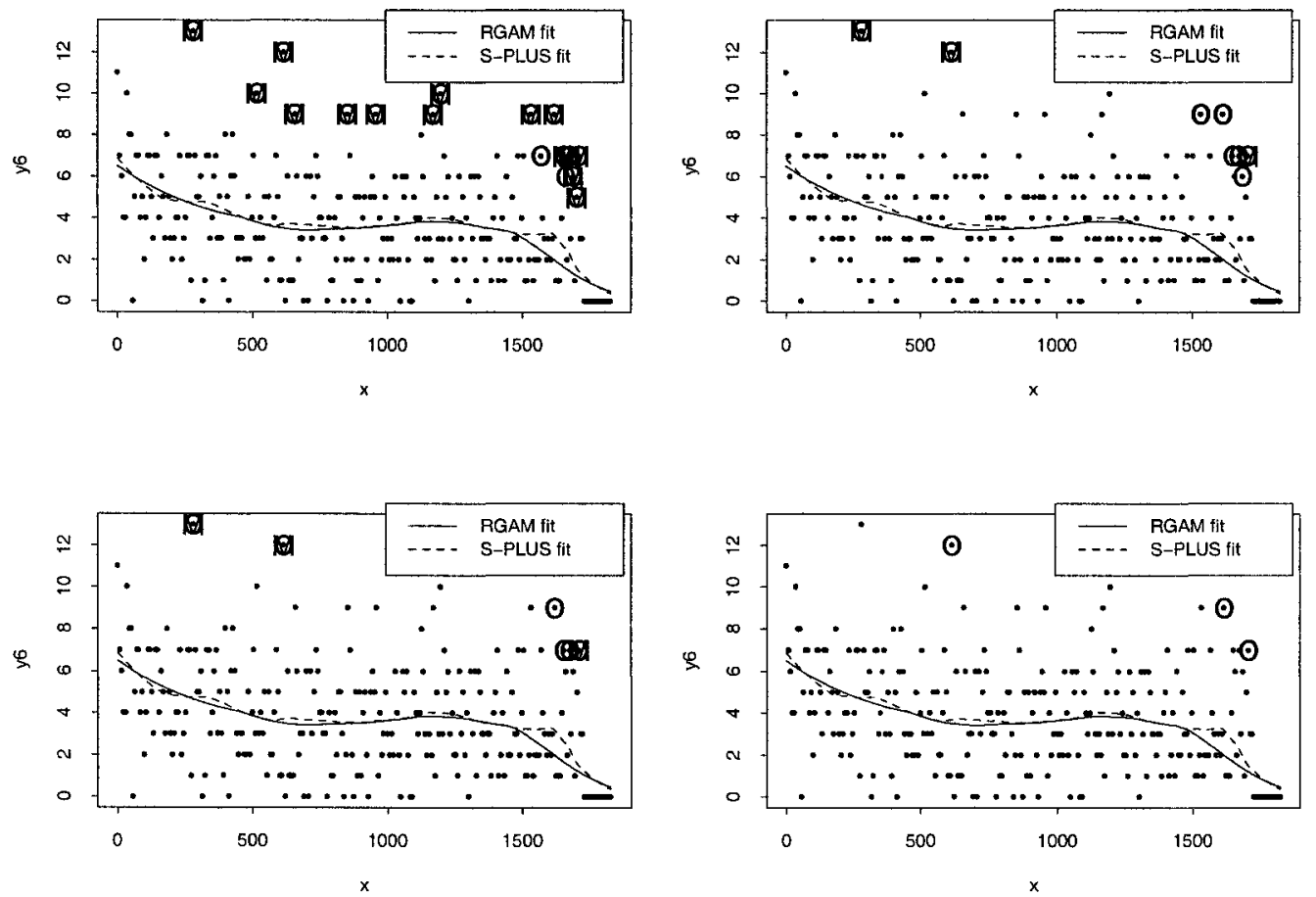

Figure 6.6.1: THE RESULT OF APPLYING RGAM FIT WITH SPAN $=0.5$ AND S-PLUS ROBUST GAM FIT WITH SPAN $=0.2$ WHEN $\mathrm{K}=1$ AND $\alpha=0.01, \alpha=0.001, \alpha=0.0005, \alpha=0.0001$ RESPECTIVELY ON THP REGION.

In this case the outliers at the end of the curve fit have an impact on the S-PLUS robust GAM fit and push it up noticeably, thus the RGAM fit detect the outliers much better than the S-PLUS robust GAM fit. Also there are just too many zeroes at the end of the data this may be is a recording mistake, we remove them and consider just the first 247 weeks. It shows the shape of graph is changed obviously therefore they consider less points as the outliers, the result shows in the figure 6.6.2. 

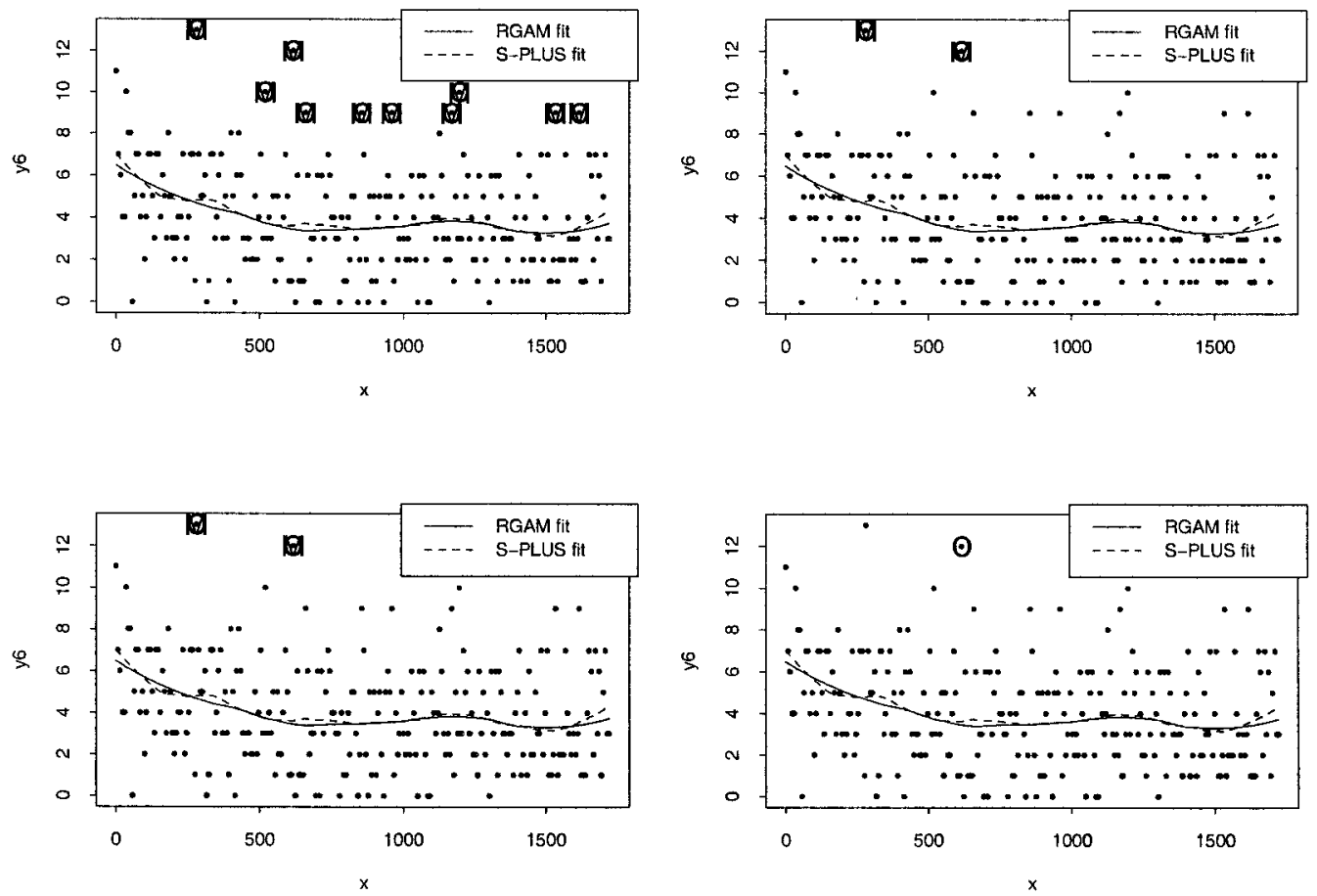

Figure 6.6.2: THE RESULT OF APPLYING RGAM FIT WITH SPAN $=0.5$ AND S-PLUS ROBUST GAM FIT WITH SPAN $=0.2$ WHEN $K=1$ AND $\alpha=0.01, \alpha=0.001, \alpha=0.0005, \alpha=0.0001$ RESPECTIVELY ON THP REGION FOR FIRST 247 WEEKS. 

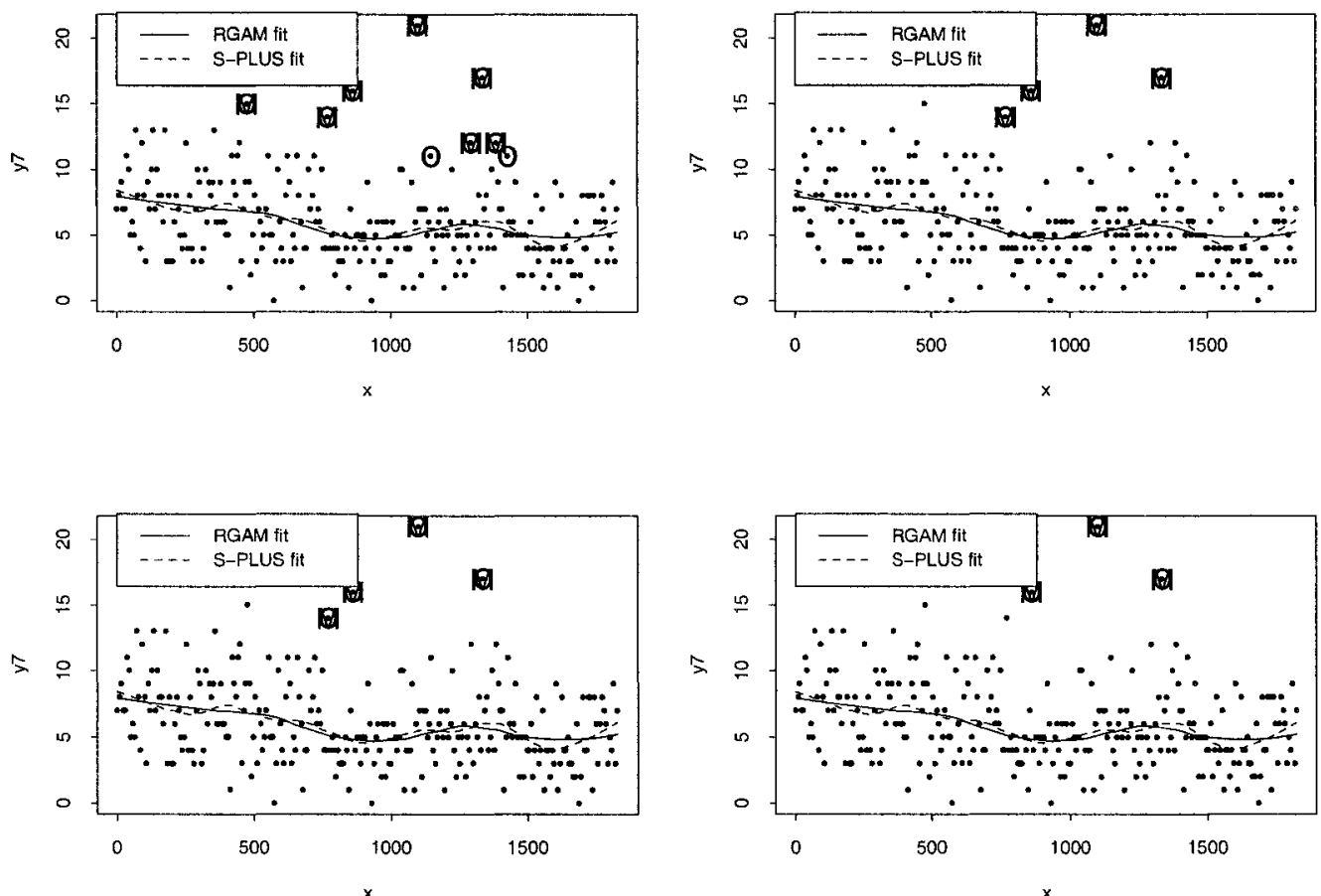

Figure 6.7: THE RESULT OF APPLYING RGAM FIT WITH SPAN $=0.5$ AND S-PLUS R.OBUST GAM FIT WITH SPAN=0.2 WHEN $K=1$ AND $\alpha=0.01, \alpha=0.001, \alpha=0.0005, \alpha=0.0001$ RESPECTIVELY ON CVI REGION.

The number of new cases in 260-th week jumps up compare to a few previous weeks and the next couple weeks, therefore it affects on the S-PLUS robust GAM fit and push it up. Fortunately, since there was no outliers at the end of data it does not any impact on the results. 

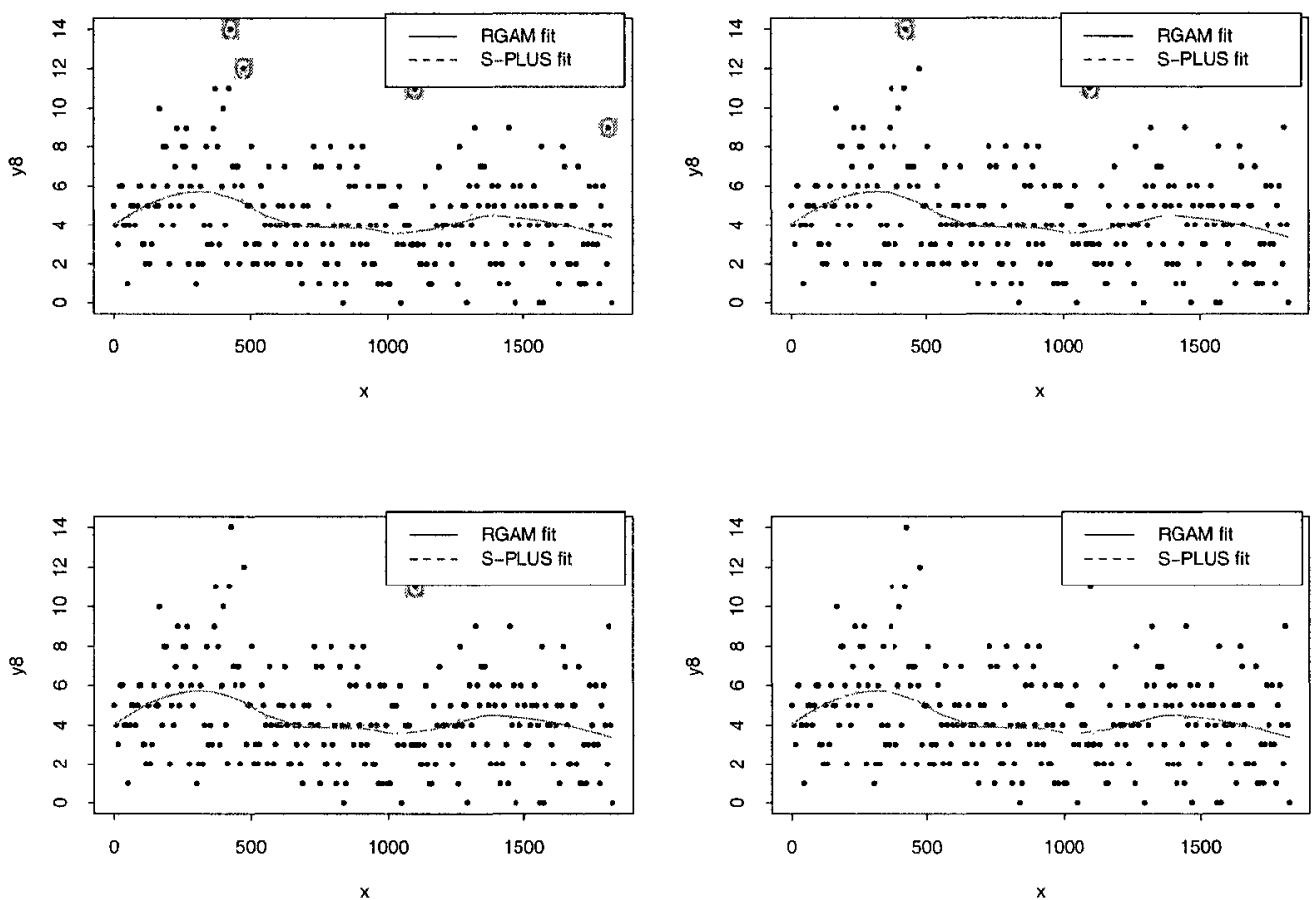

Figure 6.8: The ResUlt of APPLYING RGAM FIT With SPAN $=0.5$ AND S-PLUS ROBUST GAM FIT WITH SPAN $=0.2$ WHEN $\mathrm{K}=1$ AND $\alpha=0.01, \alpha=0.001, \alpha=0.0005, \alpha=0.0001$ RESPECTIVELY ON OKS REGION

In this case both fits work the same, also when we used $\alpha=0.0001$ both fits predict there is no outliers in this region.

In general based on the above graphs, if there are isolated cases of the outliers the behavior of both fits are similar except in the case that there is an extreme outlier which has an impact on S-PLUS robust GAM fit. Also if there are isolated cases of a little cluster of the outliers the behavior of both fits seem different. 


\section{Chapter 7}

\section{Appendix}

This chapter contains an implementation in S-PLUS of the algorithms presented in this thesis. It includes 2 separate programs. The first one is based on the Back Fitting Algorithm [9] which is a general algorithm that can fit an additive model using any regression-type fitting mechanisms. An additive model with response variable $Y$ and explanatory variables $X_{1}, \cdots, X_{p}$ has the form

$$
Y=\alpha+\sum_{j=1}^{p} f_{j}\left(X_{j}\right)+\varepsilon,
$$

where the errors $\varepsilon$ are independent of the $X_{j}$ s and $f_{j}$ is an arbitrary smooth function of the predictor $X_{j}$. We use LOESS, to estimate the $f_{j}$ s and we will identify them with $S$ in the following program.

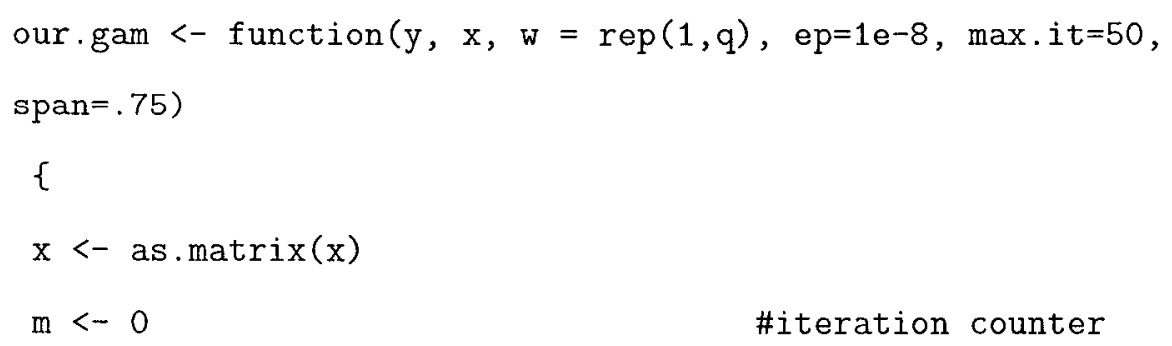




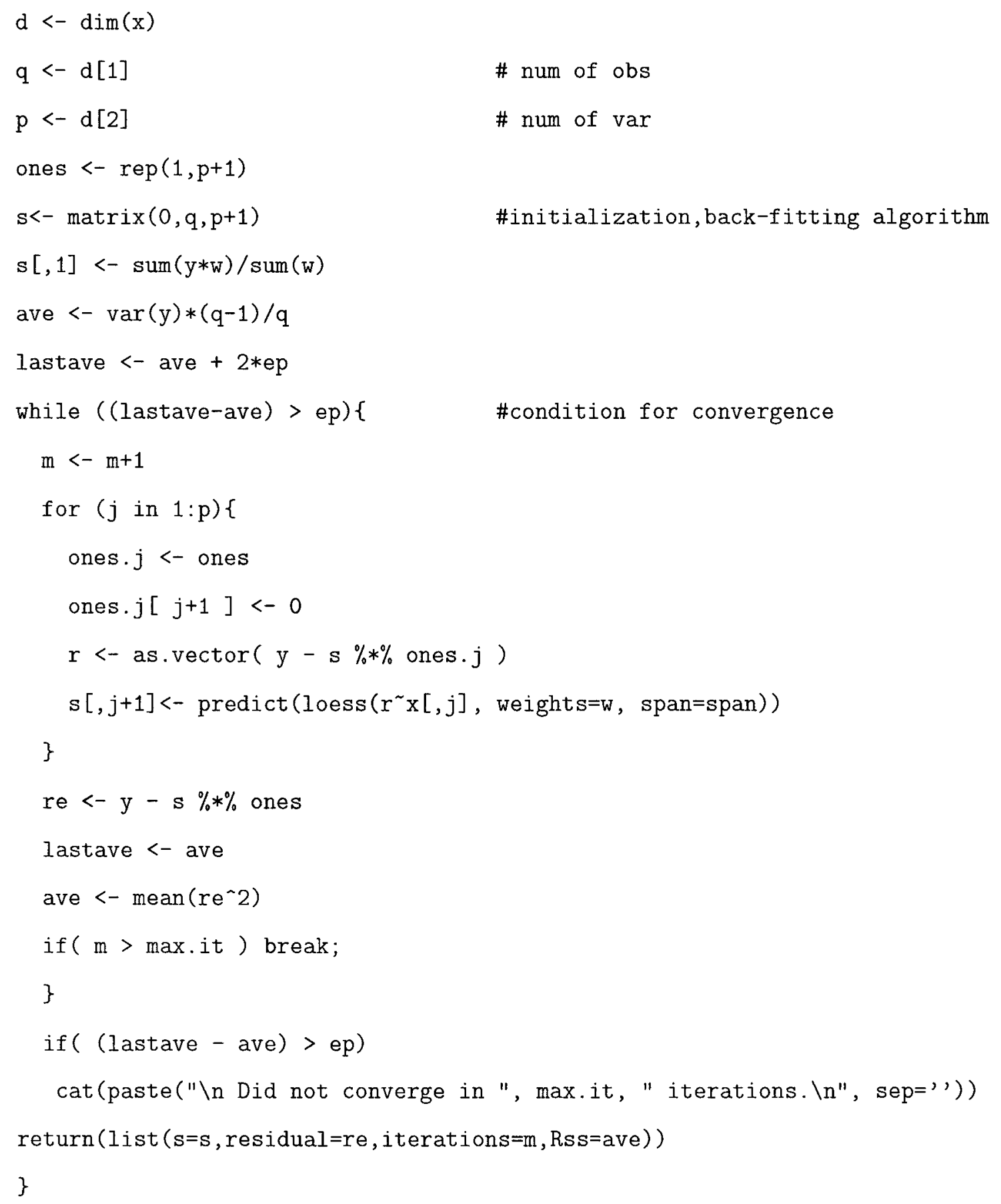


The second program is based on an algorithm to obtain a robust fit for GAM (see Section (4.3)), when the responses following Poisson distribution. In this case psi denote the Huber function (see equation 3.6) where $r=(y-\mu) / V^{1 / 2}(\mu)$ is the Pearson residual and $K$ denote the tuning constant of the $\psi$ function. We defined function norm to compute the convergence criterion based on the algorithm.

Since we use the General Local Scoring Algorithm (see Section (2.6)) with weights and adjusted responses derived from robust quasi score functions (see Section (4.3)), we need to compute $E\left(\psi_{k}\left(r_{i}\right)\right), E\left(\psi_{k}\left(r_{i}\right)\right), E\left(\psi_{k}^{\prime}\left(r_{i}\right) r_{i}\right)$ and $E\left(\frac{\partial}{\partial \eta_{i}} E\left(\psi_{k}\left(r_{i}\right)\right)\right)$.

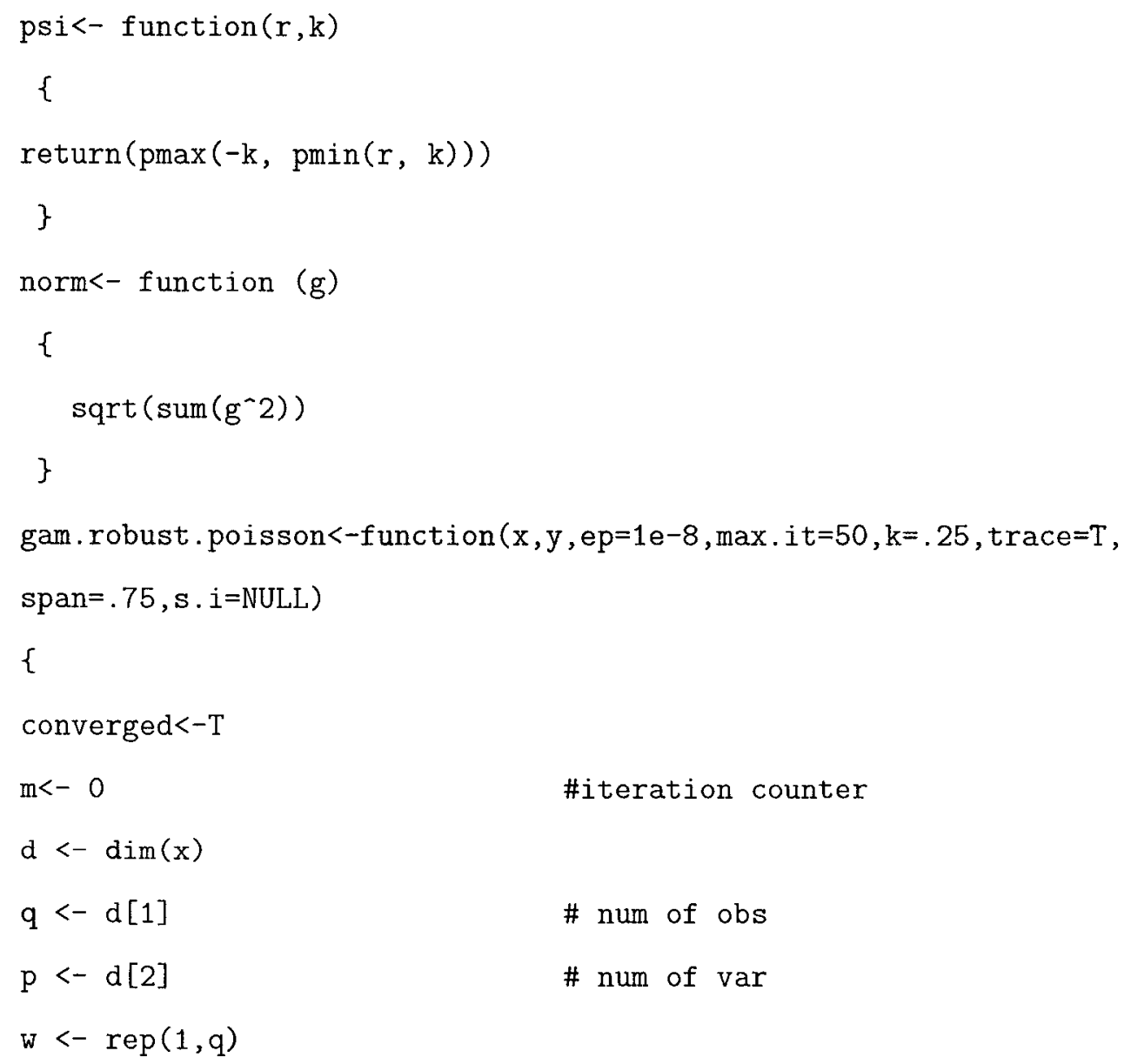




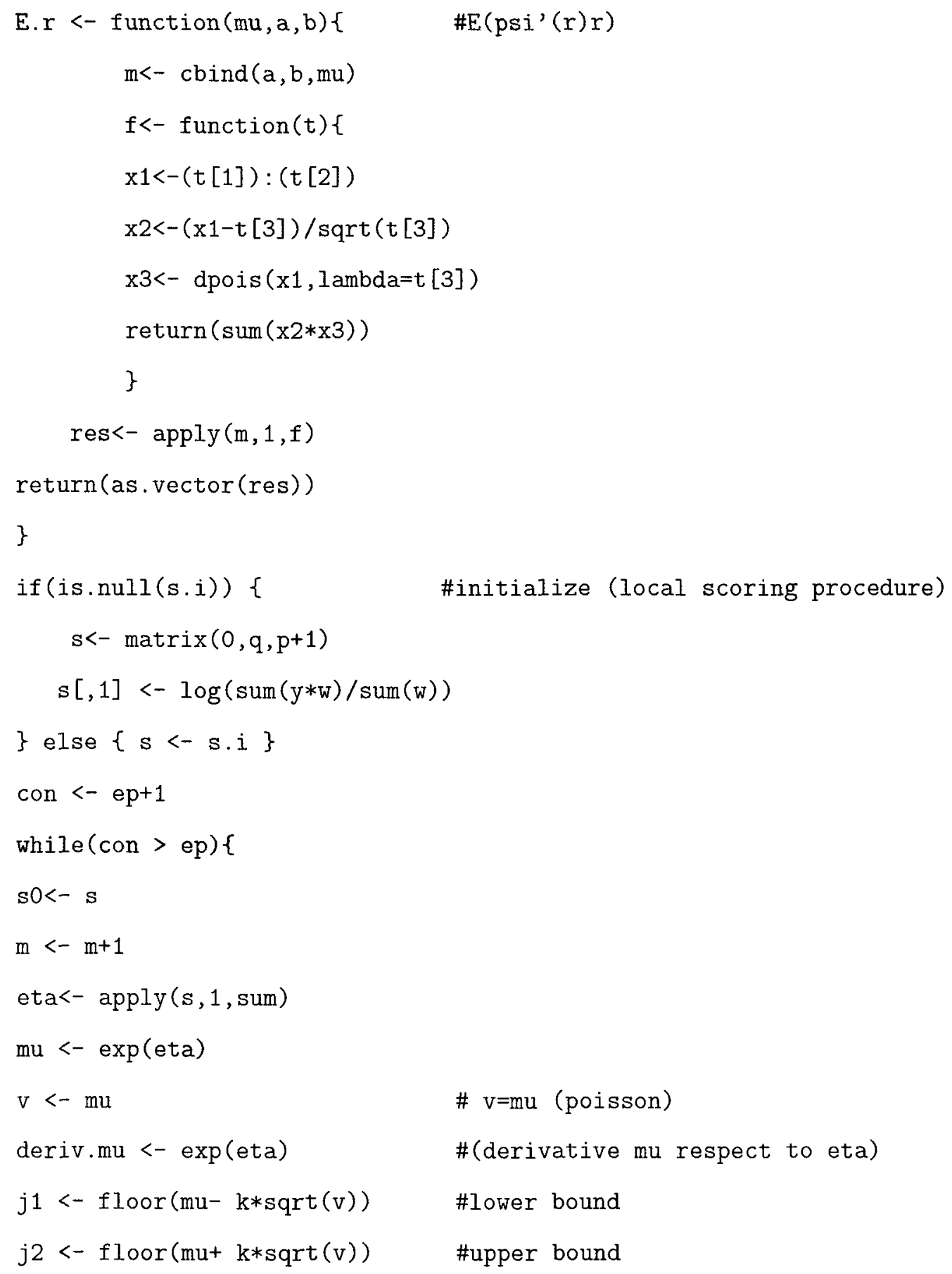




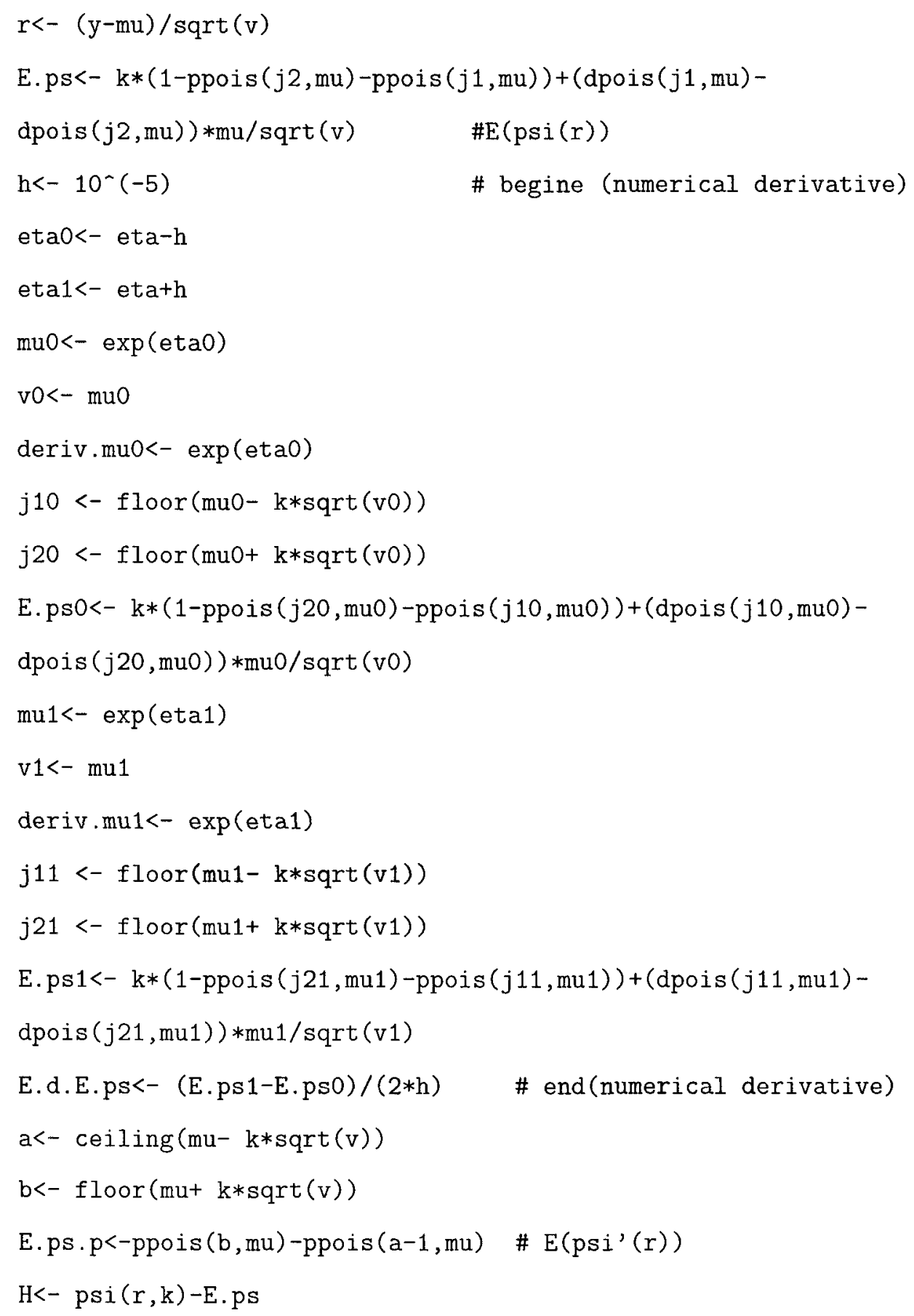




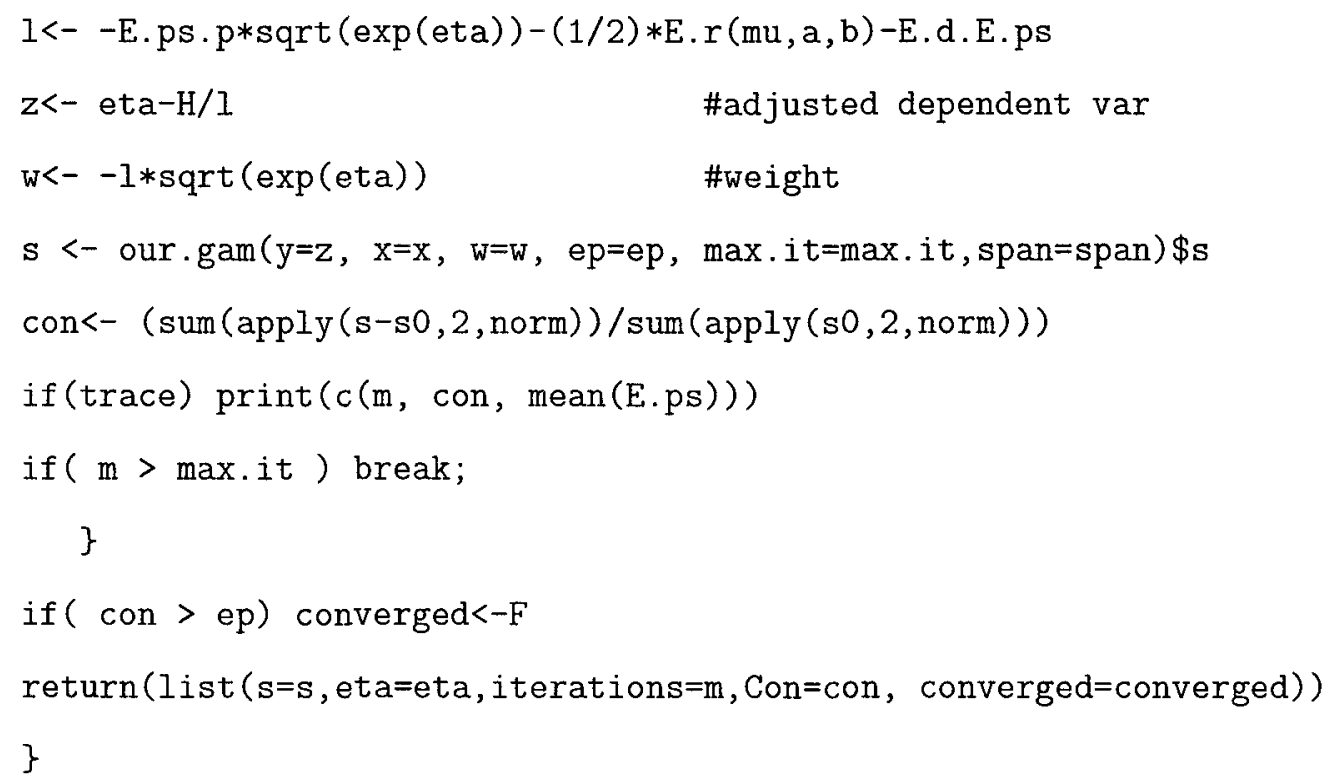




\section{Bibliography}

[1] Bianco, A., Boente, G. (2003) Robust Nonparametric Generalized Regression Estimation. Unpublished manuscript.

[2] Bianco, A. and Yohai, V. (1995). Robust estimation in the logistic regression model. Lecture Notes in Statistics, 109, 17-34. Springer-Verlag, New York.

[3] Cantoni, E. and Ronchetti, E. (2001), Robust Inference for generalized Linear Models. Journal of American Statistical Association, Vol.96, No.455.

[4] Cantoni, E. and Ronchetti, E. (2001). Resistant selection of the smoothing par rameter for smoothing splines. Statistics and Computing, 11, 141-146.

[5] Cleveland, W.S. and Devlin, S.J. (1988). Locally-weighted regression: an approach to regression analysis by local fitting. Journal of the American Statistical Association, Vol. 83, pp 596-610.

[6] Friedman, J.H. and Stuetzle, W. (1981). Projection Pursuit regression. Journal of the American Statistical Association, 76, 817-823.

[7] Hampel, F. (1968). Contribution to the theory of robust estimation. Ph.D. disseration. University of California, Berkeley. 
[8] Hastie, T. Tibshirani, R. Generalized Additive Models. (1986). Statistical Science., Vol. 1, No. 3, 297-318.

[9] Hastie, T. and Tibshirani,R. (1987). Generalized Additive Models.

[10] Huber, P.J. (1964). Robust estimation of a location parameter. The Annals of Mathematical Statistics, 35, 73-101.

[11] Huber, P.J. (1967). The behavior of maximum likelihood estimates under nonstandard conditions. In Proceedings of the Fifth Berkeley Symposium on Mathematical Statistics and Probability. Berkeley, California.

[12] Huber, P.J. (1973). Robust regression: asymptotics, conjectures and Monte Carlo. The Annals of Statistics, 1, 799-821.

[13] Huber, P.J. (1981). Robust Statistics. New York: Wiley.

[14] Künsch, H., Stefanski, L. and Carroll, R. (1989). Conditionally unbiased bounded influence estimation in general regression models with applications to generalized linear models. J. Amer. Statist. Assoc., 84, 460-466.

[15] Lehman, E.L. and Casella, G. Theory of Point Estimation, 2nd Ed. Springer Verlag, New York, (1998) .

[16] McCullagh, P. (1983). Quasi-likelihood function. The Annals of Statistics. Vol.11, No. 1, 59-67.

[17] McCullagh, P. and Nelder, J.A. Generalized Linear Models, 2nd Ed. Chapman and Hall, (1989). 
[18] Neter, J., Kutner, M., Nachtsheim, C. and Wasserman, W. (1996). Appiled Linear Statistical Models, 4th Ed, McGraw Hill, New York.

[19] Plackett, R.L. (1972), Studies in the history of probability and statistics XXIX: The discovery of the method of least squares, Biometrika, 59, 239-251.

[20] Pregibon, D. (1981). Logistic Regression Diagnostics. Ann. Statist., 9, 705-724.

[21] Pregibon, D. (1982). Resistant fits for some commonly used logistic models with medical applications. Biometrics, 38, 485-498.

[22] Rousseeuw, P.J., Leroy, A.M. Robust Regression And Outlier Detection, John Wiley and Sons, Canada, (1987).

[23] Rousseeuw, P.J. (1984). Least median of squares regression. Journal of the American Statistical Association, 79, 871-880.

[24] Rousseeuw, P.J. and Yohai, V.J. (1984). Robust regression by means of Sestimators. In Robust and Nonlinear Time Series. (J. Franke, W. Hardle and D. Martin, eds.). Lecture Notes in Statist., 26 256-272. Berlin: Springer-Verlag.

[25] Rousseeuw, P.J. and van Zomeren, B.C. (1990). Unmasking multivariate outliers and leverage points. Journal of the American Statistical Association, 85, 633-639.

[26] Ronchetti, E. and Staudte, R.G. (1994). A robust version of Mallow's Cp. Journal of the American Statistical Association, 89, 550-559.

[27] Stefanski, L., Carroll, R. and Ruppert, D. (1986). Bounded score functions for generalized linear models. Biometrika, 73, 413-424. 
[28] Stigler, S.M.(1981), Gauss and the invention of least squares, Ann. Stat., 9,465474 .

[29] Wedderburn, R.W.M. (1974). Quasi-likelihood functions, generalized linear models, and the gauss- newton method. Biometrika, 61,3, 439.

[30] Yohai, V.J. (1987). High breakdown-point and high efficiency robust estimates for regression. The Annals of Statistics, 15, 642-656.

[31] Yohai, V.J. and Zamar, R. (1988). High breakdown point estimates of regression by means of the minimization of an efficient scale. Journal of the American Statistical Association, 83, 406-413. 